\title{
Tangible Lighting Controls
}

By

Amardeep Mohanlal Dugar

\author{
A thesis submitted to \\ Victoria University of Wellington \\ in fulfilment of the requirements for the degree of \\ Doctor of Philosophy (Architecture)
}

Victoria University of Wellington

2010 


\section{ACKNOWLEDGEMENTS}

I wish to acknowledge the supervision of Dr. Michael R. Donn, School of Architecture, Victoria University of Wellington, who in his astute, benevolent, candid, methodical and professional manner provided encouragement, freedom, guidance and help to pursue this project and complete it on time. I also gratefully acknowledge the assistance of my secondary supervisor Christopher Cuttle, who from his vast experience and knowledge in the lighting field provided timely advice during the course of this project.

I also gratefully acknowledge the supervision of Professor Werner Osterhaus, Aarhus School of Engineering, and Dr. Aukje Thomassen, Massey University, for their respective roles of primary supervisor and thesis advisor up until the point of their resignation from Victoria University of Wellington.

This project was funded primarily by Education New Zealand and in part by Victoria University. I am thus indebted to both Education New Zealand and Victoria University of Wellington for their financial assistance.

I also acknowledge help given at various times and in different guises by Victoria University staff and students. Dr. Jenny Neale, Dr. Nokuthaba Sibanda, and Lisa Woods for their valuable inputs on survey questionnaire designs and the statistical analysis of data collected. David Harrison for programming the screen-based virtual preset control interfaces. Doug Easterly, Morgan Barnard, and Lee Gibson for their valuable inputs on the use of tactile mobile devices. Gert Verhoog for 
developing the first proof of concept for the web-server. Dr. Stuart Marshall for his patient cooperation in programming the web-server and iPod to be used as a prototype interface. Margaret Maile Petty, Michael Dudding, and the entire post-graduate writing group for continued guidance and support in reviewing my work.

A special mention of thanks to all the participants of the survey research for their invaluable contributions to the project. There are various other individuals who have helped in various ways whom I would to thank: Prof. Dr. Ranee Vedamuthu, Anna University Chennai, for granting permission to conduct experiments at the School of Architecture and Planning; Neill Bryce [Roissy Ltd.], Geoff Maunsell [Concept Lighting], Carey [3-L Ltd.], and Kalaidasan and Chandru [Devi Circuits] for the design and fabrication of the LED colour controllers; Alok Hada [Anusha Technovision Pvt. Ltd.] for supplying the Lutron dimmers; all other electricians, technicians and workmen for developing and fixing various gadgets that were used during the course of this project.

Finally, I would like to thank my family for their immense love, patience and support during the course of this $\mathrm{PhD}$ project. 


\section{ABSTRACT}

'Tangible lighting controls' is used as an umbrella term to describe lighting control systems that are easy to understand and pleasurable to use by end-users. The crucial question posed is, what is the nature of interface designs sought by end-users for maximising interaction with lighting control systems? The manner in which this question is posed implies a fundamental assumption that improved usability and end-user experience are the primary goals.

The concept of end-user understanding of lighting control interfaces is proposed as a basis for improving the usability and end-user experience of lighting control interfaces. Usability engineering methods involving survey research, experimental mock-ups and prototyping have been used to enable end-users to design and evaluate lighting control interfaces. The essential difference is to include end-users' point of view about ease of understanding control functions and pleasure of performing control tasks along with a technical point of view about meeting required standards.

Manufacturers' claims about the effectiveness of existing lighting control interfaces are challenged, and an entirely different way of thinking about interface design is revealed. Such a change of thinking may be seen as a new framework for improved designs of lighting control interfaces as well as evaluation of their usability and end-user experience. 


\section{CONTENTS}

1 Establishing 'Tangible Lighting Controls' .......................................................... 1

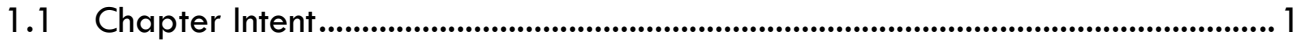

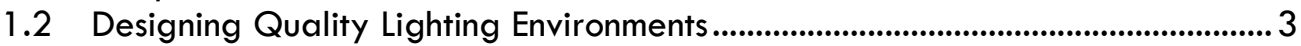

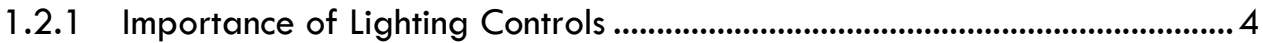

1.2.2 Interaction through Lighting Controls ......................................................................... 5

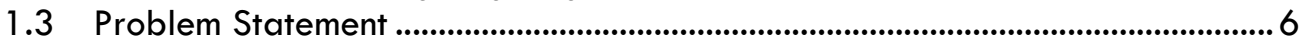

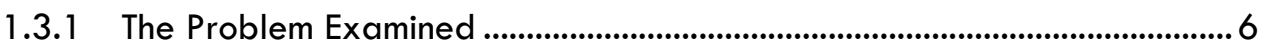

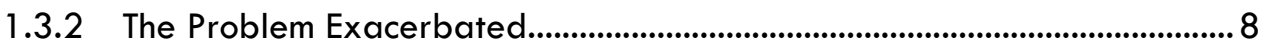

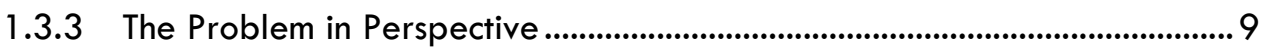

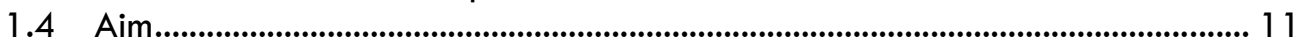

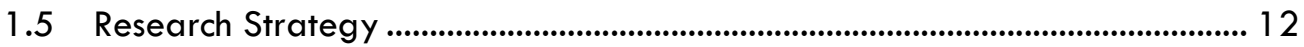

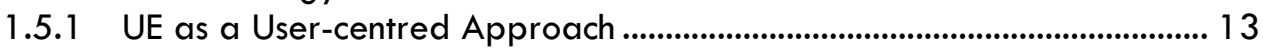

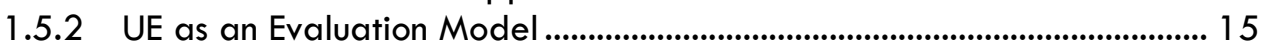

1.5.3 A Hybrid UE Model for Tangible Lighting Controls.................................... 16

1.5.4 Scope and Limitations of the Hybrid UE Model ......................................... 18

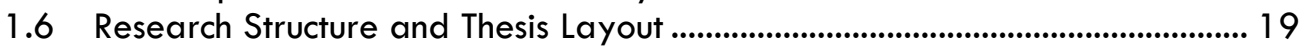

1.6.1 Phase One: Define end-user requirements................................................. 20

1.6.2 Phase Two: Characterise tangible interfaces ............................................. 21

1.6.3 Phase Three: Design interactive studies ....................................................... 21

1.6.4 Phase Four: Evaluate existing interfaces ........................................................ 22

1.6.5 Phase Five: Evaluate virtual prototypes ........................................................... 22

1.6.6 Phase Six: Discuss, review and implement....................................................... 23

1.7 Chapter Inference ............................................................................................................ 23

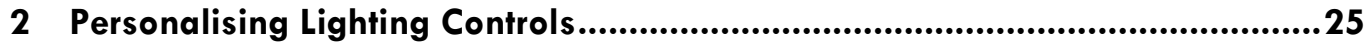

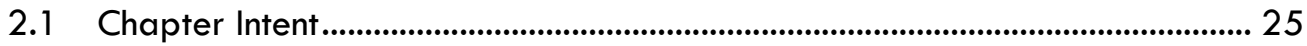

2.2 Lighting Controls: Reintroducing them! .................................................................... 26

2.2.1 Aesthetic Control - Techniques ........................................................................ 28

2.2.2 Aesthetic Control - Strategies ............................................................................ 29

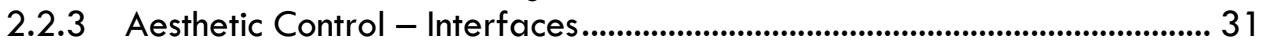

2.3 Personalising Controls: Why is it needed? ............................................................ 32

2.3.1 Tangible Interaction through Personalisation.................................................... 33

2.4 Personalising Controls: A psycho-physiological overview .................................... 35

2.4.1 The Perceptual System ........................................................................................... 36

2.4.2 The Cognitive System .......................................................................................... 37

2.4.2.1 The Cognitive System - Sensory and Short-term memories............... 38

2.4.2.2 The Cognitive System - Attention ............................................................... 39

2.4.2.3 The Cognitive System - Long-term memory............................................ 40

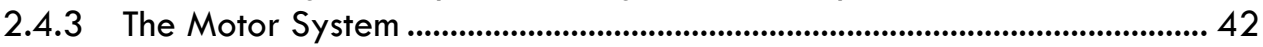

2.5 Personalising Controls: Investigating interfaces................................................... 44

2.6 Chapter Inference ........................................................................................................ 46

3 Understanding End-user Requirements ........................................................51

3.1 Chapter Intent.............................................................................................................. 51

3.2 End-user Recognition .............................................................................................. 53

3.2.1 Recognition Requirements - Hypothesis \# 1 .................................................. 53 
3.2.2 Recognition Requirements - Hypothesis \# 2 2................................................ 55

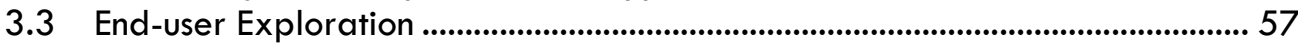

3.3.1 Exploration Requirements - Hypothesis \# 3 ..................................................... 58

3.3.2 Exploration Requirements - Hypothesis \# 4 ..................................................... 58

3.3.3 Exploration Requirements - Hypothesis \# 5 .................................................... 59

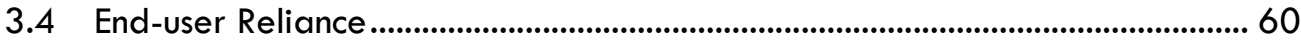

3.4.1 Reliance Requirements - Hypothesis \# 6 .......................................................... 60

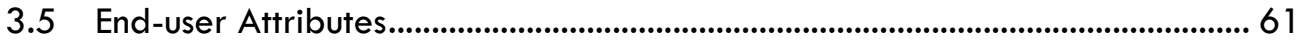

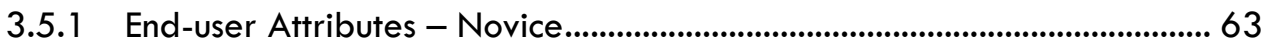

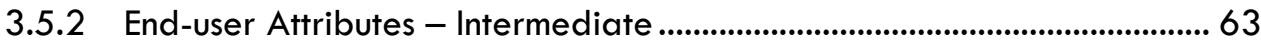

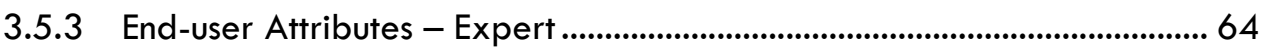

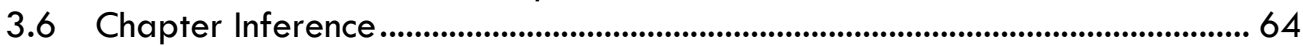

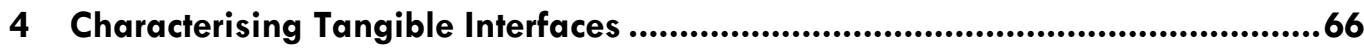

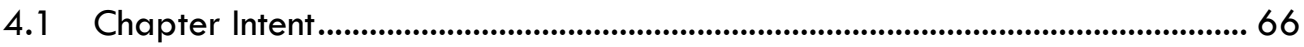

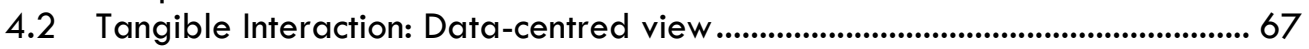

4.2.1 The Principle of Controllable Representations................................................. 69

4.3 Tangible Interaction: Expressive-Movement-centred view ................................. 70

4.3.1 The Principle of Expressive Reciprocations .............................................. 72

4.4 Tangible Interaction: Space-centred view............................................................. 73

4.4.1 The Principle of Contextual Experiences ..................................................... 75

4.5 Tangible Interfaces: Key characteristics ................................................................ 76

4.5.1 Key Characteristic \# 1 - [Rich] Representation ......................................... 76

4.5.2 Key Characteristic \# 2 - [Rich] Reciprocation............................................. 77

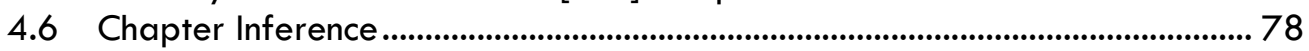

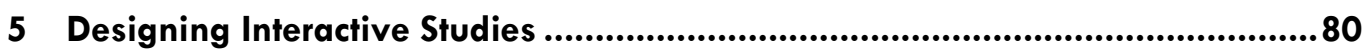

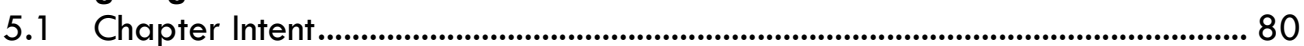

5.2 The Methods: Experimental and Non-experimental............................................ 82

5.2.1 Experimental Method - Quantitative..................................................... 83

5.2.2 Non-experimental Method - Qualitative.................................................. 84

5.3 The Objective: Study end-user behaviour .............................................................. 85

5.3.1 Identify End-users - Sampling population..................................................... 86

5.3.2 Interactive Study \# 1 - Existing interfaces ........................................................ 87

5.3.3 Interactive Study \# 2 - Tangible interfaces..................................................... 88

5.4 The Variables: Independent and Dependent ........................................................ 88

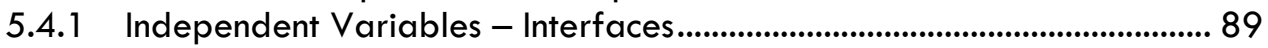

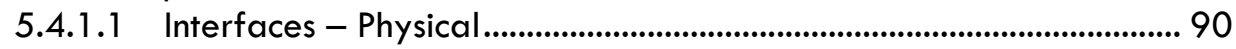

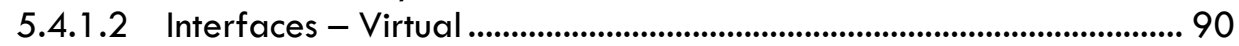

5.4.2 Dependent Variables - Key characteristics............................................... 91

5.4.2.1 Key Characteristic \# 1 - [Rich] Representation .................................... 92

5.4.2.2 Key Characteristic \# 2 - [Rich] Reciprocation....................................... 93

5.4.2.3 Merged Concept - [Ease] Use........................................................................ 94

5.5 The Procedure: Questionnaires.................................................................................... 94

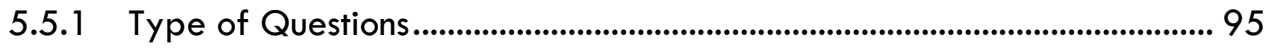

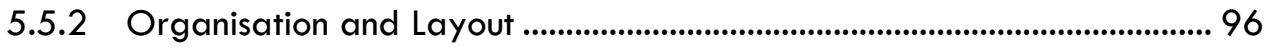

5.5.3 Coding and Analysis System.............................................................................. 97

5.6 The Quality Control Measures: Validity checks................................................... 101

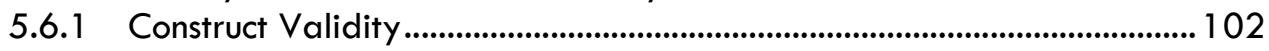

5.6.2 Internal Validity...................................................................................................... 103

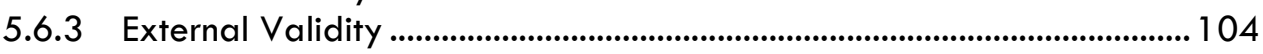

5.7 Chapter Inference ...................................................................................................... 105 


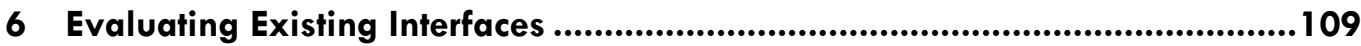

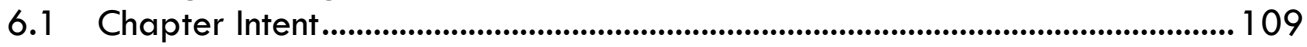

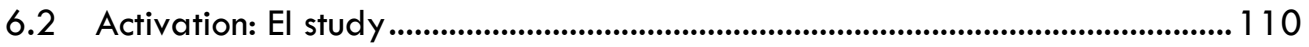

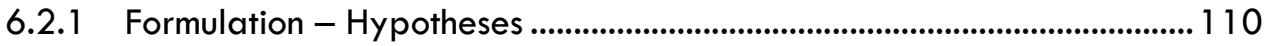

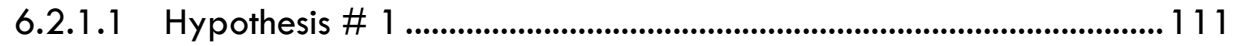

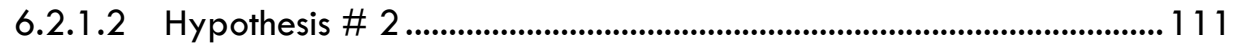

6.2.1.3 Hypothesis \# 3 .............................................................................................. 111

6.2.1.4 Hypothesis \# 4 ............................................................................................ 112

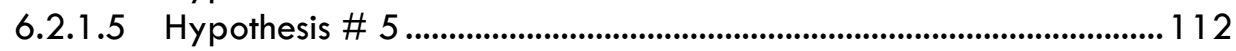

6.2.2 Subdivision - Pilot and Eventual stages..................................................... 112

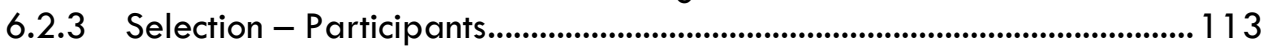

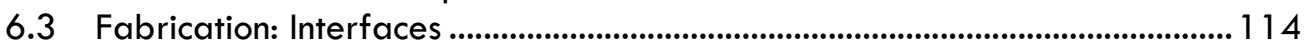

6.3.1 Manual Control Interfaces - Luminous intensity ........................................... 114

6.3.2 Manual Control Interfaces - Luminous colour.............................................. 117

6.3.3 Preset Control Interfaces - Recall scenes ................................................... 119

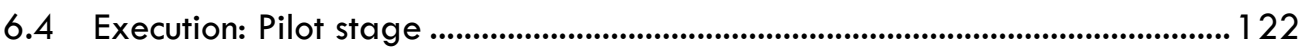

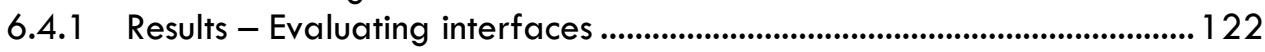

6.4.1.1 Evaluating Interfaces - Appearance................................................... 123

6.4.1.2 Evaluating Interfaces - Accuracy .............................................................. 123

6.4.1.3 Evaluating Interfaces - Learning speed ............................................ 124

6.4.1.4 Evaluating Interfaces - Grabbability ................................................ 124

6.4.1.5 Evaluating Interfaces - Responsiveness ............................................ 125

6.4.1.6 Evaluating Interfaces - Ease of use .................................................. 125

6.4.1.7 Evaluating Interfaces - Summary ................................................... 126

6.4.2 Results - Designing interfaces.............................................................128

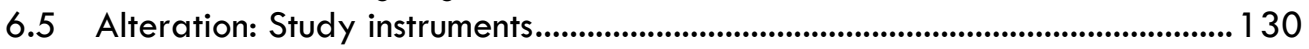

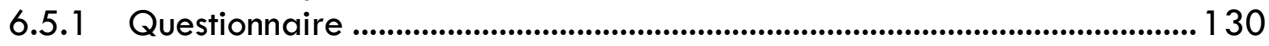

6.5.2 Manual Control Interfaces - Luminous colour................................................ 130

6.6 Execution: Eventual stage ......................................................................................... 132

6.6.1 Results - Evaluating interfaces ....................................................................... 133

6.6.1.1 Evaluating Interfaces - Appearance........................................................ 133

6.6.1.2 Evaluating Interfaces - Accuracy ............................................................. 134

6.6.1.3 Evaluating Interfaces - Learning speed ................................................ 134

6.6.1.4 Evaluating Interfaces - Grabbability .................................................... 135

6.6.1.5 Evaluating Interfaces - Responsiveness .................................................. 135

6.6.1.6 Evaluating Interfaces - Ease of use ........................................................ 135

6.6.1.7 Evaluating Interfaces - Summary............................................................ 136

6.6.2 Results - Designing interfaces....................................................................... 138

6.7 Compilation: El study ............................................................................................. 140

6.7.1 Analysis - Test hypotheses ...................................................................... 142

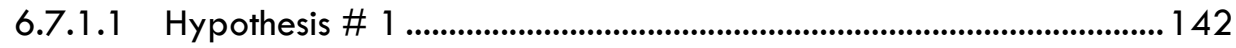

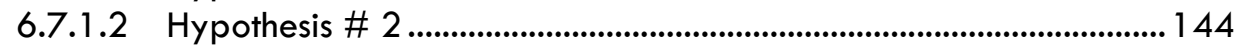

6.7.1.3 Hypothesis \# 3 ........................................................................................ 145

6.7.1.4 Hypothesis \# 4 ............................................................................................. 146

6.7.1.5 Hypothesis \# 5 ....................................................................................... 146

6.7.2 Analysis - Generate new hypotheses ......................................................... 147

6.7.2.1 Hypothesis \# 7 .......................................................................................... 148

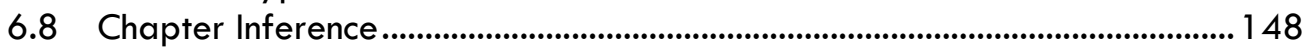

7 Evaluating Virtual Prototypes...........................................................................151

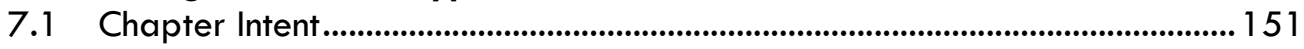

7.2 Activation: Tl study..................................................................................................... 152

7.2.1 Formulation - Hypotheses ............................................................................ 152 


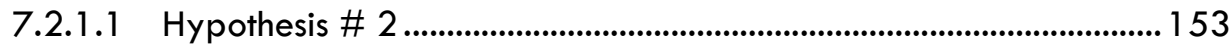

7.2.1.2 Hypothesis \# 5 ................................................................................. 153

7.2.1.3 Hypothesis \# 6 ............................................................................................ 153

7.2.1.4 Hypothesis \# 7 ....................................................................................... 154

7.2.2 Selection - Participants..................................................................................... 154

7.3 Construction: Test environment................................................................................ 154

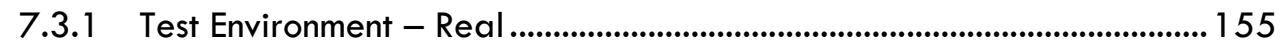

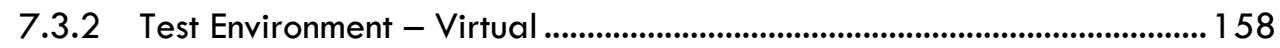

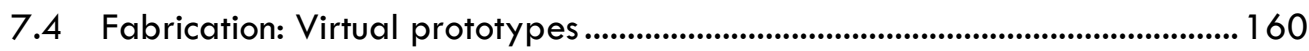

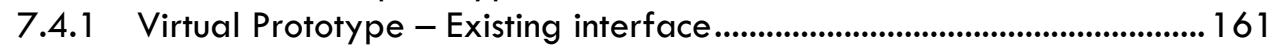

7.4.2 Virtual Prototype - Tangible interface........................................................... 162

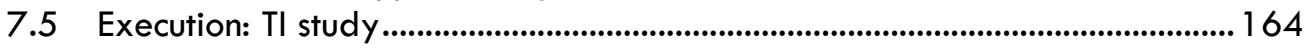

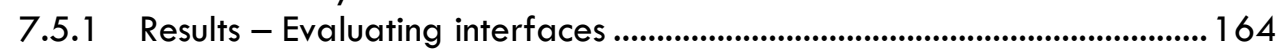

7.5.1.1 Evaluating Interfaces - Appearance...................................................... 165

7.5.1.2 Evaluating Interfaces - Accuracy ........................................................ 165

7.5.1.3 Evaluating Interfaces - Learning speed .............................................. 166

7.5.1.4 Evaluating Interfaces - Responsiveness ............................................ 166

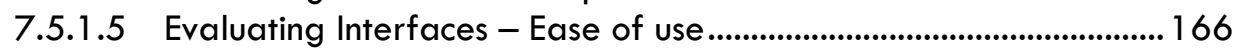

7.5.1.6 Evaluating Interfaces - Summary...................................................... 167

7.5.2 Results - Designing interfaces.................................................................168

7.6 Interpretation: TI study ................................................................................169

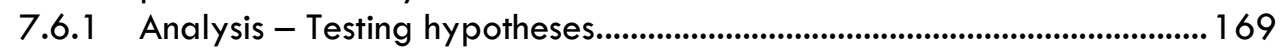

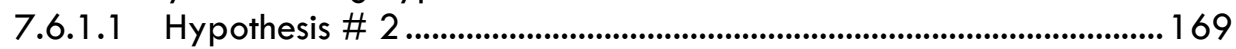

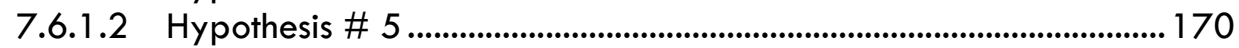

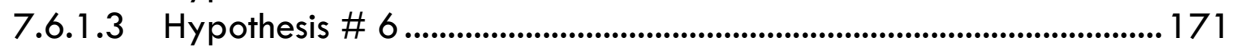

7.6.1.4 Hypothesis \# 7 ....................................................................................... 172

7.7 Chapter Inference .................................................................................................... 172

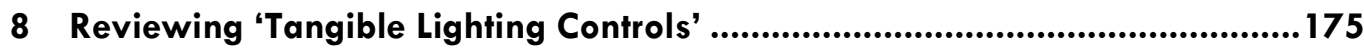

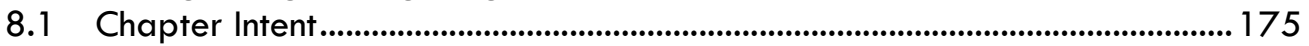

8.2 Research Hypotheses Related Conclusions ............................................................ 175

8.3 Further Conclusions....................................................................................................... 177

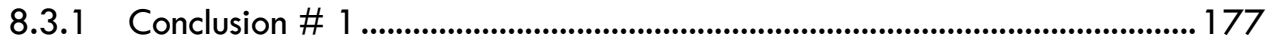

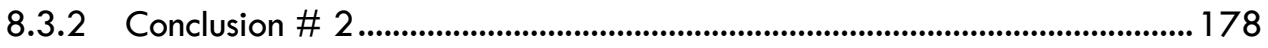

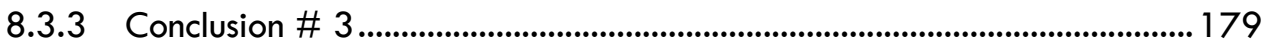

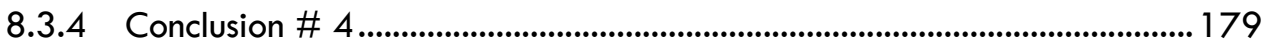

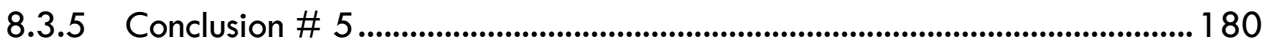

8.3.6 Conclusion \# 6 …............................................................................................ 180

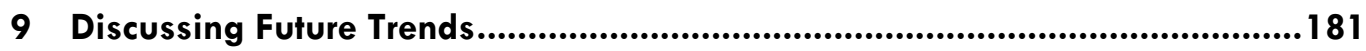

9.1 Directions for Future Work ........................................................................... 181

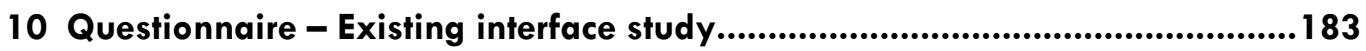

11 Questionnaire - Tangible interface study ....................................................191

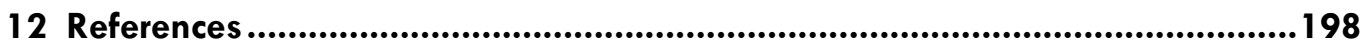




\section{LIST OF FIGURES}

Figure 1 - Aesthetic Control Techniques..

Figure 2 - Aesthetic Control Strategies.

Figure 3 - Preset Control 30

Figure 4 - Relationship between the three human skills, end-user requirements and end-user attributes.

Figure 5 - An example of Grounded Theory coding 100

Figure 6 - Manual control interfaces for luminous intensity .115

Figure 7 - Manual control interfaces for luminous colour [El study: Pilot stage] ......... 118

Figure 8 - The five preset control interfaces. 120

Figure 9 - A split-screen view of the web page with an interface and cursor position selecting the 'Night Light' scene

Figure 10 - A split-screen view of the web page with an interface and cursor position selecting the 'General Meeting' scene.

Figure 11 - Design suggestions for better existing interfaces [El study: Pilot stage] 129

Figure 12 - Design suggestions for future tangible interfaces [El study: Pilot stage] 129

Figure 13 - Manual control interfaces for luminous colour [El study: Eventual stage]

Figure 14 - Design suggestions for future tangible interfaces [El study: Eventual stage]

Figure 15 - Design suggestions for better existing interfaces [El study: Eventual stage]

Figure 16 - Test Environment [Real]

Figure 17 - IR remote control to select luminous colours from Layer - 3, and

SingleFade dimmer to select luminous intensities from Layers -1 and -2 of the

Test Environment [Real].

Figure $18-6 \times 16$ scenes of the test environment......

Figure 19 - Test environment [Virtual] 158

Figure 20 - A participant using the interface in the immersive virtual test environment

Figure 21 - Virtual Prototype [Existing] 161

Figure 22 - Virtual Prototype [Tangible] 


\section{LIST OF TABLES}

Table 1 - Pros and cons of existing interfaces

Table 2 - Pros and cons of existing interfaces [contd.].

Table 3 - [El study: Pilot stage] Summary of Means, Standard Deviations and Minimum-Maximum range for the different luminous intensity and colour control interfaces

Table 4 - [El study: Pilot stage] Percentages of responses and mean ranks for the different luminous intensity and colour control interfaces

Table 5 - [El study: Pilot stage] Summary of Means, Standard Deviations and Minimum-Maximum range for the different preset scene control interfaces..... 128

Table 6 - [El study: Pilot stage] Mean ranks for the different preset scene control interfaces

Table 7 - [El study: Pilot stage] Summary of participants' suggestions for most desirable interface designs.

Table 8 - Summary of participant demographics from India and New Zealand [EI study]

Table 9 - Summary of Means, Standard Deviations and Minimum-Maximum range for the different luminous intensity and colour control interfaces [El study: Eventual stage]

Table 10 - Percentages of responses and mean ranks for the different luminous intensity and colour control interfaces [El study: Eventual stage].

Table 11 - Summary of Means, Standard Deviations and Minimum-Maximum range for the different preset scene control interfaces [El study: Eventual stage]....... 138

Table 12 - Mean ranks for the different preset scene control interfaces [El study: Eventual stage]

Table 13 - Summary of participants' suggestions for most desirable interface designs [El study: Eventual stage]. 140

Table 14 - Analysis of participant preferences from the existing interfaces. 141

Table 15 - Summary of participant demographics [TI study] 164

Table 16 - Summary of Means, Standard Deviations and Minimum-Maximum range for the existing and tangible interface prototypes [TI study]

Table 17 - Mean ranks for the existing and tangible interface prototypes [TI study] 
Table 18 - Summary of participant comments and suggestions [TI study]...................168

Table 19 - Summary of participant suggestions for better existing interfaces from

Existing interface study ........................................................................................................ 178 


\section{CHAPTER ONE}

\section{Establishing 'Tangible Lighting Controls'}

\subsection{Chapter Intent}

Chapter 1 establishes this $\mathrm{PhD}$ project. The principal aim of this $\mathrm{PhD}$ project is to establish the concept of tangibility in lighting control systems. Tangible lighting controls are easier to understand and more pleasurable to use by end-users. Lighting controls are considered "the next frontier" in lighting research: their benefits include the hard-to-quantify benefits of end-user satisfaction and productivity, and the more easily quantified benefits of energy efficiency (Maniccia, Rutledge, Rea, \& Morrow, 1999, p. 42). Research however shows that despite these benefits end-users have difficulty in understanding and consistently using existing lighting control systems (Escuyer \& Fontoynont, 2001; Moore, Carter, \& Slater, 2002a, 2002b, 2004). Bordass, Leaman, and Bunn argue that end-users like to work with technologies that enable creative participation in solutions without feeling alienated or looking silly (2007b, p. 8). Existing lighting control systems have a 'display-only' function such as numerical or graphical displays on screens, and do not offer or provide end-users with a level of interactive control on the input side, such that creativity is fostered. Gehnen further argues that operation of lighting controls not only has to be plainly understandable; it also has to be fun $(2008$, p. 19). This implies that for lighting control systems to be 'wholeheartedly' accepted by end-users, values beyond system functionality need to be taken into consideration, such as creativity and playfulness. 
This $\mathrm{PhD}$ project argues that tangible interaction can provide a means to integrate two aspects of interactive lighting control: ease of understanding control functions and pleasure of performing control tasks. Tangible user interfaces [TUI] and tangible interaction are terms used in the realm of Computer Science and Human-Computer Interaction $[\mathrm{HCl}]$ where tangibility is viewed as a means of making virtual information in the computer subject to physical manipulation outside the screen (Hornecker \& Buur, 2006; Ullmer \& Ishii, 2001). Jensen, Buur, and Djajadiningrat however, argue that tangible interaction is not restricted to controlling digital data and includes tangible appliances or remote control of the real world (2005, p. 9). Although lighting controls are not used as information processing devices like computers, information about controlling and monitoring the lit environment has to be richly represented on interfaces and remote controls for performing control tasks that match end-user skills. Ross and Keyson provide a different perspective to tangible interaction by arguing that the total experience of fulfilling a task should be taken into account while considering interactive systems, where social, personal, and emotional engagement as well as expression are salient factors in interaction, along with ease of operation and efficiency (2007, p. 69). Interestingly, Overbeeke, Diajadiningrat, Hummels, and Wensveen describe TUls as having the potential for creating richer interaction experiences, incorporating emotional expression in tangible interaction (2002). Therefore, by focussing on TUls that offer personal expression, the physical interaction possibilities of lighting control interfaces can be designed such that they offer end-users freedom to express emotion alongside functional information.

The overall understanding of the reasons for undertaking this project requires a general overview of the project. Section 1.2 describes the general background to the project, while Section 1.3 offers a brief 
description of the problem under investigation. Section 1.4 provides a statement of the aim of this project, before Section 1.5 describes the research strategy employed for achieving these aims. Section 1.6 describes the research structure and thesis layout in the form of the content for each subsequent chapter. Section 1.7 then provides the summary and inferences of Chapter 1.

\subsection{Designing Quality Lighting Environments}

My foremost goal as a practicing architectural lighting designer is to design quality lighting environments. Lam argues that "all visual design is de facto lighting design" and design of quality environments involves the "design of human sensory experiences" (1977, p. 13). Winchip however argues for a more focussed viewpoint that design of quality lighting environments requires an insight into the "interaction between a person and the environment" to unravel how illumination can affect people, thereby optimising the "person-in-environment" system (2005, p. 318). In the realm of interaction design, human sensory experiences are created by a phenomenon called tangible interaction where people bodily interact with everyday material objects in the environment (Diajadiningrat, Overbeeke, \& Wensveen, 2002, p. 288; Hornecker, $2006 a$, p. 21 ). Therefore tangible interaction with lighting may be used as a means of creating human sensory experiences in lit environments.

Howlett argues that because light is an intangible medium, its inherent intangibility can only be redressed through the physical interface provided by lighting controls (2001, p. 94). This implies that the physical interface provided by lighting controls may be used as a means of achieving tangible interaction and rich sensory experiences with the 
lighting, which in turn might lead to the design of quality lighting environments.

\subsubsection{Importance of Lighting Controls}

Lighting controls are considered an integral part of any lighting design scheme. Chapter 27 of the IESNA Lighting Handbook states that lighting controls in buildings are installed to provide end-users with "aesthetic and energy management control" over the electric lighting system (Rea, 2000, p. 1). In my own experience, I have observed that lighting controls provide remarkable flexibility to accommodate a variety of activities in the same space as the lighting can be adjusted to create the required mood and ambience. A working conference room can be transformed into an evening dining area by a lighting system with properly designed controls. Winchip states that general, task, accent, and decorative light sources that have the provision of controls can enhance the purpose of the light source (2005, p. 239).

Moore, Carter, and Slater argue that this opportunity of providing lighting systems that enhance end-user satisfaction with their local conditions is surely an attractive option for designers and their clients, particularly when coupled with energy savings (2004, p. 145). Although the ability of controls to improve morale and productivity in a directly measurable sense may be questioned, Miller argues that allowing endusers a measure of control once the lighting is in place can have positive returns in morale and productivity $(2001$, p. 4). This being the case, two questions raised by Aston remain unanswered: first, why end-users prefer local controls for lighting and second, what methods can be used to provide local controls $(2004$, p. 145). Both these questions are looked at in more detail in Chapter 2. 


\subsubsection{Interaction through Lighting Controls}

Lighting controls play a critical role in inducing end-user interaction with electric lighting systems. This interaction is brought about by functions of turning the lights on/off using a switch and adjusting light output up/down using a dimmer (Dilovie). Lam argues that our most comfortable and pleasant spaces are those in which end-users retain control over the layout and fine tuning of the lighting (1977, p. 12). A review of end-users' evaluation of office buildings concludes that the mere perception of having control leads to increased end-user satisfaction (Leaman, 1995, p. 13). Moore, Carter, and Slater reinforce this by reporting that end-users prefer the ability to choose conditions in office environments rather than being forced to accept conditions chosen for them, even if these are objectively better (2004, p. 142). As simply put by Egan and Olgyay, people want to have localised control of their work environment; end-users often turn on switches when entering a space regardless of visual need (2002, p. 204).

A study of end-user attitudes towards user-controlled lighting in offices carried out by Moore, Carter, and Slater provides evidence that endusers place a high level of importance on being able to control electric lighting; certain end-users pro-actively use controls to set preferred conditions and not solely use controls in response to discomfort (2002b, pp. 211,215$)$. This may be a reason why lighting in private residences is generally considered pleasing as it is usually designed and can be adjusted to suit user-specific needs for visual information, and not to achieve some mandatory prescribed light levels, as described by Lam (1977, p. 12). Jennings, Rubinstein, DiBartolomeo, and Blanc further report that the shortage of well-monitored installations showing the sustained benefits of different lighting control strategies is probably a 
contributor to the relatively slow adoption of lighting controls in nonresidential buildings (2000, p. 1).

\subsection{Problem Statement}

While in theory there is a general agreement about the benefits of sophisticated controls for electric lighting, practice demonstrates difficulty in embracing and consistently using them. Winchip explains that sometimes an expensive, well-planned lighting control system may be turned off, or end-users do not know how to use it (2005, p. 239). Bordass, Leaman, and Bunn reinforce this by arguing that these control systems "challenge rather than assist, and confuse rather than inform" (2007b, p. 8). In one of my own designs for a private residence, a sophisticated and well-engineered lighting control system was rendered redundant and under-utilised merely due to the fact that my client had difficulty in understanding its operational use. Norman quite rightly describes this situation: "but what good is technology if it is too complex to use" (1990 c1988, p. 30).

\subsubsection{The Problem Examined}

The problem is well supported by the finding made by Moore, Carter, and Slater that dissatisfaction, where it occurs, arises as a result of controls perceived as being unusable (2004, p. 142). Additionally, an earlier study carried out by Moore, Carter, and Slater reveals that endusers use controls in the way they find easiest, but not necessarily in the manner intended, or technologically desirable (2002a, p. 199). Preece, Rogers, and Sharp argue that end-user interaction with products requires goals of bringing "Usability and End-user Experience" in the design process (2002, pp. 13-14). Usability goals in the straightforward sense 
are a self-evident requirement for all technical equipment and are concerned with meeting specific usability criteria, while end-user experience goals are largely concerned with explicating the quality of end-users' experience with designs in terms of fun and pleasure of use. These goals differ in terms of how they can be met and through what means.

Usability in the context of this thesis research is regarded as ensuring that end-users can learn to use the lighting control systems quickly and effectively. This involves optimising end-users interactions with a straightforward "operational" usability, allowing the functionality of the systems to be discovered and used through factors from the "constructivist and instructivist" approaches described by Mayes and Fowler (1999, p. 486). Constructivist approaches focus on factors supporting the end-user in the performance of tasks, which are designed for active problem solving and manipulation. Instructivist approaches on the other hand involve factors emphasising the impact of content presentation on the end-user like accessibility, vividness, power of explaining control functions, and appropriateness of its representation. End-user experience differs from the more objective usability aspect, in that they are concerned with how end-users experience the interactive lighting from their perspective, rather than assessing how useful the lighting control system is from its own perspective. This involves explicating the nature of the end-user experience about how the interaction with the lighting 'feels' in subjective terms of fun and pleasure of use. This implies that the interaction between end-users and lighting control systems, and the failure to deal with their usability and end-user experience in a coherent fashion, emerge as the key issues. These factors seem most responsible for the present under-utilisation and lack of understanding of lighting control systems. 


\subsubsection{The Problem Exacerbated}

The complexity of modern control interfaces is cited as one of the reasons for the reduced acceptability and usability of lighting control systems. This is based on an observation made by Escuyer and Fontoynont in their field study of office workers' reaction to lighting controls (2001, pp. 8889). Recent advances in technology have introduced several digital interfaces like control panels and remote controls, which manufacturers claim can empower end-users to access-and-control lighting systems in 'novel ways.' According to a local distributor for a leading lighting controls manufacturer in India, their latest pushbutton dimming interface with its ability to accurately select desired illumination levels will revolutionise the market. The distributor has made further claims that as a result of this 'revolutionary' design, they are considering a total phaseout of their inaccurate and 'old-fashioned' rotary dimmers. Maeda however argues that "the endless array of buttons" on pushbutton remote controls for modern electronic equipment can be "notoriously confusing" (2006, p. 5).

Existing lighting control interfaces that require end-users to interact with them to select their desired lighting scenarios have not necessarily been designed with end-users in mind. Tidwell argues that due to the high expectations of end-users if the interface is not easy to use "out of the box," they will not think well of it $(2005$, p. xi). End-users' preferred workflow is often misunderstood while designing these interfaces, or the use of vocabulary is wrong, or it is just made too hard for them to figure out what the interface is meant to do, even though the interface obeys all the standards. Escuyer and Fontoynont's study reports that although the given remote control meets all standards of allowing selection and storage of lighting scenes, no respondent was able to store personal lighting scenarios, or knew this was possible (2001, pp. 80, 88). The 
above study also shows that these interfaces have been engineered as control systems to effectively perform a set of lighting control functions, often neglecting the fact that real people will use them with little or no previous knowledge on using them.

\subsubsection{The Problem in Perspective}

This thesis research hypothesises that existing designs of lighting control interfaces can be improved to meet end-user requirements for usability and end-user experience. Architectural lighting designers create different lighting scenarios with interactive user-controlled electric lighting systems to offer end-users rich sensory experiences. Therefore, the physical design and visual representations of lighting control interfaces should convey rich information about the interactive qualities of the lighting control system for end-users to appropriately use them and derive pleasure while using them. Existing designs of lighting control interfaces either convey partial or no information at all about the different lighting scenarios they are intended to create and how endusers can use them. In Diajadiningrat, Overbeeke, and Wensveen's opinion, the essence of usability of interfaces lies in first communicating the purpose of the action, and second in strengthening the coupling between the action and the feedback (2002, p. 285). This informs endusers about what the result of their actions will be as well as the action that is carried out while showing that the interface is responding. Therefore the conjecture of this thesis research is: interfaces that neither communicate the purpose of the intended control functions, nor provide appropriately coupled feedback of the resultant lighting effects are responsible for end-users taking few initiatives in understanding the control functions. This in turn undermines and under-exploits the lighting control systems' true benefits, and hinders end-user interaction with the lit environment. 
This thesis research explores the proposition that TUls can be a mode of responding to the "implied challenge of producing lighting systems" with which end-users have "a desire to interact," as indicated by Howlett (2001, p. 94). Ullmer and Ishii coined the term "tangible user interface" to describe the abacus as one of TUl's "compelling prototypical example" (2001, p. 579). The abacus beads, rods, and frame simultaneously serve as manipulable physical representations of numerical values and operations, and as physical controls for directly manipulating their underlying associations. The feedback provided by the abacus is immediate and coupled with the numerical operations. Although the direct application of this example in terms of physicality of representation and controls is not taken into consideration for this thesis research, lighting control interfaces should have a seamless integration of the representations of lighting control functions with their subsequent control in order to provide coupled feedback. This implies that a tangible lighting control interface like the abacus might offer the mediating control for end-user interaction with the lit space.

This thesis research also intends to understand end-users' understanding of lighting control interfaces. As new designs of interfaces emerge, their descriptions should include end-users' evaluation and understanding of usability and end-user experience. This is based on the concept of "second-order understanding" developed by Krippendorff and Butter (2008, p. 354). Second-order understanding requires listening to what end-users say they experience with lighting control interfaces, and acknowledging their understanding as legitimate, not inferior or mistaken, even when it deviates significantly from the interface designer's intent. Unfortunately little published work is available that reports end-users' evaluation of these new interfaces or documents their needs. Formal end-user evaluations are needed to demonstrate the usability and end- 
user experience of these interfaces: to validate not only that the interface can efficiently and accurately represent lighting control functions, but that end-users can perform the necessary actions with the corresponding new interfaces to efficiently and accurately select their desired lighting scenarios.

\subsection{Aim}

This thesis research aims to promote end-user interaction with lit environments through tangible lighting control interfaces. The crux lies in exploring how TUls can work well for end-users in interacting with the lit environment. Equally, there is need for an approach supporting research dealing with the real needs of end-users for the control of lighting; most in situ studies are concerned only with energy savings, as noted by Escuyer and Fontoynont (2001, p. 77). However, as end-users do not always have the language or introspective skills to explain what interface characteristics they really need to accomplish their desired lighting scenarios, it is important to determine useful tools that can enable end-users to state their needs. Therefore this thesis research aims firstly, to explore design principles and particular characteristics of tangible lighting control interfaces and secondly, to develop a methodology for end-user evaluation of lighting control interfaces.

Design principles and key characteristics of tangible lighting control interfaces based on end-user requirements are proposed in this thesis research, which are then used to explore the design of a prototype tangible lighting control interface. Djajadiningrat, Wensveen, Frens, and Overbeeke argue that the only way of exploring and evaluating the "beauty of interaction with things" is by making "experiential prototypes" 
(2004, p. 297). These characteristics are also used as evaluation tools that will enable end-users to evaluate the usability and end-user experience of lighting control interfaces. The results are intended to suggest corrective measures to existing interfaces and/or provide ideas for future tangible interfaces.

\subsection{Research Strategy}

The research strategy used in this study for designing tangible lighting control interfaces and developing tools for end-user evaluation of lighting control interfaces is a hybrid derived from a discipline called Usability Engineering [UE]. UE is a process of writing down formal, verifiable, and hence measureable usability criteria that has emerged over many years in interaction design with various proponents. Preece, Rogers, and Sharp argue that focus on end-users, specific usability and end-user experience criteria, and iteration are the three characteristics that have an integral role in designing interfaces (2002, p. 170). Krippendorff and Butter reinforce this by arguing that design focussed on end-users acknowledges their role in actively constructing designs conceptually, linguistically, and materially - being concerned with them, handling them, and putting them to work (2008, p. 354). However, a user-centred approach for design that observes end-user behaviour with lighting control interfaces and enables them to provide feedback can be biased in subtle ways. Therefore, using formal and quantitative scientific methods of observation and documentation are considered more valuable to attain knowledge about end-users. Tidwell argues that a scientific approach helps us see the world as it actually is, and not how we think it is $(2005$, p. 7). Cozby further underlines the advantage of using a scientific approach over other ways of knowing about the world 
as it provides an objective set of rules for gathering, evaluating, and reporting information, such that ideas can either be refuted, or supported and replicated by other researchers (2001, p. 5).

UE represents such scientific methods as it provides systematic methods and tools for the complex task of designing interfaces in a reliable and replicable manner (Butler, 1996, p. 59). Preece, Rogers, and Sharp explain that UE involves specifying quantifiable measures of product performance, documenting them in a usability specification, and assessing the product against them $(2002$, p. 182). This approach can be used to make changes to subsequent versions of a product based on end-user feedback from carefully documented results of tests for the earlier version. Butler argues that the objective of prototyping in $U E$ is to implement the prototype interface as the first "best-try" at the final version, and then improve it in a systematic refine-and-test cycle (1996, p. 68).

\subsubsection{UE as a User-centred Approach}

This thesis research requires a user-centred approach for designing tangible lighting control interfaces. Preece, Rogers, and Sharp argue that when end-users and designers engage with the domain and discuss requirements, different insights into what is needed, what will help in their better understanding of technology, and what is feasible to attain will emerge (2002, p. 170). Butler reinforces this by reporting that a usercentred approach begins with critical mapping between end-users' needs and the application of the technology (1996, p. 64). The process used to refine designs based on end-user evaluation and feedback is called iteration, where different activities with the domain inform each other and can be repeated. Butler further reports that iteration is a key principle in UE, where the cycle of evaluation continues until it yields 
satisfactory results (1996, p. 60). Therefore the original design can be refined repeatedly based on the results of the evaluation. There are two ways of providing end-users with opportunities for evaluation and feedback namely, the conventional $\mathrm{HCl}$-based approach and the participatory design approach.

Rubinstein and Hersh state that the conventional $\mathrm{HCl}$-based 'user-centred' approach places end-users in a role of reaction for providing feedback to ensure that their needs are met with the given interface design (1984). However, as Muller, Wildman, and White have pointed, this form of feedback is exclusively based on reaction rather than initiation (1993, p. 65). A further problem with this kind of "asymmetrical relationship" as pointed by Scaife, Rogers, Aldrich, and Davies is that the onus is entirely on the designer to take on board and translate end-user reaction, with the actual contribution made by end-users for the redesign of interfaces being "too little, too late" (1997, p. 343). Schuler and Namioka provide an antithesis to the 'conventional user-centred' approach called the 'participatory design' approach, where end-users are encouraged to provide feedback by giving them a more equal and responsible role as partners and co-peers in developing systems that fit their needs (1993, p. $\mathbf{x}$ ). This approach is effective for projects where end-users and designers view each other as peers, requiring an intense level of initiated participation from both parties. However, as Muller, Wildman, and White have pointed, the major failing of this approach is that it often encourages a premature focus on detailed design, without supporting a critical participatory analysis of higher level issues, such as task flow and user-actions (1993, p. 66). 


\subsubsection{UE as an Evaluation Model}

This thesis research requires a model to develop tools for evaluating lighting control interfaces. Cozby insists on the use of empirical tests for scientific observation and evaluation (2001, p. 5). Butler has identified two principal types of evaluation that have emerged in UE: first is empirical user testing, and second is usability inspection methods (1996, pp. 69-71). In empirical user testing, data from end-users' interactions with designs during performance of task scenarios is recorded with measures that generally address "learnability, throughput, and user satisfaction" (p. 69). Both objective and subjective data can be gathered in empirical user testing. The objective data can be analysed using statistical procedures such as ANOVA to "measure the designs' usability against objective criteria and assess its suitability to support the intended task improvements" (p. 70). The subjective data from test participants are also very informative for "checking satisfaction and diagnosing problems" (p. 70).

Nielsen and Mack's popular usability inspection method is called Heuristic evaluation $[\mathrm{HE}]$, which uses a small team of experts who independently inspect the interface design (c1994, p. 26). Butler argues that rather than checking for conformance to inflexible standards, expert inspectors in HE search for ways in which "principles of good design have been applied or contradicted" in the interface design (1996, p. 71). The goal of $\mathrm{HE}$ is to find major usability problems in the interface design without using the large set of resources typically required for usability testing. Therefore the technique is a discount approach to usability by using simple and approximate methods, instead of the more formal and exact methods of testing. During evaluation, the expert inspector goes through the interface design several times and compares them with a list of recognised usability principles. Nielsen and Mack argue that the expert 
inspector is also allowed to consider any additional usability principles or results that come to mind that may be relevant to the design (c1994, p. 28). Muller, Matheson, Page, and Gallup have further derived a participatory inspection technique from HE called Participatory heuristic evaluation [PHE], which adds end-users to the list of expert inspectors (1998, p. 13). Therefore the end-users join the inspection team as workdomain expert inspectors, complementing the more abstract knowledge of the traditional HE expert inspectors.

\subsubsection{A Hybrid UE Model for Tangible Lighting Controls}

Design of lighting control interfaces requires principles of good design along with end-user participation, while evaluation of these interfaces requires empirical testing with end-user reactions. I have therefore adopted an approach that improves upon the two extremes of the usercentred approaches and hybridises them with the two evaluation models. The approach begins with the literature review of specific lighting control techniques, strategies and interfaces that may enable end-users to experience 'sensory richness' in lit environments. This data is used to cognitively analyse and itemise those requirements of end-users that can maximise usability and end-user experience of lighting control interfaces and thereby achieve tangible interaction. These itemised requirements are formulated into hypothetical predictions about end-user behaviour with lighting control interfaces for empirical testing. Similarly, literature reviews of the most dominant views on tangible interaction are used to evolve principles for designing tangible lighting control interfaces. These principles form the basis for developing key characteristics of tangible lighting control interfaces, which are then transformed into evaluation tools with quantifiable measures of usability and end-user experience. 
The approach adopts the attribute of empirical tests where test subjects can react to the usability and end-user experiences of existing lighting control interfaces as well as participate in the design of their most desirable interfaces. The idea is systematically to engage end-users in the lighting control process while enabling them to state their opinions about existing interfaces, as well as needs, hopes and aspirations about their desired interfaces. The act of giving different choices of existing interfaces encourages end-users to express themselves at greater length about their personal use of these interfaces, as documented by Escuyer and Fontoynont (2001, p. 78). Drawing insights from anthropology, behavioural psychology, distributed cognition and phenomenology, test sequences performed by subjects with existing interfaces are first analysed. From the analysis of the characteristics of end-user forms of practice with existing interfaces, the move is towards designing tangible interfaces.

Finally, inputs from these preliminary sessions are combined with the principles for designing tangible lighting control interfaces to evolve a prototype tangible interface, which is subsequently tested and evaluated by end-users using a similar procedure of reaction and participation, and their design ideas further iterated. The intent is to find qualities so general that they can provide feedback for bringing corrective measures to the designs of existing interfaces, as well as for the designs of future tangible user interfaces in general. Inspiration for this approach is drawn from Jensen, Buur and Djajadiningrat's ways of extracting qualities of actions from a wide range of human actions, with the aim to find and discuss the finer details of actions, and to preserve those qualities when using them as a basis and inspiration for new interface designs (2005, p. 10). 


\subsubsection{Scope and Limitations of the Hybrid UE Model}

Clearly, there is much to be gained from adopting this hybrid approach that brings out the best from each of the individual approaches and models. On an individual basis, the problems faced by end-users with existing interfaces can be documented using the conventional approach while end-user ideas for better interface designs can be documented using the participatory design approach. However, both these kinds of inputs can be documented simultaneously using the hybrid model. Similarly the use of empirical user testing and heuristic evaluation, results in two simultaneous activities: firstly, the identification of problems faced by end-users with interface designs; and secondly, the development of principles for designing tangible lighting control interfaces. Based on end-user experiences, insights into which interface methods are effective and what motivates them to interact with the lighting can be listed using this approach. Therefore this thesis research is informed by the intended use of the interface, target domain, and relevant practical considerations throughout the project. More importantly, like all scientific approaches the hybrid approach can be observed, evaluated, and replicated by other researchers. Thus in contrast to intuition or authority, this approach for observing and documenting end-user behaviour with lighting control interfaces does not rely on accepting assertions generated by personal opinions or preconceived notions about these interfaces.

However, there is a trade-off between the amount of information that can be obtained by simultaneously placing end-users in roles of reaction and participation. As the evaluation tool is based on key characteristics of tangible lighting control interfaces, the scope of end-users evaluating them in a more personalised and subjective manner is reduced. Moreover, as end-users have already been made aware of the key characteristics during evaluation, any scope of obtaining additional 
criteria for evaluation or design information about their desirable interfaces is also reduced. A possible mode of countering this trade off would be to differentiate the key characteristics for evaluation at the beginning of the experiment, and then asking participants to suggest any missing additional criteria.

\subsection{Research Structure and Thesis Layout}

The general research structure follows the "informant design framework" developed by Scaife, Rogers, Aldrich, and Davies, which refers to the value of seeking a view from within the end-user domain, while still attempting to go beyond it, to form a general theory (1997, p. 344). The informant design framework was used to design interactive learning environments for children, by placing multiple informants such as children, teachers, software developers, etc., in reaction and participation roles. Therefore interplay between privileged observations from multiple informants is used to obtain feedback for designing the best possible software for teaching children. Each of these informants provided feedback at different phases of the project. Following this example, this thesis research is divided into six phases where informants provide feedback on interface designs either in the form of literature reviews or empirical tests.

In Phase One the emphasis is necessarily on identifying end-user requirements for maximising usability and end-user experience of lighting control interfaces, which in turn might lead to tangible interaction. In Phase Two the emphasis is on the dominant views on tangible interaction to derive principles for designing tangible lighting control interfaces and their key characteristics. In Phase Three, two interactive 
studies are designed where these characteristics are transformed into tools for evaluating the usability and end-user experience of lighting control interfaces and provide feedback for better designs. These tools are then used for the first interactive study where potential end-users are asked to evaluate existing interfaces as well as provide design ideas and suggestions about their most desirable interfaces in Phase Four. Inputs from these sessions are then combined with the principles for designing tangible lighting control interfaces to evolve a prototype, which is subsequently tested by end-users using the second interactive study in Phase Five. Finally, Phase Six reviews the entire thesis research and provides directions for future work.

\subsubsection{Phase One: Define end-user requirements}

The primary goal of Phase One is to identify those end-user requirements that can maximise usability and end-user experience of lighting control interfaces, and may lead to tangible interaction with the lit environment. This phase first identifies how lighting control concepts can be introduced, what levels of comprehension are required by end-users at different points, what the pros and cons of different interfaces might be, and how it might help [directly or indirectly] in tangible interaction with the

lighting. To provide information on lighting controls, Chapter 2 looks at control techniques, strategies and interfaces with respect to the human psychophysiology that may result in tangible interaction and rich sensory experiences with lighting. This phase then analyses end-user requirements and provides inputs for a developmental analysis of the cognitive difficulties likely to be posed by lighting control interfaces. To understand end-user requirements, Chapter 3 reviews literature from the most influential studies on interactive systems. This phase ultimately provides a set of research hypotheses that predict end-user behaviour with lighting control interfaces. Thus even at a preliminary stage, a basis 
is provided to begin sketching out a range of ideas for alternative designs.

\subsubsection{Phase Two: Characterise tangible interfaces}

The primary goal of Phase Two is to derive design principles and key characteristics of tangible lighting control interfaces. This phase begins with a notion that every time an end-user performs a lighting control function with the interface, a conversation is carried out between the enduser and the lit space. This is based on Tidwell's argument that while designing an interface, the designer gets to "script that conversation or at least define its terms" (2005, p. 3). Therefore as lighting control interfaces mediate conversations between end-users and the lit spaces, the aim is to bridge the gap between the user-controlled function and the lighting scenario it represents. This phase provides the point of departure by deriving principles for designing tangible lighting control interfaces, which are then used to evolve their key characteristics. To characterise tangible interfaces, Chapter 4 first studies the dominant views on tangible interaction to derive the principles, and then evolve specific characteristics from these principles. This phase ultimately provides a set of guidelines for designing tangible lighting control interfaces. Thus the underlying lighting control concepts are made easier to understand and the interface is made more accessible to the end-user.

\subsubsection{Phase Three: Design interactive studies}

The primary goal of Phase Three is to design interactive studies that will enable end-users to evaluate interfaces as well as design future interfaces. This phase delves into the scientific survey methods used in behavioural research to devise a strategy for observing end-user behaviour with lighting control interfaces. The strategy is to use 
interactive studies for involving end-users in roles of reaction and participation to evaluate and design interfaces respectively. To design interactive studies, Chapter 5 reviews different scientific methods, procedures and systems used for observing, gathering, analysing and interpreting data on end-user behaviour. This phase ultimately provides a methodology to transform the key characteristics into tools for evaluating interfaces and obtain feedback for future designs. Thus the use of a unique hybrid approach is validated for evaluating and designing interfaces based on end-user feedback.

\subsubsection{Phase Four: Evaluate existing interfaces}

The primary goal of Phase Four is to enable end-users to evaluate existing interfaces for lighting control as well as provide ideas for future designs. This phase activates an existing interface study where potential end-users interact with existing interfaces to perform various lighting control tasks and evaluate them. This study also forms a part of the effort to test the evaluation tools and the research hypotheses as they are being formulated. To evaluate existing interfaces, Chapter 6 describes the setup used for enabling end-users to perform lighting control tasks with existing interfaces, and their evaluated results. This phase ultimately provides end-user feedback on existing interface designs as well as ideas for designing better existing and future tangible interfaces. Thus an analysis is provided on how effective existing interfaces for lighting control and their external representations are, and what the pros and cons of different forms might be.

\subsubsection{Phase Five: Evaluate virtual prototypes}

The primary goal of Phase Five is to design and test a prototype tangible lighting control interface. Preece, Rogers, and Sharp argue that 
prototyping is used to overcome potential client misunderstandings as well as test the technical feasibility of a suggested design (2002, p. 180). The previous phase allows for a better understanding on how to formulate interface characteristics for use with existing interfaces. This phase concerns itself with using the principles for designing tangible lighting control interfaces to design, test and evaluate prototypes with potential end-users. To design and test a tangible interface, Chapter 7 describes the development of virtual prototypes, the setup used for enabling end-users to perform lighting control tasks with these prototypes, and their evaluated results. This phase ultimately provides end-user feedback on tangible interfaces that are primarily focussed on a wide range of interface uses. Thus an analysis is provided on the benefits of better narration of the lighting control functions, feedback, and cues for action.

\subsubsection{Phase Six: Discuss, review and implement}

Chapter 8 reviews the project by drawing overall conclusions from the discussion and analysis, and relates the conclusions back to the overall aim of the work as stated in Section 1.4. Finally, Chapter 9 discusses future trends by providing ideas for future research possibilities, and where excerpts of the project can be handed over to certain select members of the lighting industry for implementation and further validation.

\subsection{Chapter Inference}

By virtue of their interactive nature, lighting control interfaces can help end-users better utilise the flexibility offered by control systems, and thus play an important role in the design of quality lighting environments. The 
complexity posed by existing control interfaces is a problem. To maximise usability and end-user experience of lighting control systems, a new approach to designing control interfaces is sought. This thesis research proposes design principles and key characteristics for maximising the usability and end-user experience of lighting control interfaces, and explores them with a prototype. 


\section{CHAPTER TWO}

\section{Personalising Lighting Controls}

\subsection{Chapter Intent}

Chapter 2 highlights the importance of personalising lighting controls for attaining tangible interaction with the lighting. In Chapter 1, the challenge of designing quality lighting environments with lighting controls is introduced and a new approach for designing tangible lighting control interfaces is sought. Winchip argues that lighting controls include a broad category of techniques and equipment that are designed to enhance an environment and conserve energy (2005, p. 232). However as this thesis research aims to design quality lighting environments by enhancing human sensory experiences, the entire concept of providing lighting controls in terms of its functional meaning [techniques and strategies] and its physical nature [interfaces] needs to be reintroduced. This reintroduction should aim at directing lighting controls towards tangible interaction.

Winchip further argues that planning of controls requires a thorough analysis of the current and future needs of people and their activities in the environment (2005, p. 239). Lam argues that people have a biological need to personalise their private environment and define the territory in which they live and work, which has strong implications for the design of their luminous environment (1977, p. 29). Therefore it is important to understand this biological need in detail and how this may 
lead to tangible interaction. Additionally, the pros and cons of existing lighting control interface designs have to be investigated to categorise and compare their ability at fulfilling people's biological needs. The literature required for performing the overall analysis is derived primarily from the latest editions of books, catalogues, journals and manuals on lighting control technology (Dynalite, 2008; Helvar, 2007; Simpson, 2003). The outlook is towards attaining tangible interaction with the lighting by personalising lighting controls.

Section 2.2 reviews literature to reintroduce and direct existing lighting control techniques, strategies, and interfaces towards tangible interaction. Section 2.3 reviews literature to explain the importance of people's biological need for personalised environments and how this may lead to tangible interaction. Section 2.4 reviews literature to put forward an overview of the psycho-physiological processes involved while fulfilling people's biological need for personalised environments and its implications for designing lighting control interfaces. These literature reviews are then used in Section 2.5 to categorise and analyse the pros and cons of existing lighting control interfaces in fulfilling this biological need. Section 2.6 finally summarises Chapter 2, and draws inferences for understanding end-user requirements in Chapter 3.

\subsection{Lighting Controls: Reintroducing them!}

The techniques, strategies and interfaces used for aesthetic control emerge as the mode for designing quality lighting environments. Designing quality lighting environments is a process of understanding the primary objectives of lighting controls and identifying all the relevant strategies required for achieving tangible interaction. This is based on 
Fields, Wright, and Harrison's argument that designing interaction is a process of understanding the objectives underlying end-user activity, and the strategies that are appropriate to those objectives (1997, p. 165). Chapter 27 of the IESNA Lighting Handbook describes energy management, aesthetics, and code compliance measures as the three major objectives for the use of lighting controls (Rea, 2000, p. 1). Egan and Olgyay further describe that lighting control systems typically sense environmental conditions [time, amount of light] and human intervention [occupancy, motion] to initiate responses that are typically for comfort, energy efficiency and life safety (2002, p. 203). The strategies that meet the objectives of aesthetic controls provide the ability to change spatial functions, and create emotional appeal, offering control of lighting quality, mood, colour, and attitude along with producing significant energy savings (Rea, 2000, p. 1).

However quality lighting environments should enable people to 'configure' their environment by adjusting the lighting. This is based on Hornecker's argument that configurability is essential for providing tangible interaction as it gives control over the environment by enhancing engagement, supporting explorative behaviour or providing thinking aids (2006b, p. 2). This 'configurability' of the environment can be achieved by aesthetic control as it provides the means to adjust the lighting to suit the purpose and/or change the mood while maintaining human visual performance (Rea, 2000, p. 3). For example, adjusting from bright-andgeneral to low-and-accented lighting can configure a working conference room in Bruce's office into an evening dining area. This in turn can enhance engagement and support explorative behaviour of people attending the conference, while providing Bruce with thinking aids to adjust the lighting to suit the evening dinner mood. 


\subsubsection{Aesthetic Control - Techniques}

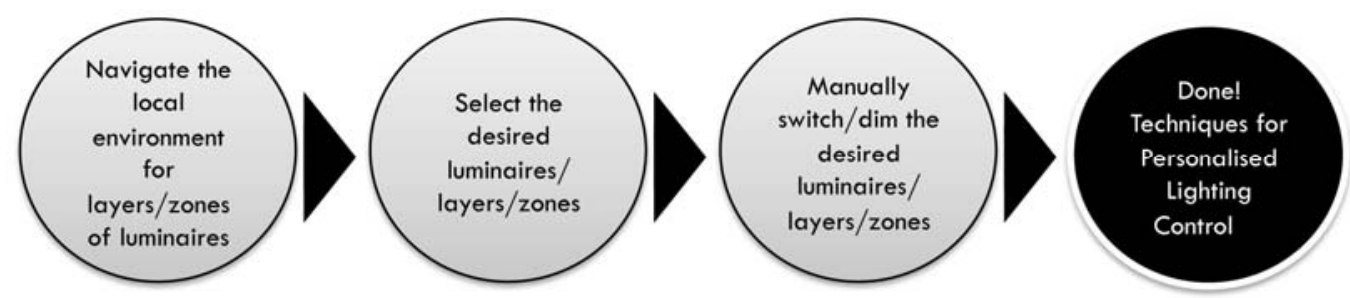

Figure 1 - Aesthetic Control Techniques

Switching and dimming techniques, often being the techniques used for configuring lit environments using smooth transitions in luminous intensities and colours, can provide the techniques required for tangible interaction. The three major lighting control techniques include switching or dimming control, local or central control, and degree of control automation (Rea, 2000, p. 5). Switching control literally switches on and off the electric lighting, while dimming control smoothly and continuously increases or decreases the illuminance in an area or zone to dynamically match visual requirements. A local lighting system is divided into independently controllable areas or zones consisting of different layers of lighting, the size and shape of which is typically dictated by the geometry or the functional needs of the spaces. Central systems generally combine many local zones. Depending upon the spatial requirements, control systems vary greatly in degree of automation, ranging from manually operated to highly automated controls.

Many aesthetic applications require control of illuminance over a wide range by switching and dimming, where dynamic changes can occur rapidly to create excitement or subtly to create a smooth transition between different room functions (Rea, 2000, p. 4). For example, Bruce himself can switch or dim the local lighting layers in the conference room to create the transition into an evening dining area with a manual 
controller. Or his assistant Jenny can adjust the lighting at an appointed time discussed beforehand from a central control unit placed near her desk to bring an element of surprise for the people attending the conference.

\subsubsection{Aesthetic Control - Strategies}

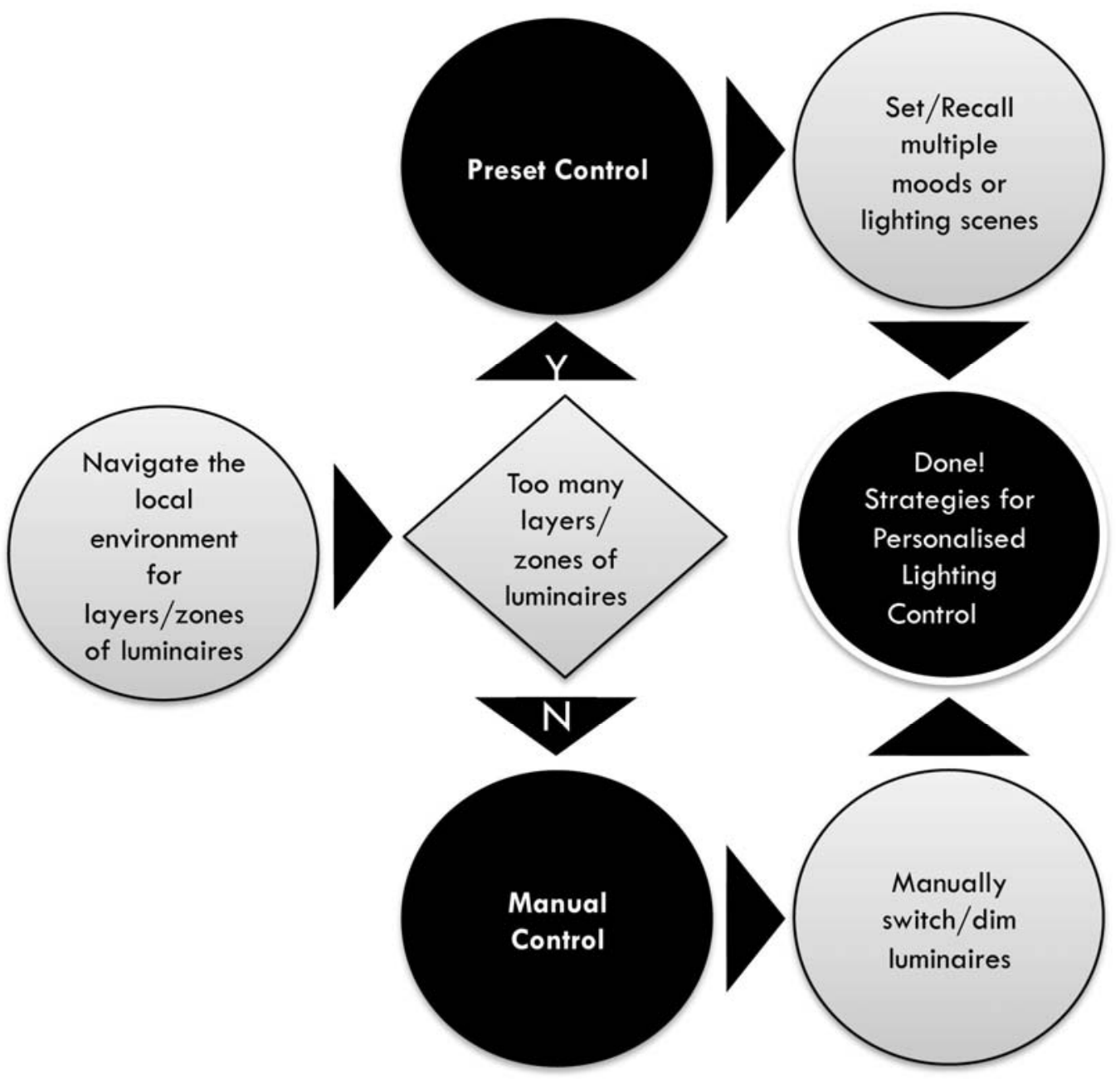

Figure 2 - Aesthetic Control Strategies

Manual and preset controls, often being the strategies used for configuring lit environments, can provide the strategies required for tangible interaction. The strategies used for aesthetic control include 
manual, preset and central control systems (Rea, 2000, p. 4). Manual controls as the name suggests involves the manual operation of switching and dimming functions. A preset scene is established when certain specific luminaires in a space have been grouped to form a layer or zone, and dimmed to provide a specific luminous intensity and/or aesthetic look. This scene can be created or modified by navigating for the available layers or zone of luminaires, selecting the desired layers, grouping and switching or dimming them. Once the desired scenes are set, the process also enables recall of desired scenes. Central dimming systems are the most powerful of the group of dimming options, which can include several forms of manual or preset controls.

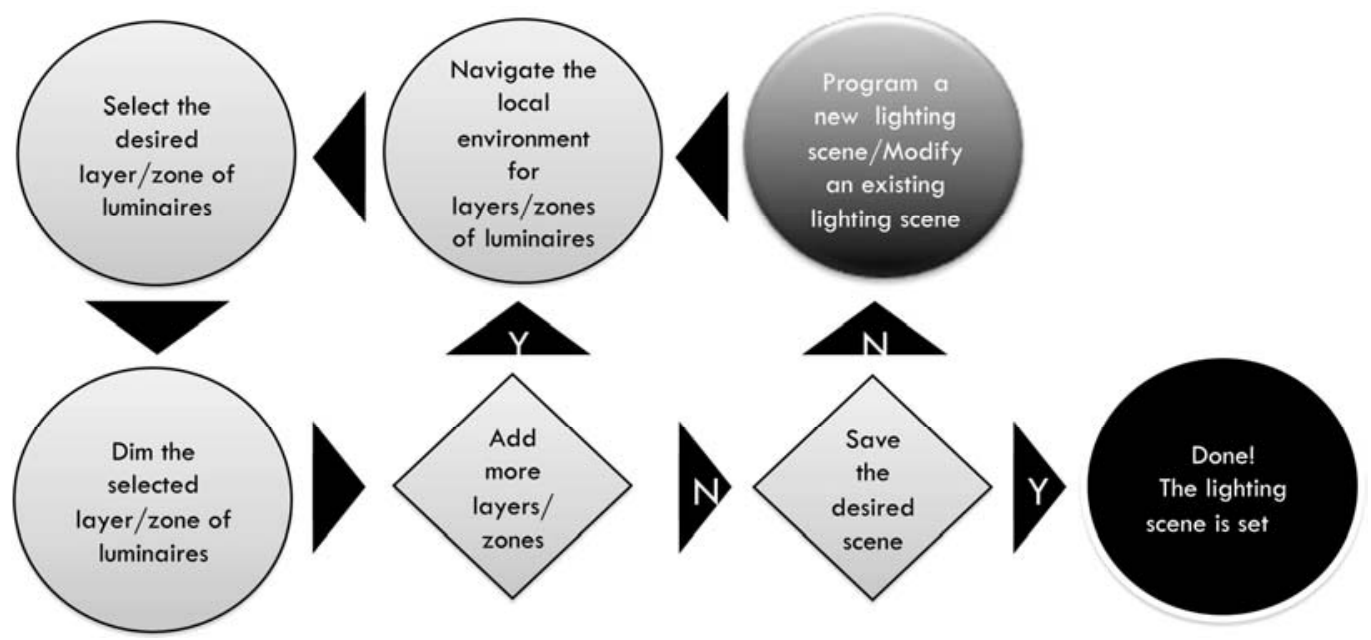

Figure 3 - Preset Control

Although it is a common practice to allow manual control of lighting, some facilities or spaces may require a number of switching or dimming options. Steffy explains that this can lead to wrong selection of light intensity or inappropriate mood, thereby making it annoying and tedious; in such situations, consideration should be given to preset controls (2001, p. 168). Preset controls can actually keep track of switching or dimming controls for all the lights in a space, and allow the specification of preset scenes. For example, instead of manually adjusting every layer of 
lighting to configure the conference room into an evening dining area, Bruce can recall a saved 'Evening Dinner' scene with the desired aesthetic look using a preset controller.

\subsubsection{Aesthetic Control - Interfaces}

Power controllers and sensing devices, often being the visible and usable parts of the switching or dimming system, can provide the interfaces required for tangible interaction. Although the aesthetic control strategies can have different but overlapping sets of hardware requirements, tangible interaction requires interfaces that operate on bodily interaction. This is based on Hornecker's argument that bodily interaction is essential for tangible interaction as it stimulates mental energy, enhances engagement, and is expressive (2006b, p. 1). The three major hardware components of control systems are the Power Controller, the Sensing Device and the Logic Circuit (Rea, 2000, p. 7). The power controller, such as a dimmer, relay, or switch, is the "business end" of a control system that electrically changes the output of the light source. The sensing device, such as an occupancy or motion-sensor, automatically detects the presence of occupants and transmits lighting control information to the logic circuit, which is the intelligence that decides by what means to supply electric lighting, and how much. The power controllers require physical body contact while the sensing devices require bodily movement for switching or dimming, and configuring lit environments. For example, Bruce can touch the keys on the preset controller, or move and wave his hand under a motion-sensor to configure the lighting in the conference room after the meeting. 


\subsection{Personalising Controls: Why is it needed?}

Personalising lit environments with locally available manual and preset controls can improve clarity and ease of understanding the environment by fulfilling people's biological need. Lam argues that human beings share certain needs called "biological information needs: needs to understand the nature and structure of our environment, needs which are rooted in Darwinian drives for survival and security, needs which transcend the scope of aesthetic squabbles based on personal fancy or on merely cultural distinctions between groups of human beings" (1977, p. 5). In order to fulfil this biological information need, people constantly monitor and navigate the environment in which they live and work in for new information on lighting that might be of significance to their activities. Lam further argues that people evaluate an environment according to how well it is structured, organised, and illuminated to satisfy their needs for visual information, which is derived from both the activities in which they choose to engage and the biological information needs related to the very essentials of human nature that are present regardless of the specific activity which holds their attention at any one time (1977, p. 5).

Winchip argues that environments with quality lighting are specifically designed for people, and reflect a skilful application of the principles of design by integrating and layering light into the entire composition (2005, p. 17). Before performing any lighting control task, people have to first navigate the lit environment to evaluate and choose those lighting layers that will be suitable to their activities. Palmer describes our evolutionarily adaptive behaviour to be dependent upon the information we get about the presence and location of objects in our environment, and the opportunities they afford us (1999, p. 6). Krippendorf and Butter discuss a truism that humans surround themselves with objects which they are comfortable with and experience as meaningful (2008, p. 354). 
This conception of meaning recognises that humans create their own environments and distinguish their objects according to what they mean to them, including how they enter the communications about them. Therefore, once the desired layers of lighting are navigated and chosen, people also require information on how to select, group, switch or dim them using the lighting control interface. Egan and Olgyay report that the usability of power controllers as control interfaces can be improved by selecting them according to their control function and locating them where their use will be convenient and predictable - clarity and ease of understanding the switching and dimming functions is crucial (2002, p. 204). For example, although Jenny has a central control unit situated near her desk, Bruce and his partner Ann can better understand the lighting of their respective cabins if local manual controls are provided at each of their desks. Both will have the freedom to choose lighting scenarios suitable to their activities, and Jenny has to only turn off all the lights at the end of a working day.

\subsubsection{Tangible Interaction through Personalisation}

Personalising lit environments with tactile physical interfaces for local manual and preset controls can lead to tangible interaction with the lighting. Egan and Olgyay draw analogies between building control systems and human systems as both sense a condition before initiating an appropriate response (2002, p. 203). Preece, Rogers, and Sharp however argue that in human interaction with systems, vision is considered the most dominant sense for sighted individuals to receive information, followed by hearing and touch (2002, p. 76). Then as sighted individuals, lighting plays a significant role in our behavioural patterns as we receive most of the spatial information to act on through our visual sense. Palmer broadens this viewpoint by stating that besides seeing, all our other senses namely hearing, touching, tasting, and smelling 
participate in this endeavour of providing biological information (1999, p. 6). Additionally, the works of Schmorrow, Stanney, Wilson, and Young on human-system interaction, and Sharma, Pavlovic, and Huang on multimodal human-computer interface, support this viewpoint by identifying that human interaction utilises more than one mode of sensing (2006, p. 1356; 1998, p. 854). Therefore a quality lighting environment should cater to all our other senses along with our visual sense in order to provide rich sensory experiences.

Hornecker, however refers to the physicality of the end-users' body and the physical world for tangible interaction, and argues that our tactile sense is multimodal and is the only active sense, which is not purely receptive: the aesthetics of touching something has immediate emotional responses (2006a, p. 23). Diajadiningrat, Overbeeke, and Wensveen reinforce this by arguing that the "sensory richness of physical objects can act as carriers of meaning in interaction" (2002, p. 285). A study conducted by Maniccia, Rutledge, Rea, and Morrow provides evidence that user-controlled lighting with manual controls leads to high occupant satisfaction (1999, p. 56). Additionally, Chapter 27 of the IESNA Lighting Handbook reports that although automatic controls in the form of occupancy or motion sensors can reduce energy consumption, they are not always effective in terms of occupant response (Rea, 2000, p. 7). Therefore the seemingly simple act of turning on lights at the beginning of a working day regardless of visual need, can have an inference that people want to experience tactile physical interaction with the lighting. But there is no conclusive reason behind end-users' reluctance to turning off lights at the end of the working day, as documented by Moore, Carter, and Slater (2003, p. 54). For example, in the unlikely event of Jenny forgetting to turn off all the lights in Bruce's office at the end of a working day, occupancy-sensors with built-in timer units can be installed 
to turn them off when the office is left unoccupied for a certain period of time.

\subsection{Personalising Controls: A psycho-physiological overview}

The idea behind decomposing the lighting control tasks performed in lit environments into perceptual-cognitive-motor co-ordinations is to understand how people use their cognitive and perceptual-motor skills to satisfy their biological need for personalisation; and what implications this might have in the design of lighting control interfaces. Lam argues that designing lit environments that respond effectively to all the needs of end-users, requires an understanding of the working of the innate, automatic perceptual mechanisms related to the satisfaction of their biological needs (1977, p. 19). Krippendorff and Butter reinforce this by arguing that designing artefacts for human use requires going beyond their forms and decomposing them into sequences of human actions and responses from the artefacts (2008, p. 358). An abstract overview proposed by Card, Moran, and Newell on the information processing sequence that occurs within the human brain, discusses three broad systems (1983, p. 24). Information is first perceived through multiple sensory processors of the perceptual system. This information is then perceptually encoded and processed by the cognitive system to arrive at a decision, which in turn triggers the motor system for a response. For example, Bruce and Ann have recently installed a lighting control system in their plush new residence. All lighting control tasks performed in the residence such as navigation, selection, grouping, switching or dimming will undergo this sequence of perceptual, cognitive and motor processes within their respective psycho-physiological systems. 


\subsubsection{The Perceptual System}

The biological information need can be adequately fulfilled when lighting control information represented on interfaces is perceived via visual and tactile modalities. The physical design and representations on interfaces should maximise perception by providing rich visual information about switching, dimming, programming and recalling desired lighting scenarios. Additionally, interfaces should enable haptic exploration of its operational mechanics. The process of perception can be divided into two stages - the physical reception of the stimuli, and the processing and interpretation of that reception. Within the perceptual system, focus will be on those perceptual sensors that act as input elements while using interfaces for personalised lighting control. Lam argues that the information content of the incoming stimuli, and not their absolute intensity, is a key determinant of perceptual evaluation: quality dominates quantity $(1977$, p. 3). For example, when Bruce wants to program a new lighting scene that is suitable for watching a movie in the living room, rich visual information about the available lighting layers has to be provided by the interface for navigation, selection and grouping along with functions of switching and dimming.

Schmorrow, Stanney, Wilson, and Young argue that a considerable amount of information can be perceived if it is allocated across multiple sensory systems (2006, p. 1365). Tegin and Wikander argue that visual information needs to be complemented with haptic information to maximise perception (2005, p. 65). Keyson reinforces this by reporting that the tactile feedback of early analogue technologies through physical mechanisms such as graspable knobs and dials, has been largely replaced by digital electronic buttons and visual displays, which in turn has led to higher demands on the visual system (1997, p. 4/1). A study carried out by Escuyer and Fontoynont further reports that end-users 
found rotary dimmers "less cumbersome" than remote controls (2001, p. 93). Gehnen argues that although it is important that the operating buttons on a device are logically arranged and the software menu is clear, a product only becomes successful when it has a further tactile quality: it has to feel good to touch, to operate and to hold in your hand (2008, p. 19). Kinoshita, Aida, and Mori have classified the sensing pattern of the tactile system under grasping by shifting the gripping position, and groping to determine the surface shape of the object coming into contact (1975, p. 243). For example, Ann can grasp and grope the control handle of the dimmer in the bedroom with her hand to quantitatively detect its surface shape, size and form, or determine its manipulability by shifting the gripping position.

\subsubsection{The Cognitive System}

The biological information need can be adequately fulfilled when lighting control information on interfaces is appropriately presented, sequenced, paced and delegated. The physical design and information represented on interfaces should be easily recognisable, and avoid complex cognitive processing while performing the switching, dimming and preset control tasks. Schmorrow, Stanney, Wilson, and Young argue that an excessive amount of cognitively demanding tasks imposed on the end-user can lead to annoyance due to human information overload (2006, p. 1369). They further argue that instead of overwhelming people with demanding control tasks, interfaces for performing these tasks should seek to achieve "cognitive congeniality" by the appropriate "presentation, sequencing, pacing, and delegation" of tasks (p. 1369). Additionally, Thomassen argues that the interface design should involve a "three-level navigation process" for "storage, deletion and retrieval" of control information in human memory that is set as a standard (2003, p. 65). Atkinson and Shiffrin have proposed a psychological model for the 
structure of human memory in a sequence of three stages: sensory memory, short-term or working memory, and long-term memory (1968). For example, the visual and tactile information provided by the interface first enters Bruce and Ann's memory where it is either stored for future retrieval, or deleted if found redundant and unnecessary.

\subsubsection{The Cognitive System - Sensory and Short-term memories}

Presentation of task-relevant information on lighting control should be most favourable for sensory perception and processing. Sensory memory is an area in the brain where sensory stimuli are stored as a buffer for a short time before they are processed and passed on to the short-term memory. Atkinson and Shiffrin argue that sensory memory allows the sensation of a sight or a touch to linger momentarily after the actual sensory stimulation has ceased, and is responsible for encoding the visual or haptic information received and converting it to a usable mental form (1968). For example, there is a different sensory memory system for each of Bruce and Ann's senses - iconic memory for visual stimuli, and haptic memory for tactile stimuli. The stimuli are triggered either by images or touch received by the iconic or haptic memory, stay there for a limited amount of time, and are passed on to the short-term memory.

Short-term or working memory is considered a temporary active storage area, where information that has been perceived by sensory memory is manipulated and maintained, for executing simple and complex tasks. Baddeley and Logie argue that short-term memory acts as a scratch-pad for the temporary recall of information under process (1999). For example, this allows Bruce to comprehend and mentally represent the lighting scenario required for watching a movie, to retain immediate information about the control keys he used to program this scene and to support the acquisition of new knowledge about these control keys. Now 
given the limited capacity of Bruce and Ann's sensory and short-term memories, the lighting control information on interfaces should be strategically allocated to the visual and tactile sensory systems for increased input capacity and maximising sensory perception. The load on Bruce's sensory memory can only be relieved when consideration is given to the suitability of each of the visual and tactile sensory modalities for representing information on programming a lighting scene. Images or icons can be used to represent the different lighting layers available in the living room to enable navigation with the visual sense, and a tactile procedure can be used for selection, grouping and dimming. This in turn will support the simultaneous processing of competing control tasks such as navigating and dimming, while maintaining control information demands within Bruce's short-term memory capacity.

\subsubsection{The Cognitive System - Attention}

Sequencing of lighting control tasks should be systematic with subtle cues for prioritising lighting control information and moderating attention. Attention is the cognitive process of selectively concentrating on one aspect of the environment at a point in time, while ignoring the range of other possibilities available. Sohlberg and Mateer's model for evaluating attention describes five different types of activities (1989). Focussed attention is the ability to respond discretely to specific visual or tactile stimuli. Sustained attention is the ability to maintain consistent behavioural response during continuous and repetitive lighting control activity. Selective attention is the capacity to maintain a behavioural or cognitive set in the face of distracting or competing stimuli. Alternating attention is the capacity for mental flexibility that allows people to shift their focus of attention and move between different lighting control tasks having different cognitive requirements. Distributed attention being the highest level of attention is the ability to respond simultaneously to 
multiple lighting control tasks. Human performance on a wide range of tasks is enhanced by attention as it minimises distractions, and facilitates access to awareness by filtering out irrelevant stimuli. For example, the task of programming the 'Movie scene' should be in the sequential order of navigation, selection, grouping and switching or dimming. This can assist in prioritising incoming information by sequencing cues about the different lighting layers and the luminous intensity level required for each layer according to priority, thereby directing and moderating Bruce's attention.

Executive attention comes into play in situations where cognitive decisions independent of incoming sensory stimuli have to be made for coherent behaviour in order to rein attention. Norman and Shallice argue that executive attention is heavily involved in handling novel situations involving contextual ambiguity of planning or decision making, error correction, or trouble shooting (2000). For example, the effort on Bruce's executive attention can be reduced when the recall of contextual information for achieving the movie scene is cued with incoming information for optimal interpretation. The interface can provide subtle clues about selecting the lighting layers and their luminous intensity levels for creating the movie scene, which in turn will enable easier error correction in case a wrong selection is made.

\subsubsection{The Cognitive System - Long-term memory}

The three-level navigation procedure of storage, deletion and retrieval should decrease pressure on the long-term memory process by enabling recognition rather than recall of lighting control information. Long-term memory is intended for storage of information over a long time and can be perceived as the main resource for high-capacity storage of control information. Information from the working memory is transferred to long- 
term memory through the process of rehearsal and meaningful association, and unlike in working memory there is little decay. Dubuc typically divides long-term memory into two major headings: Procedural memory and Declarative memory (2002). Procedural memory refers to the use of interface objects or movements of the body, such as how exactly to use a manual-dimming interface. Declarative memory on the other hand is further subdivided into two types namely, Episodic and Semantic. Episodic memory represents memory of events and experiences in a serial form, and enables people to recall and reconstruct passed lighting scenarios at any given point of time. Semantic memory is a structured record of facts, concepts and skills that have been acquired, and enable people to make inferences about a lighting control function, generalise and get into detail.

Thomassen argues that the three main activities that take place in the long-term memory process are information storage, deletion, and retrieval (2003, p. 63). Storage of information in long-term memory is caused by the repeated exposure to a visual or tactile stimulus through the rehearsal of a lighting control task. Deletion of information can be caused either due to decay or interference. Decay happens when stored information about performing a particular lighting control task expires due to lack of constant use. Interference happens when new information about performing a particular lighting control task causes loss of old information. Nonetheless, it is unsettled whether information is actually ever forgotten or whether it becomes increasingly difficult to access certain items from memory. Retrieval of information can be done either by recall or recognition. Long-term memory is affected by the emotional, representational and structural quality of information. When information about performing a particular control task is well organised and structured, it can be easily recalled. For that matter, when the 
represented information about performing a particular lighting control task provides knowledge that it has been seen before, it leads to easier recognition. For example, information on existing interfaces is normally displayed through typography and iconography. Typography refers to the various features of the alphanumeric characters conveying information about lighting control both individually and collectively. Iconography is used for easier recognition, and the ability to learn the use of a symbol or an icon. As storage for remembrance occurs only for meaningful information, it is easier for Bruce to remember words or icons related to functions like 'Movie Scene' or 'Evening Dinner Scene' than words related to concepts like 'Low-level', which are more abstract and less meaningful. As emotional factors affect Bruce's long-term memory, positive information about a particular 'happy' mood or scene is less likely to be forgotten, as this is of an emotional nature.

\subsubsection{The Motor System}

The biological information need can be adequately fulfilled when lighting control interfaces require simple body movements for operation. The physical designs and information represented on interfaces should elicit faster reaction times by providing visual clues in a sequential order about its function and operation. The last part of the input-processingoutput cycle, called the motor system covers the output area of human behavioural response mainly involving bodily movement. However, from the entire body of knowledge on the motor system, only those areas that are relevant to this thesis research are discussed. Thomassen argues that a focus on the reaction time required for human responses gives an insight into designing interfaces (2003, pp. 71-72). Psychologists have named three basic types of reaction times namely simple, recognition, and complex reaction times (Luce, 1986; Welford, 1980). These three 
basic types of reaction times determine the type of actions performed by humans.

Simple reaction time is defined as the time required to detect the presence of one particular stimulus and responding to it by performing a simple task that requires the same response stimulus. For example, the time required by Ann to search for the light switch in the attic and immediately switch it on upon entering. Although in practice she will have to respond to several of those simple reactions, this does not influence the reaction time to a degree significant enough to disturb. Recognition reaction time is defined as the time required for detecting the presence of several stimuli, and responding to only one correct stimulus and ignoring the rest. For example, the time required by Jenny to recognise and press the 'Off' button to turn off all the lights from the central control panel with several buttons, while leaving the office at the end of each working day. Choice reaction time is defined as the time required to detect the presence of several incoming stimuli and address all of them, but all requiring a different response which obviously slows down the reaction time. For example, the time required by the butler Alfred, to recognise and press several buttons to manually switch-on the lighting and ventilation, upon entering Bruce's office on a weekend for cleaning. However he can learn to adapt to some of those recurring reactions with rehearsal.

The pioneering study of Donders shows that simple reaction time is shorter than recognition reaction time, and that choice reaction time is the longest of all (1969). Miller and Low have determined that the time required for a motor response is the same in all three types of reaction times, implying that the differences in reaction time are due to processing time (2001). However, Thomassen argues that a simple body movement 
will obviously have less impact on the increase of the reaction time compared to complex types of body movements (2003, p. 72). Additionally, Kosinski assesses that reaction time to touch is faster than sight, and visual stimuli that are longer in duration elicit faster reaction times. Brebner and Welford argue that faster reaction times can be achieved by warning about the arrival of a stimulus (1980). For example, as Ann's simple tactile reaction time is faster compared to visual reaction time, the simple action of dimming requires more tactile information with a control handle that has higher tactile properties such as larger surface area or texture. Welford argues that in case of several incoming stimuli, faster reaction times can be achieved by a sequential run of several identical stimuli rather than having them in a mixed order (1980). For example, as an increase of reaction time happens when Bruce is not prepared for new or infrequent signals, each new control function of selection, grouping and dimming requires a different cue. As Jenny is compatible with repetitive action of turning off the office lights from the central control unit at the end of each day, her mind is prepared for some reactions. However, her preparedness can be increased with a short warning before the proposed action such a beeping light on the 'Off' switch at a specified office closing time each day. The more Alfred is practiced with the central control unit, the lesser will be his choice reaction time, and other alternative responses will have less influence on his reaction time.

\subsection{Personalising Controls: Investigating interfaces}

The operation of existing lighting control interfaces requires a combination of people's cognitive and perceptual-motor skills to fulfil their biological need. Steffy classifies existing manually-operated 
lighting control interfaces under Pushbutton, Rocker, Slider or Toggle for conventional on/off switching, and Pushbutton, Rotary or Slider for dimming (2001, p. 166). They can range between simple electromechanical interfaces that are used in our day-to-day switching and dimming activities, to the latest trend of sophisticated digital interfaces. Digital interfaces provide the basic functionality of switches, but instead of mechanically opening and closing the circuit to switch the lighting, they send a digital control signal over low-voltage wiring to the control panel or power switching device, which switches the load (DiLouie). Control functions performed with digital control systems require separate control channels for performing the function. Manual controls in order to be effective should be simple and convenient to use, with a minimum number of control channels to avoid confusing choices; preset control systems should allow for several lighting channels to be programmed and controlled simultaneously in order to provide multiple moods or scenes (Rea, 2000, pp. 4-5). The digital interfaces enable the incorporation of greater functionality, such as multiple buttons for on/off switching, preset scene control and dimming. The panel range for digital interfaces involving haptic operational mechanics consists of Pushbutton, Rotary, Slider and Touch options. Additionally, digital control panels can be fitted with infrared receivers [IR] for use with compatible hand-held pushbutton remote controls.

Sections 2.2 and 2.3 have determined that tactile physical interfaces for locally available manual and preset controls can be used for personalising lit environments thereby attaining tangible interaction with the lighting. However, Section 2.4 clearly shows that the physical designs and information represented on lighting control interfaces as well as their operational mechanics play a major role in fulfilling the biological information need. Jensen, Buur, and Diajadiningrat argue that currently, 
the actions required by interfaces [for personalised lighting control] tend to be limited to pushing, rotating, and sliding (2005, p. 9). Buur, Jensen, and Diajadiningrat further argue that locating the keys "sufficiently fast" is the main challenge with buttons and is solely a cognitive effort, whereas the pushing itself is a "monotonous string of motorically trivial actions" (2004, p. 186). Winchip states that system software features of touch interfaces allow customisation of graphics that depict the lighting system on floor plans, elevations, or any other illustration that assists in visualisation and interpretation of spatial illumination which again requires cognitive skills (2005, p. 236). Therefore existing lighting control interfaces require a high degree of cognitive skills, and to a lesser degree of perceptual-motor skills. The categorised comparison of various existing interfaces in terms of catering to people's cognitive and perceptual-motor skills is listed in Tables 1 and 2.

\subsection{Chapter Inference}

Chapter 2 has identified a number of influential factors in terms of techniques, strategies, and interfaces for attaining tangible interaction with the lighting. A combination of locally available manual and preset controls for switching and dimming, requiring bodily interaction with power controllers or sensing devices is presented as an ideal strategy for configuring lit environments. Literature reveals that people have biological information needs for learning about their environments, and are more conducive and responsive to personalised environments. Therefore people can personalise their immediate lit environment by changing the luminous intensity or colour with local manual controls, as well as setting and recalling multiple moods or scenes with local preset controls. The key feature of designing interfaces for personalised 
lighting controls is to determine which control information would be conveyed via which modality. Once the modalities conveying the desired control information are determined, one has to find ways for maximising the perception and processing of this information. Information about personalised lighting control is perceived through visual and tactile modalities, which is then processed for people to react on. Therefore adequate visual and tactile information about control functions has to be provided by the interface for fulfilling this biological information need. This enhances the simultaneous processing of competing control tasks by strategically allocating lighting control information across the visual and tactile modalities thereby maintaining lighting control information demands within the sensory and short-term memory capacities.

The order of sequence for achieving a desired lighting scenario should enable easier recognition and remembrance thereby maximising the three-level navigation procedure of storage, deletion and retrieval. More importantly, the presentation of this information, along with the sequencing, pacing and delegation of these control tasks should lead to cognitive congeniality, as discussed in Section 2.4. Of significance, a whole range of digitally operated interfaces is available for localised manual and preset controls, namely Pushbutton, Rotary, Slider or Touch. People can configure lit environments by the haptic direct manipulation of any of these interfaces. All these interfaces are compatible with IR Remote Controls, which can provide end-users with the flexibility of placing them anywhere and controlling the lighting from any location depending upon people's requirements or convenience. The informationbased actions performed with these interfaces are primarily determined by people's cognitive and perceptual-motor skills. However, a preliminary analysis based on the literature review shows that each of 
these interfaces has its pros and cons in terms of adequately fulfilling the biological information need as categorised in Tables 1 and 2 .

In Chapter 2, the focus is on personalising lighting controls and directing lighting control tasks towards tangible interaction. However, the design development of tangible lighting control interfaces requires further investigation to identify the exact requirements for end-user interface with personalised lighting controls. In particular, investigations are required to understand the fundamentals of end-user interaction. Thus, Chapter 3 will further review literature to investigate the fundamentals of end-user interaction and hypothesise end-user requirements, while Chapter 4 will use this information to develop design principles and key characteristics of tangible lighting control interfaces. 


\begin{tabular}{|c|c|c|c|}
\hline Interface & Illustration & Cognitive skills & $\begin{array}{c}\text { Perceptual-Motor } \\
\text { skills }\end{array}$ \\
\hline $\begin{array}{l}\text { Type: Pushbutton } \\
\text { dimmer } \\
\text { Manufacturer: Lutron } \\
\text { Model: Rania } \\
\text { Source: } \\
\text { http://www.lutron.com }\end{array}$ & $I$ & $\begin{array}{l}\text { Pros: } \\
\text { - Easy to recognise } \\
\text { increase/decrease } \\
\text { functions } \\
\text { - Easy to accurately } \\
\text { select desired luminous } \\
\text { intensity levels }\end{array}$ & $\begin{array}{l}\text { Pros: } \\
\text { - Affords pushing } \\
\text { - Luminous display } \\
\text { provides instantaneous } \\
\text { response } \\
\text { Cons: } \\
\text { - No graspable } \\
\text { qualities } \\
\text { - Inadequate material } \\
\text { qualities e.g. surface } \\
\text { texture }\end{array}$ \\
\hline $\begin{array}{l}\text { Type: Rotary dimmer } \\
\text { Manufacturer: Lutron } \\
\text { Model: Rotary } \\
\text { Source: } \\
\text { http://www.lutron.com }\end{array}$ & & $\begin{array}{l}\text { Cons: } \\
\text { - Difficult to accurately } \\
\text { select desired luminous } \\
\text { intensity levels }\end{array}$ & $\begin{array}{l}\text { Pros: } \\
\text { - Affords grasping and } \\
\text { rotating } \\
\text { - Easy to grasp control } \\
\text { handle } \\
\text { Cons: } \\
\text { - Inadequate material } \\
\text { qualities e.g. surface } \\
\text { texture }\end{array}$ \\
\hline $\begin{array}{l}\text { Type: Slide dimmer } \\
\text { Manufacturer: Lutron } \\
\text { Model: Lyneo } \\
\text { Source: } \\
\text { http://www.lutron.com }\end{array}$ & $\begin{array}{l}1 \\
\text { I } \\
1\end{array}$ & $\begin{array}{l}\text { Pros: } \\
\text { - Easy to accurately } \\
\text { select desired luminous } \\
\text { intensity levels }\end{array}$ & $\begin{array}{l}\text { Pros: } \\
\text { - Affords grasping and } \\
\text { sliding } \\
\text { Cons: } \\
\text { - Difficult to grasp } \\
\text { control handle } \\
\text { - Inadequate material } \\
\text { qualities e.g. surface } \\
\text { texture }\end{array}$ \\
\hline $\begin{array}{l}\text { Type: Pushbutton } \\
\text { presets } \\
\text { Manufacturer: Dynalite } \\
\text { Model: DLP550 } \\
\text { Source: } \\
\text { http://www.dynalite- } \\
\text { online.com }\end{array}$ & $\div$ & $\begin{array}{l}\text { Cons: } \\
\text { - Difficult to accurately } \\
\text { select desired preset } \\
\text { scenes }\end{array}$ & $\begin{array}{l}\text { Pros: } \\
\text { - Affords pushing }\end{array}$ \\
\hline
\end{tabular}

Table 1 - Pros and cons of existing interfaces 


\begin{tabular}{|c|c|c|c|}
\hline Interface & Illustration & Cognitive skills & $\begin{array}{c}\text { Perceptual-Motor } \\
\text { skills }\end{array}$ \\
\hline $\begin{array}{l}\text { Type: Pushbutton } \\
\text { presets } \\
\text { Manufacturer: Dynalite } \\
\text { Model: DRP } \\
\text { Source: } \\
\text { http://www.dynalite- } \\
\text { online.com }\end{array}$ & & $\begin{array}{l}\text { Pros: } \\
\text { - Easy to recognise } \\
\text { increase/decrease } \\
\text { functions } \\
\text { - Easy to accurately } \\
\text { select desired preset } \\
\text { scenes } \\
\text { Cons: } \\
\text { - Difficult to accurately } \\
\text { select desired luminous } \\
\text { intensity levels } \\
\text { - Difficult to relate } \\
\text { preset scenes to text } \\
\text { descriptions }\end{array}$ & $\begin{array}{l}\text { Pros: } \\
\text { - Affords pushing }\end{array}$ \\
\hline $\begin{array}{l}\text { Type: IR Remote } \\
\text { Control } \\
\text { Manufacturer: Helvar } \\
\text { Model: digidim } 303 \\
\text { Source: } \\
\text { http://www.helvar.com }\end{array}$ & & $\begin{array}{l}\text { Pros: } \\
\text { - Easy to recognise } \\
\text { on/off and } \\
\text { increase/decrease } \\
\text { functions } \\
\text { Cons: } \\
\text { - Difficult to accurately } \\
\text { select desired preset } \\
\text { scenes and luminous } \\
\text { intensity levels } \\
\text { - Difficult to relate } \\
\text { preset scenes to } \\
\text { numeric descriptions }\end{array}$ & $\begin{array}{l}\text { Pros: } \\
\text { - Affords pushing } \\
\text { - Entire interface is } \\
\text { graspable }\end{array}$ \\
\hline $\begin{array}{l}\text { Type: Touch presets } \\
\text { Manufacturer: Dynalite } \\
\text { Model: DTP } 160 \\
\text { Source: } \\
\text { http://www.dynalite- } \\
\text { online.com }\end{array}$ & $\frac{\mathrm{z}}{\mathrm{z}} \mathrm{z} \mathrm{z}$ & $\begin{array}{l}\text { Pros: } \\
\text { - Easy to accurately } \\
\text { select desired luminous } \\
\text { intensity levels } \\
\text { Cons: } \\
\text { - Difficult to understand } \\
\text { which slider is meant to } \\
\text { control which layer of } \\
\text { lighting }\end{array}$ & $\begin{array}{l}\text { Pros: } \\
\text { - Affords touch-tap } \\
\text { and touch-slide }\end{array}$ \\
\hline
\end{tabular}

Table 2 - Pros and cons of existing interfaces [contd.] 


\section{CHAPTER THREE}

\section{Understanding End-user Requirements}

\subsection{Chapter Intent}

Chapter 3 develops understanding of and hypotheses for end-user requirements for interface with personalised lighting controls. The literature reviews done in Chapter 2 have revealed the importance of personalising lit environments, and how cognitive and perceptual-motor skills are used in fulfilling human biological needs for learning about their environment. Overbeeke, Diajadiningrat, Hummels, and Wensveen however, argue that products should respect human skills on three levels, "the wholly [sic] trinity of interaction: cognitive skills, perceptual-motor skills and emotional skills; in other words, knowing, doing and feeling" (2002, p. 9). Diajadiningrat, Wensveen, Frens, and Overbeeke further argue that perceptual-motor skills and emotional skills are linked as enrichment of actions and challenging end-users' motor skills can lead to rich sensory experiences (2004, p. 297). This implies that cognitive and perceptual-motor skills are used for meeting usability requirements of quickly and effectively learning to use the interface, while perceptualmotor and emotional skills are used for meeting end-user experience requirements of experiencing sense-rich interactive properties of the interface. As this thesis research aims to design quality lighting environments, both usability and end-user experience goals of interaction have to be met. Preece, Rogers, and Sharp however, argue that a trade-off between usability and end-user experience goals is important 
as it creates awareness of the consequences of pursuing different combinations of them in relation to fulfilling different end-user requirements (2002, p. 20).

Krippendorff and Butter have differentiated three qualities of experiences for human interfaces with technology: "Recognition, Exploration, and Reliance" (2008, pp. 360-362). Recognition, more accurately, "re-cognition or cognising something again" refers to the stage in which end-users categorise interfaces according to what they could afford them to do, and thus requires cognitive and perceptualmotor skills (p. 360). Exploration "follows recognition," and describes the stage during which end-users search for ways to handle the interface, and thus requires perceptual-motor and emotional skills; while recognition "presupposes familiar forms," exploration leads to "expectations about the sequence of interactions" (p. 361). Reliance is the stage in which endusers have mastered the interface and "proceed naturally, seamlessly and flawlessly;" however, "reliance can be disrupted" bringing end-users back to the need for explorations of alternative ways, and thus requires all three skills (p. 361). This implies that usability and end-user experience requirements should be considered at recognition and exploration stages respectively, while a combination of the two should be considered at the reliance stage. The requirements for recognition, exploration, and reliance are derived from a mix of theory-based knowledge from literature, practical experience and common sense. The outlook is towards a paradigm shift - from primarily passive to interactive lighting controls that can substantially enhance end-user interaction.

End-user requirements for interface with personalised lighting controls are hypothesised in relation to recognition, exploration, and reliance in 
Sections 3.2, 3.3, and 3.4 respectively. These requirements have a propensity to be written as prescriptive statements assuming what endusers require when performing lighting control tasks. These requirements are not intended to specify how to design an interface, but act more like reminders while designing. Section 3.5 then reviews literature to show how the three stages of interaction and their requirements relate to the different attributes of end-users. Section 3.6 finally summarises Chapter 3 , and draws inferences for characterising tangible interfaces in Chapter 4.

\subsection{End-user Recognition}

Hypothesis \# 1 : End-users learn to use a lighting control interface quickly and effectively when its physical design richly represents its intended control function and operational mechanics. Krippendorff and Butter argue that recognising what the artefact is, will lead end-users into approaching, ignoring, or avoiding it, as without a clue to how the interface can help them, it is not likely to come into use (2008, p. 360).

Hypothesis \# 2: End-users learn to use a lighting control interface quickly and effectively when the iconography and typography depicted on the interface richly represents luminous colours or intensities, and lighting layers or scenes. Preece, Rogers, and Sharp argue that recognition should be encouraged while designing interfaces rather than recall, by using icons and consistently placed objects (2002, p. 83).

\subsubsection{Recognition Requirements - Hypothesis \# 1}

End-users require visibly clear signals on what the lighting control interface can do to help them achieve their desired lighting scenarios. Simon describes satisfice [a portmanteau of "satisfy" and "suffice"] as a 
decision-making rationalised behaviour of people to meet criteria for adequacy, rather than to identify an optimal solution (1957). Tidwell argues that this naturalistic rational behaviour of accepting "good enough" instead of "best" is responsible for end-users' tendency of picking and trying the markedly visible control options presented by the interface first, even if it is wrong (2005, p. 12). Poor visibility and lack of conceptual modelling can lead to difficulty in understanding the operational use and function of the interface (Norman, 1990 c1988, p. 19). For example, when Ann wants to dim the lights in the bedroom before taking an afternoon nap, the operation of the interface can become mysterious and difficult when control functions are invisible and hidden from sight. Conceptual modelling allows prediction of the effects of actions with the interface (Norman, 1990 c1988, p. 13). For example, without a good model, Ann will operate the interface by rote without fully appreciating the results, as she is not aware of what effects can be expected.

End-users require perceptually obvious and strong visual clues from the physical design of the lighting control interface about its operational use. Affordance or perceived affordance refers to the perceived and actual properties of the interface, primarily those fundamental properties that determine how it could possibly be used (Norman, 1990 cl988, p. 9). For example, the physical design of the interface can provide Ann with strong visual clues about where to grasp and how to operate it without any requirements of pictures, labels, or instructions.

End-users require controls and displays exploiting natural mapping to show the set of possible actions to understand the operational mechanics of the lighting control interface. Mapping is a technical term meaning the relationship between the controls and their movements, and the results in 
the world (Norman, 1990 cl988, p. 23). Bordass, Leaman, and Bunn argue that mechanical controls that are direct acting, often map naturally onto the physical layout of the devices they control (2007a, p. 8). For example, by taking the right advantage of mapping, Ann will know what to do with interface just by looking at it.

\subsubsection{Recognition Requirements - Hypothesis \# 2}

End-users require information that is within their understanding at a normal ability to use the lighting control interface. Control panels for manually operated interfaces should be clearly and permanently labelled (Rea, 2000, p. 4). For example, if Bruce's interface relies on typography, it should be clear and provide accurate information about the available lighting control functions. The controls for different operations should be clearly labelled with texts describing what can be done, and what can be achieved for setting a particular lighting scenario in his living room. Preece, Rogers, and Sharp argue that consistency refers to designing interfaces that have similar operations, and use similar elements for achieving similar tasks (2002, p. 24). For example, a benefit of having a consistent interface is that Bruce will have to learn only a single mode of operation that is applicable to all aspects of performing that function such as selection or dimming of a desired lighting layer in the living room.

End-users require visible metaphors to compare the functionality of the lighting control interface to an existing interface, which they are familiar with. Krippendorff and Butter discuss a semantic approach in product design which starts with semantics and cognition, where the product uses the knowledge and experience of the end-user to communicate information through symbols and signs (1984, p. 6). Diajadiningrat, Overbeeke, and Wensveen argue that this approach leads to the use of 
iconography and representation where appearance of the product and its controls become signs, often using control panels labelled with icons or may be icons themselves (2002, p. 288). Escuyer and Fontoynont report that the use of remote controls for lighting systems involving two or three light sources can be simplified by using keys with symbols "+" and "-" for each light source, or a layout with fewer keys, combined with inscriptions (2001, p. 93). For example, the use of graphical representations for the lighting layers along with luminous intensity and colour control will prevent Bruce from selecting incorrect options, and thereby reduce chances of making mistakes while programming a scene for watching movies in the living room. The iconography on the interface can match the learned and supposed meaning of the control function itself, apart from being recognised by him as a specific symbol/icon for performing that control function. Preece, Rogers, and Sharp however, argue that the use of different kinds of graphical representations alone can constrain end-users' interpretation of problem or information space (2002, p. 22). For example, Bruce can quickly and effectively interpret the saved preset 'Movie Scene' when a combination of typographic texts and graphical icons meaningfully and naturally describe the scene.

End-users require a mental model that naturally maps with the lighting layers of the lit environment to mentally simulate the operation of the lighting control interface. Conceptual models can provide end-users with a clear and unambiguous illustration of the system setup, about what each control is responsible for (Norman, 1990 c1988, p. 16). Natural mapping takes advantage of spatial analogies and arrangements of controls, which leads to immediate understanding of their actions in the real world (Norman, 1990 c1988, p. 75). Diajadiningrat, Overbeeke, and Wensveen argue that architectural planning lends itself to natural mapping, as controllable elements like lighting have spatial meaning in 
the physical world (2002, p. 289). Preece, Rogers, and Sharp further argue that realistic representations of spatial elements readily tap into end-users' understanding of the physical world (2002, p. 66). For example, Bruce will find it difficult to program or recall the moviewatching scene when the lighting control system image about the living room is inappropriate or incoherent. Representations of lighting layers mapped in relationship to their resultant lighting control effects in the living room can enable him to easily recognise the underlying lighting control concepts. Making decisions about selection and grouping of these layers, or switching and dimming them to achieve a desired luminous colour or intensity will become easier with images or icons that naturally map these functions.

\subsection{End-user Exploration}

Hypothesis \# 3: End-users experience the graspable and material properties of an interface when its control handles provide rich haptic responses that appeal to the sense of touch. Preece, Rogers, and Sharp argue that exploration is encouraged when designs of interfaces constrain and guide end-users to select appropriate actions (2002, p. 87).

Hypothesis \# 4: End-users prefer an interface with control handles that offer easy manipulation by an effortless shift in the gripping position. Krippendorff and Butter argue that exploration involves attending to how the interface is handled in order to bring about the desirable results (2008, p. 361$)$. 
Hypothesis \# 5: End-users experience the conversational property of an interface when it provides rich visual responses by a synchronic visual link to the changes in spatial illumination. Preece, Rogers, and Sharp argue that exploration is encouraged when representations and abstractions are dynamically linked in the designs of interfaces (2002, p. 87).

\subsubsection{Exploration Requirements - Hypothesis \# 3}

End-users require a 'feel' of the physical set-up of the lighting control interface to decide the modality used for experiencing its interactive quality. The concept of constraints refers to determining ways of restricting the kind of interaction that can take place at a given moment (Norman, 1990 cl988, p. 60). For example, Ann's interaction with the interface while dimming the lights in the bedroom can be restricted and guided with the shape and size of its control handle along with its material properties. The interface can embody a mechanism that enables physical exploration as well as informing her about the results of the process of dimming. The shape of the control handle and its material properties will restrict and guide her actions respectively, such as a rotary dial with textural properties on its spherical surface will only afford grasping and rotation.

\subsubsection{Exploration Requirements - Hypothesis \# 4}

End-users require a 'feel' of the manipulation properties of the lighting control interface to experience its operational mechanics. Tegin and Wikander state that forces and torques can be sensed to experience what type of contact it is and derive tactile information about the object's inherent properties, such as stiffness or compliance (2005, p. 65). For example, the stiffness or compliance of the interface's control handle will enable Ann to experience its interactive and manipulative property. 
Dimming lights in the bedroom will be a pleasure if it requires a singular effortless action.

\subsubsection{Exploration Requirements - Hypothesis \# 5}

End-users require instantaneous visual responses from the lighting control interface while performing control tasks to experience its conversational property. Feedback is a well-known concept in the science of control and information theory dealing with informing end-users about what action has been done and what has been accomplished with the device, allowing them to continue with the activity (Norman, 1990 c1988, p. 27). Tidwell argues that this "success experience" provides instant gratification and confidence, which prompts end-users to keep using the device even if it gets harder later (2005, p. 11). She further argues that end-users have a naturalistic tendency of performing an incremental style of work going back and forth to see if the result is alright (p. 14). Hornecker and Bruns in their study of the "Sensoric Garden" installations that combine "multimedia with novel multi-modal interaction techniques" have observed that installations providing high control and instant multi-sensorial feedback, offering simple means of interaction with a direct and transparent mapping of action and reaction were an "interactive success" and resulted in prolonged engagement by end-users (2004). For example, a dynamic mapping between controls and its resultant effects in the living room is an essential requirement for Bruce to continue working with the interface to program the movie scene. The interface can provide an accurate visual response every time a particular lighting level or scene has been selected in the living room, to keep him informed. Interacting with interface will be fun and give him confidence when it provides an instant response such as a luminous graphic that changes luminous intensity or colour every time he dims a lighting layer. A lack of immediate response between the mapped controls and actions 
performed with the interface doesn't help as he will find it difficult to remember what action has been taken. The response can be a natural consequence of his actions, informing him about the actions performed, showing that the interface is responding and confirming navigation. Krippendorff and Butter however argue that there is little feedback at the recognition stage $(2008$, p. 360$)$.

\subsection{End-user Reliance}

Hypothesis \# 6: End-users perform the different lighting control functions quickly and effectively when the lit environment and the interface become a single entity while achieving the desired lit scenario. Krippendorff and Butter argue that reliance involves focus on what the end-users wish to accomplish with the artefact instead of attending to how it is handled; the artefact recedes into the background, is taken for granted and no longer noticed (2008, p. 361 ).

\subsubsection{Reliance Requirements - Hypothesis \# 6}

End-users require a lighting control interface that merges with the lit environment while enabling them to achieve desired lighting scenarios that suit their activities. Krippendorff and Butter argue that reliance is the stage where technology disappears from our attention, where we do not need to reflect on what something means to us, and where we can address what actually matters to us (2008, p. 361). Weiser reinforces this by arguing that the most profound technologies are those that disappear by weaving themselves into the fabric of everyday life until they are indistinguishable from it (1999, p. 3). Hallnäs and Redström comprehensively describe this change of technology's relation to endusers as a shift from "use" to "presence" (2002). Viewing interfaces from 
"use" perspective focuses on functional aspects whereas "presence" perspective touches upon broader existential definitions of design in the life of end-users. A popular example cited by Ross and Keyson for describing technology from "use" and "presence" perspectives is the mobile phone and the communication technology it offers: its functional aspects include dialling, ringing etc., while its existential definitions include feelings of social connectivity apart from the mobile phone being a means of personal expression (2007, p. 69). For example, apart from performing all the lighting control functions, Bruce would like the interface to provide an existential definition in the form of connecting with the lighting in every room of the residence. The graphical representations on the interface can show the image of the living room along with its lighting layers while he is programming the movie scene. The representations can change from living room to kitchen when he moves into the kitchen to make some popcorn for the movie and wants to turn on a light in the kitchen. He should also be able to communicate with the lighting in the living room from the kitchen while making popcorn. At a broader level of connectivity through the interface, Bruce should be able to set a desired lighting scenario in the dining room for an evening dinner at their residence while working in his office.

\subsection{End-user Attributes}

"Know thy users!" is a popular maxim in the field of interaction design. Tidwell argues that good interface design starts with an understanding of end-users: the more one knows about them and empathises with them, the more effectively one can design for them (2005, p. 3). A first step towards knowing about end-users is to identify their cognitive, perceptual-motor and emotional state such that the way they understand 
and experience interface with the lit environment can be monitored and regulated. But before classifying end-users, a more fundamental question as put forward by Tidwell is, how much effort are end-users willing to spend to learn the interface $(2005$, p. 8). For example, those rare occasions like in the living room when Bruce may use the interface every day for selecting different lighting scenarios for different occasions, motivation levels to learn it well may be high. On the other hand, Ann who only uses this interface sometimes will only learn it well enough to get by. And finally there are their friends and extended family members who will only see this interface for a few seconds. Therefore, it is important to identify whether most end-users can become intermediates to experts, or will remain perpetual novices.

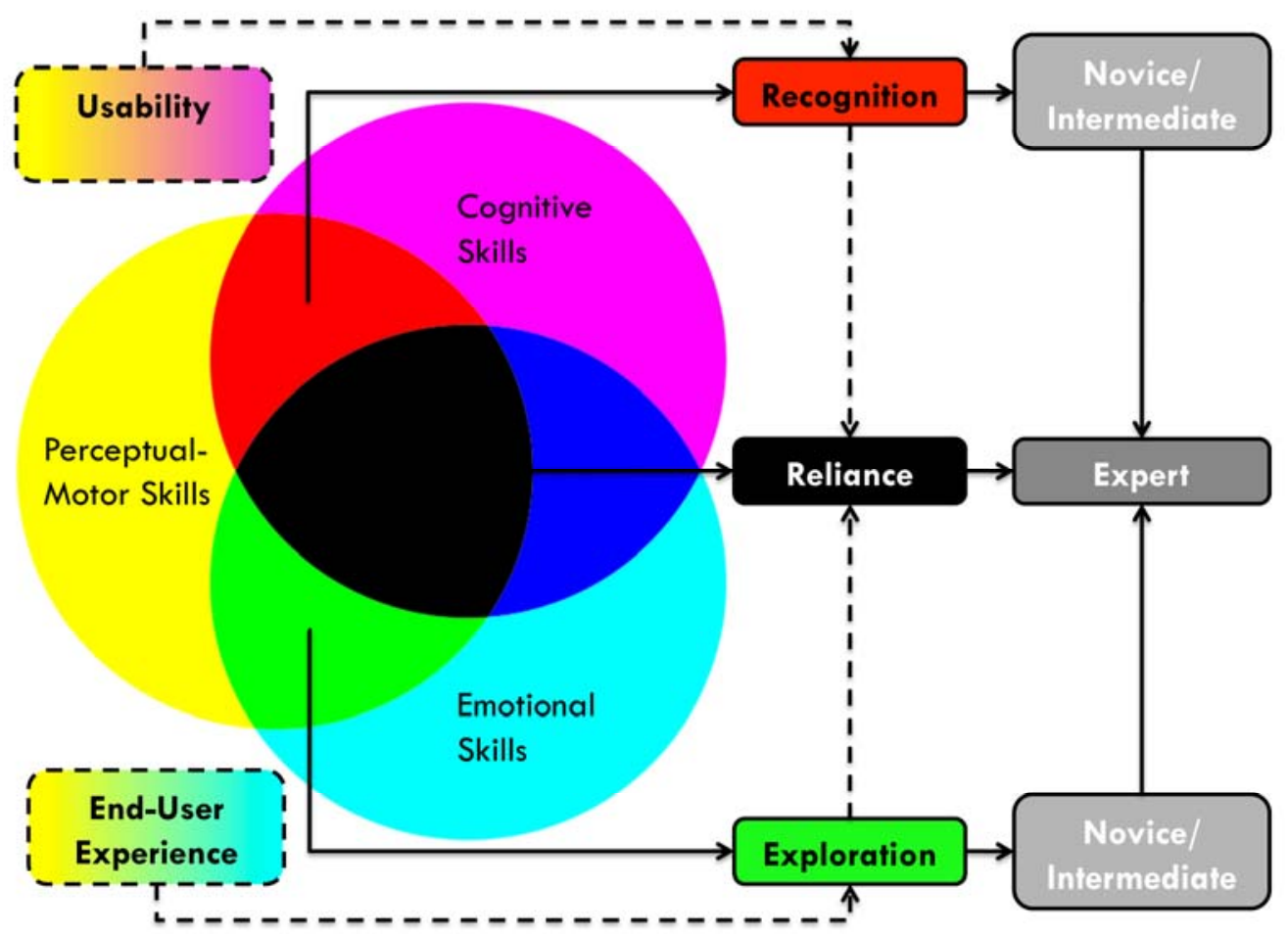

Figure 4 - Relationship between the three human skills, end-user requirements and end-user attributes 
Thomassen argues that the ideal interface design should be suitable to all end-user profiles (2003, p. 56). For example, even if Bruce, Ann, their friends and extended family members might have different and conflicting requirements, this interface should ideally be designed to meet everyone's requirements. Figure 4 describes an abstract relationship between the three human skills, end-user requirements and end-user attributes.

\subsubsection{End-user Attributes - Novice}

A novice end-user is one who has never seen or used the interface before and needs guidance on how to use it. The best guidance may be by recognising its physical design and representations, and exploring its manipulative and interactive properties to experience the resultant lighting effects. For example, Bruce and Ann's friends and family member can understand the purpose of this lighting control interface with a self-explanatory design that describes the switching, dimming and preset control functions.

\subsubsection{End-user Attributes - Intermediate}

An intermediate end-user is one who seldom uses the interface to perform lighting control tasks and will only learn it well enough to accomplish certain required tasks. A mode of enticing such an end-user is an interface that segregates the most commonly used control function keys from the rarely used ones, again by recognition and exploration. For example, Ann who only uses this interface sometimes can recognise all her most commonly used control functions keys from the given physical design and representations that will enable her to go about her tasks. 


\subsubsection{End-user Attributes - Expert}

An expert end-user is one who is skilled in using the interface and regularly uses it to perform the same lighting control tasks. It may be annoying for this end-user to work through a lot of control function keys and submenus for performing simple everyday tasks of switching, dimming, and setting or recalling lighting scenes. For example, Bruce who uses this interface everyday can have a custom set of settings labelled 'Bruce's Settings' which displays all his most commonly used control functions keys. He can select this function every time he uses the interface that will enable him to go about the everyday tasks and not worry about basic functions.

\subsection{Chapter Inference}

Chapter 3 has revealed that recognition, exploration, and reliance define a three-staged dynamic for end-user interaction and an ideal interface collectively caters to the requirements of novice, intermediate and expert end-users. By recognition end-users identify the function of the interface before it is positioned in relation to the end-users' body for exploration. By exploration end-users understand how to handle the interface and monitor the consequences of their actions to reach reliance. By reliance end-users' workflow proceed without doubts and uncertainties. Recognition and exploration are two transitional stages that cater to the novice and intermediate end-user profiles. Ideally, in order to come to what really matters and achieve reliance, end-users should not be stuck in these stages but become experts.

This chapter has also hypothesised several necessary requirements for enhancing end-user interface with personalised lighting controls. The 
usability requirements of lighting control interfaces are meant to enhance the speed and effectiveness of learning and performing the control tasks. The end-user experience requirements of lighting control interfaces are meant to enhance the pleasure and playfulness of performing the control tasks. These requirements are collectively meant to augment informationbased actions performed by end-users with lighting control interfaces. Meaningful typography and accurate iconography of lighting control functions represented on the interface enhance end-user understanding of control information. Additionally, the physical designs of interfaces along with its tactile and conversational properties enable end-users to understand its control function, as well as experience pleasure and playfulness. The affordance of a lighting control interface can ultimately provide feedback through a visual model of the natural mapping that exists between the control and its effects in the real world.

In Chapter 3, the usability and end-user experience requirements for end-user interaction with lighting control interfaces have been hypothesised. These requirements will now have to be translated into design principles for evolving key characteristics of tangible lighting control interfaces. This requires a detailed study of the existing views on tangible interaction. Chapter 4 will evolve key characteristics of tangible lighting control interfaces by translating the end-user requirements into principles for designing interfaces, before Chapter 5 uses these characteristics to develop a tool for evaluating the usability and end-user experience of lighting control interfaces. 


\section{CHAPTER FOUR}

\section{Characterising Tangible Interfaces}

\subsection{Chapter Intent}

Chapter 4 characterises tangible interfaces for attaining tangibility in end-user interface with personalised lighting controls. In Chapter 3, the three-staged requirements for end-user interface in terms of recognition, exploration, and reliance are hypothesised. Although the ideal interface is one that caters to all kinds of end-user profiles, the aim of this thesis research is to design quality lighting environments by reaching the stage of reliance. Hornecker and Buur however argue that our current understanding of human interaction with "hybrid or augmented environments" is very limited (2006, p. 437). They further argue that tangible interaction encompasses a broad scope of systems, building upon and synthesising the following three views: a data-centred view pursued in Computer Science and Human-Computer Interaction $[\mathrm{HCl}]$; an expressive-movement centred view from Industrial and Product Design; and a space-centred view from Interactive Arts and Architecture (p. 438).

The data-centred view defines Tangible user interfaces [TUls] as utilising physical representation and manipulation of digital data, offering interactive couplings of physical artefacts. The expressive-movementcentred view aims to go beyond form and appearance of products, and design the interaction itself by emphasising bodily interaction with objects. The space-centred view increasingly discusses interactive spaces 
by building installations based on spatial interaction. Therefore for enduser interface with lit environments to reach the stage of reliance through tangible interaction, reviewing each of these three different views in detail is central. The outlook is towards deriving two aspects of tangible lighting control interfaces: principles for design and key characteristics.

Literature reviews on the data-centred, expressive-movement-centred, and space-centred views on tangible interaction are done in Sections 4.2, 4.3 , and 4.4 respectively, to derive a set of synthesised principles for designing tangible lighting control interfaces. These principles can be defined as the most efficient and effective ways for making end-users recognise, explore, and rely on lighting control interfaces. The characteristics of tangible lighting control interfaces are subsequently evolved from these principles in Section 4.5. Section 4.6 then summarises Chapter 4, and draws inferences for designing interactive studies in Chapter 5.

\subsection{Tangible Interaction: Data-centred view}

The design principle derived from the data-centred view on tangible interaction is that tangible lighting control interfaces should give tangible form to control information in a lit environment by employing artefacts that perform the dual role of representation and control. Ullmer and Ishii argue that reliance on screen-based text and graphics to interact with computers has cultivated a predominantly visual paradigm of $\mathrm{HCl}(2001$, p. 579). Ramstein, Martial, Dufresne, Carignan, Chassé, and Mabilleau further argue that Graphical user interfaces [GUI] are becoming the standard in $\mathrm{HCl}$ as information is "spatially and iconically" represented, and manipulated with pointing devices, which enables sighted individuals 
to easily learn and use them (1996, p. 2). Holmquist, Schmidt, and Ullmer however, argue that the design space of $\mathrm{HCl}$ is significantly extended by moving from the virtual estate of the screen into the physical estate of the real world thereby enabling new and richer forms of interaction (2004, p. 291). They further argue that this movement from the virtual world to the physical world is achieved when interactive couplings of physical artefacts with "computationally mediated digital information" are offered by TUls (p. 292). Buur, Jensen, and Diajadiningrat argue that the core challenge in the $\mathrm{HCl}$-rooted view of TUl is how data is represented in physical objects, and how the data may be controlled through configuring these objects in the 2D or 3D space (2004, p. 186).

Ullmer and Ishii's proposed TUI example of the abacus differs markedly from the mainstream GUI as it does not make a distinction between "input devices" like keyboards and mouse as "controls," and graphical "output devices" like monitors and head-mounted displays as visual "representations" (2001, p. 579). "Urp" is another TUl example used for urban planning introduced by Underkoffler and Ishii, which is concerned with the above notions about external representations of information with physical objects and its subsequent control (1999). It is built around a workbench that allows the direct manipulation of building models to configure and control an underlying urban simulation. Urp's building models cast graphical shadows onto the workbench surface, corresponding to solar shadows at a particular time of day. The intershadowing problems can be visualised by controlling the position of the sun by turning the physical hands of a clock tool, and moving or rotating the building models, their corresponding shadows transforming accordingly. Different material properties can be bound to individual building models such as glass to cast solar reflections along with solar 
shadows. Finally, an airflow simulation can be activated with field lines graphically flowing around the building models.

Ullmer and Ishii have used Urp as an example to develop an interaction model called "MCRpd" or "model-control-representation [physical and digital]" and its four characteristics (2001, pp. 582-583). MCRpd integrates TUl's physical representation and control at a conceptual and physical level as TUI artefacts physically embody both the control pathway and a central representational [information-bearing] aspect of the interface. The following paragraphs describe how the principle of controllable representations for designing tangible lighting control interfaces has been derived from the MCRpd model and its four characteristics.

\subsubsection{The Principle of Controllable Representations}

The central characteristic of the MCRpd model lies in the coupling of physical representations to underlying digital information and computational model. The significance of this characteristic is that tangible lighting control interfaces can couple representations of lit environments to the underlying control information in these environments. For example, a builder-style interface with a realistic visual representation of the lighting layers in the living room that displays what the whole lighting looks like while Bruce learns to control it will support his style of work for achieving desired lighting scenarios.

The second characteristic is that physical representations embody mechanisms of interactive control. The significance of this characteristic is that tangible lighting control interfaces can embody mechanisms for interactive physical control where the physical movement and manipulations of the representations serve as primary means of control. 
For example, each of the realistic visual representations of the lighting layers can be physically manipulated by Bruce to perform the control tasks of navigation, selection, and grouping, switching or dimming.

The third characteristic is that physical representations are perceptually coupled to actively mediate digital representations. The significance of this characteristic is that a balance between lit environments and tangible lighting control interfaces can be achieved when the representations present dynamic information that is perceptually coupled with the lighting scenarios in these environments. For example, when Bruce decreases the luminous intensity of the ceiling cove layer in the living room, its realistic visual representation on the interface can simultaneously decrease in luminous intensity.

The fourth characteristic is that the physical state of interface artefacts partially embodies the digital state of the system. The significance of this characteristic is that the realistic representations on tangible lighting control interfaces can embody the actual state of the control system in the lit environment. For example, the position of the dimming control handle can determine the accurate level of luminous intensity selected by Bruce for the ceiling cove layer in the living room.

\subsection{Tangible Interaction: Expressive-Movement- centred view}

The design principle derived from the expressive-movement-centred view on tangible interaction is that tangible lighting control interfaces should appeal to the visual and tactile senses by employing artefacts that afford the right actions and expressively respond to those actions. Buur, 
Jensen, and Diajadiningrat argue that the data-centred view on TUI is limited due to two reasons: firstly, it assumes that computers are foremost information processing devices, while in reality products with embedded microcontrollers such as washing machines monitor and control things in the real world; secondly, it assumes that interaction is primarily a cognitive activity, while in reality people's activities are very much physical (2004, p. 186).

Djajadiningrat, Wensveen, Frens, and Overbeeke argue that most electronic products with decision trees and menu structure actually feel very "PC-like" in interaction style, only worse, because of their lack of screen real estate and full-sized input devices (2004, p. 294). They further argue that in addition to a data-centre view, it is also posssible to take a perceptual-motor-centred view on tangible interaction as physical objects provide "rich opportunities for differentiation in appearance and action" (p. 294). Diajadiningrat, Overbeeke, and Wensveen have observed that this view emphasises a respect for perceptual and bodily skills, what end-users can perceive and what they can do with their body: their affectivities (2002, p. 288). Buur, Jensen, and Diajadiningrat see "rich actions" an essential approach to tangible interaction, when human motor skills become crucial for interaction with technology requiring endusers to build bodily skills over time (2004, p. 187). Hornecker and Buur argue that the design of such products takes account of embodied skills, focuses on expressive movement and "rich" interaction with "strong specific" products tailored to a domain (2006, p. 438).

In Diajadiningrat, Overbeeke, and Wensveen's opinion, "good TUI design" is made of two criteria that appeal to all our senses and fit our bodily skills: "feedforward and inherent feedback" (2002, p. 291). Feedforward informs end-users about what the results of their actions will 
be (p. 286). Although inviting the appropriate action is a prerequisite for feedforward, it is not sufficient, as the product also needs to communicate what end-users can expect. Feedback informs end-users about the actions performed, showing that the product is responding and confirming navigation; inherent feedback is an experience end-users have as a natural consequence of their actions (pp. 289-291). The following paragraphs describe how the principle of expressive reciprocations for designing tangible lighting control interfaces has been derived from the two criteria of feedforward and inherent feedback.

\subsubsection{The Principle of Expressive Reciprocations}

Feedforward increasingly discusses the operation of a control having directly perceivable consequences in the real world with natural mapping offering a solution for room lighting: the way the product components are meaningfully laid out in space can help end-users understand their purpose in the real world. Additionally, the way a control looks and the action it requires express something about the control's purpose. The significance of this criterion is that representations on tangible lighting control interfaces can naturally and meaningfully map the lighting layers of the lit environments so that their control operations have directly perceivable consequences. For example, the realistic representations of the lighting layers in the living room can enable Bruce to get a preview of his desired lighting scenario; the design of the control handle can enable him to understand its function and the right actions required for operation.

Inherent feedback requires unity of location, where the action of the enduser and the feedback of the product occur in the same location. The significance of this criterion is that tangible lighting control interfaces can provide a perceptual response in the same location where the end-users 
perform the action. For example, when Bruce performs the control action of dimming the luminous intensity of the ceiling cove in the living room, its realistic visual representation on the interface can also change in luminous intensity to strengthen the inherency of the visual response as input and output occur in the same spot.

Inherent feedback requires unity of direction, where the direction of the product's feedback is the same as the action of the end-user. The significance of this criterion is that tangible lighting control interfaces can provide a directionally similar perceptual response as the action of the end-users. For example, if the decrease in luminous intensity is conceivably different from Bruce's 'decrease' action, such a deviation can weaken the inherency of the visual response.

Inherent feedback requires unity of time, where the product's feedback and the end-users' action coincide in time. The significance of this criterion is that the visual response provided by tangible lighting control interfaces can be coupled with end-users' actions. For example, if there is too much of a delay between Bruce's action of decreasing the luminous intensity of the ceiling cove and the visual response provided by its realistic representations on the interface, they can no longer be seen as related.

\subsection{Tangible Interaction: Space-centred view}

The design principle derived from the space-centred view on tangible interaction is that tangible lighting control interfaces should be contextually situated in lit environments thereby making actual experiences pivotal in the design process. Ross and Keyson argue that 
the context in which the expressive tangible device will be used should be taken into account before designing the physical properties of the device (2007, p. 70). Krippendorff and Butter's description about relationships between artefacts and their contexts being rendered sensible and coherent by attributing meaning to artefacts provides a direction for designing the contextual situation of interfaces (2008, p. 362).

Ross and Keyson further argue that as the complex nature of experiential factors influencing interaction may be difficult for designers to perceive, physical models and real-life "experiential" testing in context is crucial to learn how design decisions may influence end-users' interaction experience; however they also argue that every mock-up in the design process does not have to be fully functional (2007, p. 71). Hornecker's description about responsive environments in interactive arts and architecture that rely on systems physically embedded in real spaces, combining real space and real objects with digital displays offering opportunities for interacting with tangible devices provides a direction for designing such real-life mock-ups (2006a, p. 22). Bongers' idea of an "Interactivated Space" is to create a responsive environment that interacts with the people that are in it by sensing their activity and reacting through haptic, iconic, kinetic or sonic displays (2002, p. 1).

Rubidge and MacDonald discuss the importance of legibility of the interface and the use of the sensory systems as the main interface with such responsive environments (2004, p. 247). The computer system in such responsive environments receives input from the real world through sensors, and addresses the senses of end-users through output devices such as actuators and displays, which enables the system to interact with end-users in a multimodal way. The communication channels between humans and computer systems that reflect the human senses and actions 
are in the form of visual [texts, colours, moving images], auditory [speech, music] and tactile [touch] modalities. The following paragraphs describe how the principle of contextual experiences for designing tangible lighting control interfaces has been derived from the concept of embedded systems that are both meaningful and physical in an interactivated space.

\subsubsection{The Principle of Contextual Experiences}

The context of the expressive tangible device and its relationship to other objects in the environment should be taken into account. The significance of this criterion is that tangible lighting control interfaces can meaningfully and physically become a part of the lit environment in terms of look and feel as well as end-users' daily activities and rituals. For example, Bruce's interface can look like and also perform the activities of a mobile phone such as making phone calls or listening to music while taking a stroll in the park.

The expressive tangible device should undergo a process of real-life experiential testing in mock-up environments. The significance of this criterion is that the mock-up can be in the form of an interactivated space where the computer system receives input from the tangible lighting control interface about the desired lighting scenario and displays visual images of the lit environment on a screen. For example, before installing the lighting control system and its interface in Bruce's residence, a mockup immersive and responsive environment can be built at the lighting control manufacturer's outlet in the city where visual displays of lit interiors are projected onto a screen. The mobile phone can be programmed to communicate with a computer via wireless channels, which in turn projects different lighting scenarios on the screen when operated. This way Bruce can get a first-hand experience of the interactive lit 
environment even before installing it in his residence, while the lighting design team can get a better understanding of Bruce's needs and expectations from the lighting control system and its interface.

\subsection{Tangible Interfaces: Key characteristics}

Richly represented information describing the interactive lit environment that in turn richly responds to end-users' actions are identified as the two key characteristics of tangible lighting control interfaces. These characteristics are in essence attributive concepts, which have been evolved from the synthesised principles for designing tangible lighting control interfaces and the end-user requirements for interface with personalised lighting controls in Chapter 3. They are trends distinguishing tangible lighting control interfaces from other interfaces, but are not directly related to the process involved in designing interfaces.

\subsubsection{Key Characteristic \# 1 - [Rich] Representation}

Richness of representation is the strength and salience of the tangible lighting control interface to describe its intended control function and operation. It can be defined in terms of the meaningfulness and longlasting impression created by the physical designs and external representations in the minds of end-users for learning and remembering how to use it. This characteristic will provide rich clues for end-users to quickly and effectively learn its intended function and operation. Richness of representation can have three broad dimensions.

The first dimension is the content and format of information embodied in the physical designs and external representations of interfaces. For example, the appearance and design of the interface itself can permit 
Ann to learn its functional and operational use of dimming the lighting inside the bedroom.

The second dimension is the accuracy of information provided by the physical designs and external representation of interfaces. For example, the representations of the illumination levels can enable Ann to accurately dim the lighting in the bedroom to her desired illumination level.

The third dimension is the timeliness of information provided by the physical designs and external representations of interfaces. For example, the appearance and design of the interface can enable Ann to quickly learn to dim the lighting in the bedroom.

\subsubsection{Key Characteristic \# 2 - [Rich] Reciprocation}

Richness of reciprocation is the ability of the tangible lighting control interface to invite end-users to interact by providing sensory pleasure and playfulness. It can be defined in terms of the 'conversational' type of interaction provided by the interface, giving constant informative and looped responses, enabling end-users to understand about their actions and proceed in small experimental steps. This characteristic will enable end-users to easily grab and feel its movable parts as well as understand the relation between their actions and its effects to experience its rich interactive property. Richness of reciprocation can have two broad dimensions.

The first dimension is the tactile response received from the interface by having haptic contact and feeling material properties. For example, the interface control handle that has a large surface area and texture can enable Ann to experience its tactile property. 
The second dimension is the visual response received from the interface every time end-users navigate, select and group their desired layers of lighting as well as switch or dim them to achieve their desired lighting scenarios. For example, a luminous graphic that provides Ann with a clear link between what she does with the interface and what happens to the lighting in the bedroom can enable her to experience its conversational property.

\subsection{Chapter Inference}

Chapter 4 has derived a set of synthesised principles for designing tangible lighting control interfaces from the three dominant views on tangible interaction. The principle of controllable representations describes how interfaces can couple realistic representations of lit environments with direct manipulable controls. The principle of expressive reciprocation describes how naturally mapped realistic representations of lit environments can provide instantaneous responses. The principle of contextual experiences describes how situational contexts and immersive mock-ups can enhance the process of designing interfaces. These principles assert that there are techniques, methods and processes that are more effective than the current techniques at inducing end-user interaction with lit environments and lighting control systems. They determine what end-users should see and do while using personalised lighting control interfaces, and provide specific guidelines for enhancing their usability and end-user experience.

These principles along with end-user requirements for recognition, exploration and reliance for Chapter 3 have been used to evolve two key characteristics of tangible lighting control interfaces: [Rich] 
Representation and [Rich] Reciprocation. The first characteristic describes how physical designs and external representations can provide rich information, thereby enhancing the understanding of these interfaces. The second characteristic describes how physical designs and external representations can provide rich sensory experiences, thereby enhancing pleasure and playfulness in using these interfaces. Lighting control interfaces that reflect these characteristics will invariably meet the usability and end-user experience goals for designing quality lighting environments.

In Chapter 4, the principles for designing tangible lighting control interfaces have been derived and their key characteristics have been evolved. These characteristics will now have to be transformed into tools for evaluating the usability and end-user experience of lighting control interfaces. These tools will essentially embody a causal relationship between end-users' behavioural responses that are dependent on the type of lighting control interfaces used by end-users. Chapter 5 will design interactive studies that scientifically analyses and documents enduser behaviour with lighting control interfaces. These studies will be used to evaluate interfaces as well as provide new ideas for designing better existing and future tangible interfaces. 


\section{CHAPTER FIVE}

\section{Designing Interactive Studies}

\subsection{Chapter Intent}

Chapter 5 designs interactive studies to enable end-users use the key characteristics as tools for evaluating the usability and end-user experience of lighting control interfaces. In Chapter 4, two attributive concepts that characterise tangible lighting control interfaces have been evolved. However the overall aim of this thesis research is also to develop a tool that enables end-user evaluation of usability and enduser experience with lighting control interfaces. This involves documentation of observable and variable end-user responses towards these interfaces. Levine and Parkinson state that events and phenomena that are potentially variable, and in which observers have some interest are called variables (1994, p. 9). Brewer defines the variables that are manipulated for obtaining observers' responses as the "cause" or "independent variables" and the responses itself as the "effect" or "dependent variables" (2000, pp. 3-4). The key characteristics should therefore embody a 'cause-effect' relationship where the lighting control interfaces become the independent variables and the end-users' behavioural responses become the dependent variables.

Studies on behavioural research employ survey research as a strategy to study scientifically this causal relationship between the independent and dependent variables. Levine and Parkinson further argue that scientific 
investigational settings such as these require operational definitions of all the involved variables so as to accurately measure the causal relationship between them (1994, p. 25). Research can be described and evaluated in terms of three types of validity, each giving a different perspective on any particular investigation: construct validity, internal validity and external validity (Cozby, 2001, p. 72). There is never a perfect definition of a variable because they can be measured and manipulated in a variety of ways. Construct validity refers to the adequacy of the operational definitions of variables in reflecting the true theoretical meaning of the variables. The success of a research project is gauged by the ability to draw strong inferences about the cause-effect relationship between the variables. Internal validity refers to the ability to draw strong conclusions about the causal relationships from the gathered data. Finally, the results of the data should be replicable with different participants in different settings. External validity is the extent to which the results of the study can be generalised to other populations and settings.

Proper scientific behavioural research methods of observation and documentation have to be used in these studies. The methods should enable participants to provide information about themselves - their attitudes and beliefs about the lighting control interfaces used in the studies, and opinions about their most desirable interfaces. The methods used should require minimum effort from the participants and incorporate suitable measures for quality control. The probability of achieving definite results is maximised when all extraneous variables are neutralised and kept constant. Experimental control and randomisation eliminate the influence of many extraneous variables (Cozby, 2001, p. 66). The outlook is towards gathering ample evidence to support the arguments put forward by this thesis research. 
Literature reviews are done in Section 5.2 to identify scientific survey research methods that can be used for observing end-user behaviour with lighting control interfaces. Section 5.3 underlines the principal objectives for designing these interactive studies. Section 5.4 introduces the nature of the independent and dependent variables used in these studies, and operationally defines them. Section 5.5 describes a simple procedure used for data collection and analysis with pen-and-paper instruments. Section 5.6 reviews literature to describe the necessary quality control measures that need to be taken against the effects of any extraneous variables. Section 5.7 then summarises Chapter 5, and draws inferences for executing the interactive studies in Chapters 6 and 7.

\subsection{The Methods: Experimental and Non-experimental}

This thesis research employs a strategy that combines experimental and non-experimental methods to gather, evaluate, and report information on end-users. Cozby underlines two general methods for studying behaviour: the experimental method and the non-experimental method (2001, p. 60). The experimental method is designed for confirmatory purposes of testing hypotheses by gathering quantitative data. The nonexperimental method is designed for exploratory purposes of generating and/or verifying hypotheses by gathering qualitative data. The difference between the two methods has important implications, as no single method is perfect or definitive for studying end-user behaviour. Therefore quantitative data is obtained through experimental methods, and qualitative data is obtained through non-experimental methods. Robson explains that when different kinds of data are collected from a study, it is regarded as a research strategy rather than a research method (2002). 


\subsubsection{Experimental Method - Quantitative}

The experimental method involves introduction and manipulation of the different types of lighting control interfaces with the intention of observing and measuring the different operational definitions of the key characteristics. Empirical studies and controlled experiments in labs offer precision for answering specific questions about comparative effectiveness of design options, identification and diagnosis of problems, or to validate that the design has satisfied its goals (Butler, 1996, p. 70). Testable hypotheses concerning the relationship between lighting control interfaces and end-user responses are made at the start of the study. The quantitative data gathered from this method are coded and analysed using statistical scores meant to either support or refute these hypotheses, thereby testing the arguments proposed by this thesis research.

The design of the experimental method used in this survey research can be classified under "Repeated Measures Design" as it requires all participants to repeatedly measure the different dependent variables after receiving each level of the independent variable (Wendorf, 1997). Normally in a repeated measures design, two sets of scores represent measures of the same thing, and exactly the same test is given under both conditions to all participants (Hayes, Gee, Price, Cooksey, \& Patrech). The advantage of using the repeated measures design is that the experiment can be conducted with an unusually low number of participants, which increases its efficiency and sensitivity by keeping a low variability in individual differences (Minke, 1997). 


\subsubsection{Non-experimental Method - Qualitative}

The non-experimental method involves gathering end-user opinions about their desired interfaces in their own words. Nielsen and Mack argue that in heuristic evaluation [HE] of interfaces it is not sufficient for the expert inspector to say they do not like something; they should also explain why they do not like it with references to those usability principles that were violated (c1994, p. 31). Butler argues that the advantages of these "think aloud" techniques is that they allow designers some direct access to what end-users are thinking and what problems they are aware of having (1996, p. 70). In this method, observations of end-user behaviour with lighting control interfaces are done by asking end-users to describe their needs, hopes and aspirations about desired interfaces through continuous prose, listed answers, or conceptual sketches. The thesis research requires end-user ideas, hopes and aspiration about their desired lighting control interfaces to arrive at general qualities required for improving existing interfaces as well as designing tangible interfaces. The gathered qualitative data provides conceptual ideas for designing better existing and future tangible interfaces.

Employing a suitable coding system to categorise, analyse and use the gathered qualitative data for developing new designs is essential. One method of coding qualitative data is by systematic observation, where careful observation of one or more specific behaviours in a particular setting is recorded. The specific behaviours that interest this thesis research are better characteristics of existing interfaces and the desire for particular characteristics of tangible interfaces. This method of coding can also be used for quantifying the qualitative data while providing vital leads for the design development of future tangible interfaces. 


\subsection{The Objective: Study end-user behaviour}

The principal objective of designing these interactive studies is to attain a complete and methodical understanding of end-users' behaviour with lighting control interfaces. Defining end-user population is essential in determining who will be at the centre of the design process (Butler, 1996, p. 64). Additionally, as studying an entire population is an enormous undertaking, samples are selected from this target population. The overall design of the interactive studies for observing and documenting end-user behaviour can be classified under the category of "Crosssectional Design" as the samples are tested only once to gather and compare participant responses (Giles, 2002, p. 100). The process involves studying end-user behaviour with existing and tangible interfaces in two parts: an Existing interface [El] study and a Tangible interface $[\mathrm{TI}]$ study.

These studies are designed to involve potential end-users in a reactionary role to evaluate lighting control interfaces, and a participatory role to provide ideas about their most desirable interfaces. Responses obtained from the El study were combined with the synthesised principles for designing tangible lighting control interfaces from Chapter 4, to evolve prototypes of future tangible interfaces. These prototypes were tested in the TI study. The results obtained from the El study were also used to fine-tune the overall testing pattern and procedure for the TI study. The repeated measures design of the interactive studies was ideally suited for the TI study as all participants performed the same tasks with two prototypes of interfaces, an existing and a future tangible, in a single test. However, in the El study all the participants performed the same tasks with more than two types of existing interfaces in a single test. 


\subsubsection{Identify End-users - Sampling population}

Identification of a target population for the studies and then sampling them is the fundamental step of behavioural research. This involves sampling participants from a population of interest so that a precise estimation of the characteristics of the population as a whole can be made. Representative samples of end-users are needed to gather knowledge about their application domains and to support empirical user testing (Butler, 1996, p. 64). The interactive studies specifically required participants in a participatory role, who could provide cogent descriptions of end-user actions while performing lighting control tasks, which in turn is aimed at designing better existing and future tangible interfaces. Jensen, Buur, and Diajadiningrat have found it valuable to work in cooperation with design students as informants to develop design methods that give "primacy of place to user actions" $(2005$, p. 10).

A decision about the exact sample size also had to be made for conducting the studies. Researchers have reported that for a minimum sample size of greater than or equal to thirty, the population tends to be normally distributed (Salkind, 2007, p. 153; Sekaran, 2000, p. 296). Additionally, by ensuring that all participants in the tests were selected in a random fashion and given an identical treatment, the influence of any form of individual characteristics could be eliminated. Research reveals that age and gender have a certain amount of impact on individual interface preferences (Balakrishnan \& Yeow, 2010; Cyr, Head, \& Ivanov, 2005). However, considering the fact that overall end-user responses towards interfaces are more important than individual preferences, exploratory analyses of age- and gender-based preferences will not be done for these interactive studies. Additionally, the sample size was not considered significant enough to disturb the overall results. Therefore, a simple random sampling method was used wherein lists of students and 
staff members from Victoria University of Wellington, New Zealand were made. At least thirty participants for each study were chosen at random from this list to form the samples. Special care was taken to include a good cross-section of participants from different ages for arriving at a comprehensive set of results. A good cross-section of participants also reduces all other forms of interviewer biases involved in conducting behavioural research with human participants.

\subsubsection{Interactive Study \# 1 - Existing interfaces}

The El study evaluates and compares the usability and end-user experience of existing interfaces against each other. There are three reasons for enabling participants evaluate existing interfaces. The first and most obvious reason is to involve participants in a reactionary role to obtain their responses with the different generic types of existing interfaces. Participants were asked to perform a set of lighting control tasks with these interfaces and evaluate them with the operational definitions of the key characteristics. The second and subtler reason is to involve participants in a participatory role to obtain their needs and aspirations about the features of the most desirable interfaces. The act of making participants perform a set of lighting control tasks with existing interfaces encouraged their creative thought process for evolving conceptual designs of desirable interfaces. The third and completely discreet reason is to test the strength and salience of the key characteristics as tools for enabling participants evaluate interfaces. The overall success of this research is partially dependent on the ability of the key characteristics to embody end-user responses towards interfaces for lighting control. Results of this study will provide evidence to measure the strength and salience of these characteristics as evaluation tools. 
However, participants of the El study were presented with lighting control tasks of controlling luminous intensity or colour and recalling preset lighting scenes only. These tasks require less cognitive processing in comparison to programming and setting lighting scenes. This in turn reduces the cognitive load of doing too many complicated tasks while fulfilling the three reasons of this study. Additionally, the interfaces required for performing these control tasks namely pushbutton, rotary and slide, could be easily built and tested under experimental conditions.

\subsubsection{Interactive Study \# 2 - Tangible interfaces}

The TI study evaluates and compares the usability and end-user experience of tangible lighting control interfaces against existing interfaces. Based on participants' responses obtained from the El study, a prototype of a tangible interface was built and tested to replicate the lighting control functions and operations of an existing interface. Participants were asked to perform a set of lighting control tasks with each of these interfaces and evaluate them with the operational definitions of the key characteristics. The lighting control tasks performed by participants in this study primarily include preset control functions of programming, setting and recalling scenes by navigation, selection and grouping of different lighting layers, along with switching and dimming. These tasks are of a more complex nature in comparison to the tasks of the El study.

\subsection{The Variables: Independent and Dependent}

The different variables being measured in a particular study have to be operationally defined in order to manipulate and measure them. Conceptual variables should be turned into a set of operations with 
specific instructions, events and stimuli before presenting them to the research participants (Cozby, 2001, p. 146). Levine and Parkinson describe operational definition as one that clearly specifies how the levels of an independent variable are to be defined or manipulated, or how scores of a dependent variable are to be measured, in a particular study (1994, p. 25). The word operational refers to the fact that the precise operations are specified for identifying differences along the dimensions of both the independent and dependent variables.

\subsubsection{Independent Variables - Interfaces}

The different types of interfaces introduced in the interactive studies embody the different manipulations of the independent variables. Levine and Parkinson use the term experimental operational definition to define the manipulations involved in the independent variable, which are specified in a way that permits other experimenters to duplicate these manipulations (1994, p. 25). Cozby has identified two types of manipulations used in behavioural research: straightforward and staged (2001, pp. 146-148). In straightforward manipulations, the stimulus presentation of the manipulations is relatively simple and upfront, normally using visual materials. However, staged manipulations are used when it becomes necessary to simulate some situation that occurs in the real world so as to successfully manipulate the independent variable. The type of manipulations used has important implications on the types of interfaces that are presented to participants in the studies. In other words, actual physical interfaces were used in tests for which authentic lit environments could be easily built, whereas simulated lit environments controlled by virtual interfaces were used for all other tests. 


\subsubsection{Interfaces - Physical}

Simple tests involving control of luminous intensity and colour do not require any form of simulated lit environments as the actual luminaire can be used to depict changes in intensity or colour. The three generic types of existing manual interfaces or manipulations used in the study are pushbutton, rotary and slide. Although the three types of interfaces are readily available in the market for manually controlling luminous intensity, there is no available technology for the manual control of luminous colours with rotating and sliding actions. Therefore, appropriate equipment was developed to custom control luminous colours using pushbutton, rotary and slide interfaces.

\subsubsection{Interfaces - Virtual}

Tests involving navigation, selections, grouping, switching and dimming of different lighting layers to program and recall preset lighting scenes are too complex and costly to create in the real world for experimental settings. Therefore, these complex tests were simulated using available expertise in computer hardware and software technology. The programming and recall of preset lighting scenes is performed with screen-based virtual interfaces. Cozby argues that although simulated laboratory experiments permit relatively unambiguous inferences, the high degree of control and the laboratory setting may sometimes create an artificial atmosphere that limits either the questions that can be addressed or the generality of the results (2001, p. 69). However, Cozby refers to Anderson, Lindsay, and Bushman's study and further argues that experiments conducted both in laboratory and field settings usually yield similar results (1999). 


\subsubsection{Dependent Variables - Key characteristics}

The two key characteristics of tangible lighting control interfaces that embody end-users' responses towards interfaces are the dependent variables. These characteristics are abstract concepts that already exist in the participants and the researcher does not control them. Each of these concepts should represent a general class within which specific instances called levels or values will vary (Cozby, 2001, p. 56). Empirical evaluation is facilitated when the basis for classification or precisely how the measurements are made is specified, and translated into specific operations or techniques so that these abstract concepts can be easily scaled and measured. Levine and Parkinson use the term measured operational definition to define all subject variables, whether or not they are dependent variables simply by specification of the means of measurement and specification of criteria used for classification (1994, p. 25).

Richness of representation is a key characteristic that attributes information represented on lighting control interfaces to end-users' learning the use of these interfaces. Doll and Torkzadeh have developed an instrument to measure end-user satisfaction with computers that merges ease of use with four information product items namely content, format, accuracy, and timeliness (1988, p. 259). The operational definitions of the first key characteristic dealing with information richness of interfaces are based on these four items. Richness of reciprocation is a key characteristic that attributes tactile and visual responses received from lighting control interfaces to end-users' pleasure and playfulness while using these interfaces. The operational definitions of the second key characteristic are based on the 'quantity' of tactile and visual responses received. Finally, the evaluation tool attempts to merge the concept of ease of use with the two key characteristics. 


\subsubsection{Key Characteristic \# 1 - [Rich] Representation}

Richness of representation can be measured in terms of the information content and format of lighting control interfaces. End-users should be able to effectively learn the control function of the interface by looking at its physical design and/or external representations. The design and/or representations can appear, or not appear like an interface for performing the intended control function. Therefore the measured operational definition of the content and format of interfaces can be defined as Appearance. The end-user behavioural responses towards the content and format of information represented on interfaces can have two operational levels: "Looks like," and "Does not look like."

Richness of representation can be measured in terms of the information accuracy of lighting control interfaces. End-users should be able to effectively select their desired lighting scenario, or repeat an exact previous selection from the range of lighting scenarios available on the interface. The interface can be accurate, sometimes accurate and sometimes not, or inaccurate for making the desired selection. Therefore the measured operational definition of the accuracy of interfaces can be defined as Accuracy. The end-user behavioural responses towards the accuracy of making and repeating selections using interfaces can have three operational levels: "Accurate," "Sometimes accurate, sometimes not," and "Inaccurate."

Richness of representation can be measured in terms of the timeliness of information provided by lighting control interfaces. End-users should be able to quickly learn the control function of the interface by looking at its physical design and/or external representations. The learning process can take very little time, some time, or a lot of time depending upon the design and/or representations. Therefore, the measured operational 
definition of the timeliness of information provided by interfaces can be defined as Learning Speed. The end-user behavioural responses towards the timeliness of information provided by interfaces can have three operational levels: "Takes very little time," "Takes some time," and "Takes a lot of time."

\subsubsection{Key Characteristic \# 2 - [Rich] Reciprocation}

Richness of reciprocation can be measured in terms of the tactile responses provided by lighting control interfaces. End-users should be able to grab and feel the movable parts of the interface in order to experience pleasure and playfulness. The interface can either be easy to grab or hard to grab depending upon the surface shape and size of its physical design. Therefore, the measured operational definition of the tactile response of interfaces can be defined as Grabbability. The enduser behavioural responses towards the tactile quality of interfaces can have two operational levels: "Easy to grab," and "Hard to grab."

Richness of reciprocation can be measured in terms of the visual responses provided by lighting control interfaces. End-users should achieve a conversational style of interaction by proceeding in small experimental steps with noticeable visual responses in order to experience pleasure and playfulness. The interface can provide an immediate response, some response or no response at all. Therefore, the measured operational definition of the visual response of interfaces can be defined as Responsiveness. The end-user behavioural responses towards the conversational quality of interfaces can have three operational levels: "Very responsive," "Somewhat responsive," and "Not responsive at all." 


\subsubsection{Merged Concept - [Ease] Use}

Ease of use can be measured in terms of the user-friendliness of lighting control interfaces. Novice end-users should be able to easily use the interface for the first time, whereas expert end-users should be able to use it every time without being annoyed. The interface can be very easy, easy, difficult or very difficult to use. Therefore, the measured operational definition of the user-friendliness of interfaces can be defined as Ease of Use. The end-user behavioural responses towards the ease of using interfaces can have four operational levels: "Very easy to use," "Easy to use," "Difficult to use," and "Very difficult to use."

\subsection{The Procedure: Questionnaires}

Questionnaires provide the opportunity to present questions in a comprehensible written format where participants can easily write their answers. The underlying assumption at the heart of the use of questionnaires is that participants are willing and able to provide truthful and accurate answers (Cozby, 2001, p. 105). Additionally, when endusers are involved in the evaluation process, expressing the criteria in a suitable format and language is especially important since they are unlikely to understand jargon and specialist notations. Moore, Carter, and Slater reinforce this by reporting that questionnaire formats based on rating scales are suited to field-based lighting research due to their reliability, ease of administration and the ease with which subsequent statistical analysis may be undertaken (2004, p. 133). Therefore, a questionnaire was designed where the different variables were presented in simplified notations. Dr. Jenny Neale from the School of Social and Cultural Studies at Victoria University of Wellington offered her expert advise in designing a preliminary questionnaire. The 
preliminary questionnaire was then circulated amongst students of the post-graduate writing group for further comments and refinement on readability and understanding of the language.

The questionnaire follows a general pen-and-paper format consisting of different questions to gather different kinds of data: closed-response for quantitative data and open-response for qualitative data. The questions are tied to the overall research aims of this project. Complete anonymity of the participant was maintained as all completed questionnaires were coded and no identifying information about the participant was asked. The results obtained from these questionnaires were coded and analysed. Analysis has two objectives: firstly, to understand the situation as-is and secondly, to produce requirements for improvement (Butler, 1996, p. 64).

\subsubsection{Type of Questions}

This thesis research requires quantitative data to confirm the hypothetical predictions, and qualitative data in the form of design ideas for developing future designs. The two types of questions that can be used for obtaining quantitative and qualitative data are called Closed-ended and Open-ended questions respectively. Closed-ended questions are structured approaches that allow participants to choose from a finite set of predetermined answers in terms of reliable rating scales to achieve quantitative data for statistical analysis. Moore, Cater, and Slater describe the two most popular rating scales used in lighting research as the Likert scale and semantic differential scale (2002b, p. 208). Participants can choose from these answers, which can then be easily coded and standardised, as the response alternatives are the same for everyone. For example, the ease of use consisting of four levels of operation on a scale of responses has a finite set of four predetermined 
answers to choose from: "Very difficult to use," "Difficult to use," "Easy to use," and "Very easy to use."

Open-ended questions allow participants to elaborate their own design concepts and ideas in the form of continuous prose, listed answers or conceptual sketches. These questions are more useful for collecting ideas and opinions as they provide participants with the freedom to describe hopes and aspirations in any preferred manner. For example, participants were requested to explain their needs and desires in the form of descriptive words or conceptual sketches defining better lighting control interfaces.

\subsubsection{Organisation and Layout}

The introduction to the questionnaires used was written to be enticing. It also clearly states the purpose of this research. The first few questions are general, non-identifying, and simple questions relating to the participants' awareness and past experiences with lighting control interfaces. These questions are meant to capture participants' attention, encourage active participation, and provide a general idea on answering questions. Most of these questions do not have any bearing on the overall analysis of the survey results. However, all questions addressing similar themes or types of control tasks are grouped together under similar headings. Open-ended questions inviting design ideas and feedback in the form of comments and suggestions are provided in the latter part of each experiment or questionnaire.

All personal, private, and sensitive questions relating to participant demographics are asked at the end of the questionnaire. Research shows that potential survey participants are most sensitive to personal questions and may be unwilling to participate in a survey if they are asked such 
sensitive questions at the beginning of the questionnaire (Montenegro, 2004). Participants' refusal to participate can adversely affect the survey research, as a new set of participants will have to be sampled causing much inconvenience and delays. Therefore, such demographic questions are asked at the end, after participants have answered all the other relevant questions. Sample questionnaires used in the El and TI studies can be viewed in Appendices 1 and 2 respectively.

\subsubsection{Coding and Analysis System}

The quantitative data was coded and measured using ordinal and nominal scales. All response variables representing different operational levels of the dependent variables are in principle polar adjectives that occupy a space on a meaningful continuum. Ordinal scales allow participants to rank order the operational levels of the dependant variables that are being studied. Nominal scales have categories or groups that simply differ from one another, instead of numerical or quantitative properties, which allow participants to categorise the variables. The two basic types of scales used in this survey research for rank ordering and categorising the participant responses respectively are called categorical ordinal scales and categorical nominal scales.

The categorical ordinal scale used in this survey research for rank ordering responses is a hybrid of the Likert and the semantic differential scale. Moore, Carter, and Slater, have put forward three reasons for using a hybrid instead of using these scales individually (2002b, p. 208). Firstly, Likert scales require participants to agree or disagree with a statement, a method of response that has been criticised due to the emotive nature of "agree-disagree." Secondly, the use of multiple responses to often fairly loosely defined stimuli in semantic differential scales is found unnecessary for projects which have clearly defined 
responses of interest and the stimuli involved. Thirdly, strict semantic differential scales are not suitable for collection of wide range of data, as they tend to bore participants leading to unconsidered or null responses. Therefore the question format of the Likert scale, with a clear definition of the response stimulus and a single response category was retained. Additionally, single semantic differential rating scales were adopted as their wording can be applied unemotionally, response definition can be clearly defined and the scales tend to be used more linearly. For example, the ease of use consisting of four levels of operation on a hybrid scale of responses has four values: "Very difficult to use - 1," "Difficult to use - 2," "Easy to use - 3," and "Very easy to use - 4." Personal data relating to participants' previous experience with lighting control interfaces and demographics that require participants to select from a given set of options use categorical nominal scales. For example, the gender of participants has two categories to choose from: "Male" and "Female."

This thesis research uses inferential statistics to draw inferences from the quantitative data. All forms of inferential statistics begin with statements about the null and research hypotheses. The research hypothesis in this case is basically the set of testable hypotheses made at the start of each study. The null hypothesis on the other hand simply states that the independent variable has no effect on end-user responses and the observed difference is due to random error. Cozby explains the logic of the null hypothesis is that if it is determined to be incorrect, then it can be accepted that the research hypothesis is correct $(2001$, p. 218). So as to precisely accept the probability of the research hypothesis, the null hypothesis has to be rejected. Therefore when there is a very low probability that the obtained results are due to random error and the null hypothesis can be confidently rejected, the results are said to be 
statistically significant. In quantitative terms, the probability required for significance is called the alpha level or $p$-value.

The outcome of a study is considered significant when there is $5 \%$ or less probability of obtaining the results or $p<0.05$ (Salkind, 2007, p. 181). Additionally, the statistical analysis of a study conducted by Bischof, Conradi, Lachenmaier, Linde, Meier, Pötzl and André shows that tests resulting in $p<0.001$ are considered to have higher significance than tests resulting in $p<0.05$ (2008, p. 123). Each operational definition of the key characteristics is tied to the testable research hypotheses, so that results obtained from the rating scales determine the significance of these hypotheses. Therefore when non-parametric tests are performed to obtain the percentages of favourable responses or mean ranks, the $p$ values determine the significance of the difference between these responses or ranks along with the acceptance of the research hypotheses. For example, the mean ranks of a hypothesis determining the ease of using rotary, pushbutton, and slide interfaces were 2.58, 1.73 and 1.68 respectively, at $p<0.05$. This result implies that the rotary interface is easier to use than the pushbutton and sliding interfaces, and the differences between their mean ranks is significant as $p<0.05$.

The Statistical Package for Social Sciences [SPSS] Version 15.0 for Windows was used to perform non-parametric tests to calculate the percentages of favourable responses, the mean ranks, and p-values. The Cochran's $Q$ test was performed for calculating the statistical significance of tests involving binary response variables, while the Friedman's test was used for tests involving more than two response variables.

The qualitative data is coded using grounded theory coding, which is a form of content analysis that can be used to conceptualise the core issues 
from the gathered data. Allan argues that the data analysis in grounded theory involves searching out the concepts behind the actualities by looking for codes, then concepts and finally categories (2003, p. 1). The two goals of grounded theory include formulating hypotheses based on conceptual ideas, and discovering participants' main concerns and how they continually try to resolve it. Glaser and Strauss have claimed that while using grounded theory, "no preconceived ideas" should be forced on the data by looking for evidence to support established ideas (1967). However, Allan identified two difficulties with this approach: firstly, qualitative research requires a definite agenda as time and resource constraints prohibit unfocussed investigation; secondly, although the conceptualisation of coding is described, no definite mechanism is prescribed for performing the coding $(2003$, p. 8). Both these difficulties were overcome in Allan's study by identifying "key points" in the data and concentrating the analysis on these (p. 8).

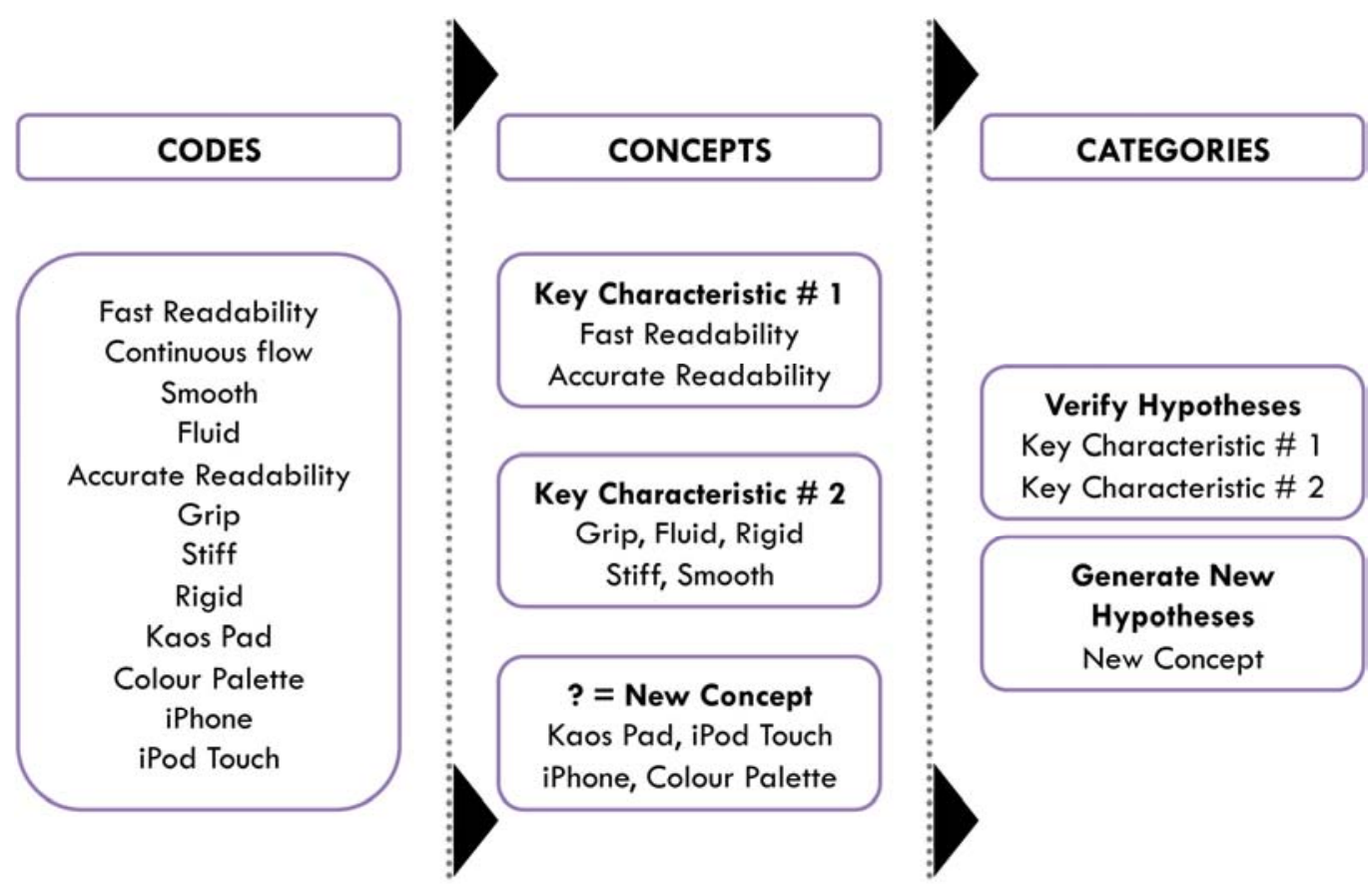

Figure 5 - An example of Grounded Theory coding 
The key characteristics are used as concentration points in the analysis of qualitative data gathered from the interactive studies. All design suggestions made by participants that are similar in nature to the key characteristics are coded under these characteristics to verify the testable hypotheses. For example, design suggestions relating to the content, format, accuracy and timeliness of information are coded under key characteristic \# 1. Any design suggestion that is different in nature from these characteristics is used to generate new design concepts about future tangible interfaces. The analysis serves the dual purpose of verifying the hypothetical predictions made at the start of the studies as well as generating a new set of hypothetical predictions for future testing.

\subsection{The Quality Control Measures: Validity checks}

Validity refers to the degree to which the empirical evidence and theoretical rationales of the studies accurately reflect or assess the specific concepts that this thesis research is attempting to measure. It is concerned with the studies' success at measuring the specific concepts of the thesis research. The principles of validity apply to interpretive and action inferences derived from quantitative test scores as well as inferences based on any other qualitative means of observing or documenting consistent behaviours or attributes (Messick, 1995, p. 741). Validation combines scientific inquiry with rational argument to justify or nullify the interpretations and use of any coding or summarisation of observed consistencies in an observation procedure. The following paragraphs examine the validity of the two questionnaire-based interactive studies in this thesis research. 


\subsubsection{Construct Validity}

Construct validity refers to whether the constructed scales measure or correlate with the theorised construct of the concepts. In scientific investigations, an unobservable idea of a one-dimensional concept must be 'constructed' into an easier-to-harder dimension or scale in the words of human language and graphics. For example, ease of use is a onedimensional concept that is constructed into an easier to harder scale: "Very easy to use - Easy to use - Difficult to use - Very difficult to use." Messick describes construct validity as the evidential basis for interpretation or meaning of the test results [that also includes contentand criterion-related evidence] where the measure used for testing is viewed as an extensible set of indicators of the construct (1995, p. 742). All one-dimensional concepts of this thesis research have been constructed into scales with easier-to-harder measurement levels. However, Messick has also identified two major threats to construct validity: construct underrepresentation and construct-irrelevant variance (1995, p. 742).

Construct underrepresentation is operative when the assessment is too narrow and fails to include important dimensions or facets of the construct. For example, the ease of use scale becomes too narrow if only two values are included, "Easy to use - Difficult to use." Constructirrelevant variance is operative when the assessment is too broad, containing extraneous dimensions that make performance of the task either too difficult or too easy for participants thereby adversely affecting the assessment of the construct. For example, the appearance scale of "Looks very much like - Looks like - Does not look like - Does not look like at all" has two extraneous variables namely "Looks very much like" and "Does not look like at all." Therefore to check whether the studies possess construct validity, the El study is performed in two stages: pilot and eventual. The pilot stage is to check whether participants are 
able to understand the different operational levels of the variables and perform the required control tasks in order to answer the questions. The instruments used in the eventual stage were modified using the results of the pilot stage.

\subsubsection{Internal Validity}

Internal validity refers to the care taken to conduct measurements in the study and decisions concerning what was and was not measured. Huitt describes internal validity as the rigor with which the study was conducted and the extent to which alternative explanations for any causal relationships have been taken into account (1998). The strategy of using experimental and non-experimental methods of gathering and evaluating data allows the ability to draw strong inferences about causal relationships between lighting control interfaces and end-user responses. Minke argues that although repeated measures design provides great benefits, there are certain other internal validity issues that must be addressed (1997). In a repeated measures design the aspect of presenting different levels of the independent variables in a particular sequence can lead to "carryover" effects from one treatment to another which may prove detrimental to the study (Lane).

One kind of carryover effect that can possibly cause a variance in the measurement of the dependent variables is called Practice effect. Kaufman describes practice effect as an improvement in performance of the task as a result of repeated practice with similar tasks during the course of multiple testing (2003). Additionally, presenting a sequence of interfaces to participants during multiple tests can lead to a Placebo effect. Carroll describes the placebo effect as a measurable or observable improvement in behaviour not attributable to an invasive treatment that has been administered (2003). Minke further argues that 
these threats to internal validity can be controlled through Counterbalancing (1997). Wendorf argues that as only the treatment administered first is immune to such effects, a common solution is to employ enough testing orders to ensure the equal occurrence of each experimental treatment at each stage in the test (1997). Cozby further argues that all possible sequences of presentation of the various levels of the independent variable should be included in the test in order to completely counterbalance the sequence of conditions (2001, p. 138). Therefore all possible sequences of lighting control interfaces were introduced to participants in the interactive studies.

\subsubsection{External Validity}

External validity refers to the validity of generalised causal inferences in scientific studies. Brewer argues that inferences about the cause-effect relationships between the dependent and independent variables possess external validity if they can be generalised from their unique and idiosyncratic settings, procedures and participants to other populations and conditions (2000, pp. 10-12). Lincoln and Guba further argue that within the qualitative research paradigm, external validity is replaced by the concept of transferability, which is the ability of research results to transfer to situations with similar parameters, populations and characteristics (1986). Therefore, studies possess high degrees of external validity when its inferences can be reasonably applied to the sample that was drawn from the target population and to the universe of other populations across time and space. Minke argues that in repeated measures design, even with counterbalancing carryover effects can raise issues involving external validity (1997).

The most common threat to external validity comes from the fact that experiments using human participants often employ small samples 
obtained from a single geographic and cultural location with idiosyncratic features. This is because definite conclusions cannot be drawn about whether the cause-effect relationships actually apply to people in other geographic locations or without these features. Wojciechowski and Zakrzewska argue that interface designers should take into consideration user needs and anticipations that arise from cultural preferences as people from different cultures [countries] have different expectations, metaphors and patterns of behaviour (2006, p. 465). To check whether the studies possess external validity, the pilot and eventual stages of the El study were conducted in two different geographical and cultural locations with different kinds of populations. India and New Zealand being my home and host countries respectively provided the two different geographical and cultural locations required for conducting the two stages of the study. The pilot stage was conducted at Anna University - Chennai, India and the eventual stage at Victoria University of Wellington, New Zealand. The results of these two stages were then compared for statistical interaction.

\subsection{Chapter Inference}

Chapter 5 has described the designs of the interactive studies, which focus on involving end-users in reactionary and participatory roles. These studies are part of the process of observing and documenting end-user behaviour with lighting control interfaces. Strategies of survey research involving experimental and non-experimental methods were used to study this causal relationship between end-users and lighting control interfaces. The process of evaluating usability and end-user experience of lighting control interfaces is done in two parts: an Existing interface [EI] study and a Tangible interface [TI] study. The El study is designed to 
obtain end-user opinions and desirable designs of better existing and future tangible interfaces. Responses obtained from El study were used to design prototypes of tangible interfaces, which were then tested and evaluated in the TI study. Target populations were sampled for both these studies. Studying causal relationships involves manipulating the independent variables or lighting control interfaces, and measuring the dependent variables or end-user responses in terms of the key characteristics.

Empirical evaluation requires operational definitions of the types of interfaces used and user-dependent key characteristics. The three types of physical interfaces used for manually controlling luminous intensity and colour were pushbutton, rotary and slide interfaces. Screen-based virtual interfaces were used for programming and recalling preset scenes. The two key characteristics evolved in Chapter 4 are given operational definitions. Richness of representation is a key characteristic that is given three operational definitions in terms of appearance, accuracy, and speed of learning the control information. Richness of reciprocation is a key characteristic that is given two operational definitions in terms of grabbability and responsiveness of interface objects. The evaluation tool merges ease of use with the operational definitions of the two key characteristics:

Appearance - The physical designs and/or external representations of interfaces should enable easier recognition of their intended control functions and operations.

Accuracy - The physical designs and/or external representations of interfaces should enable accurate selection of the desired lighting scenarios. 
Learning Speed - The physical designs and/or external representations of interfaces should enable quicker recognition of their intended control functions and operations.

Grabbability - The movable parts of interfaces should be easy to grab, and feel.

Responsiveness - The physical designs and external representations of interfaces should provide noticeable visual responses.

Ease of Use - The interfaces should be user-friendly.

A questionnaire was developed to obtain quantitative data through experimental methods and qualitative data through non-experimental methods. Testable hypotheses were formulated at the beginning of each interactive study. The quantitative data was analysed using inferential statistics to test these hypotheses. The qualitative data was analysed using grounded theory coding to either verify these hypotheses or generate new hypotheses. The El study was conducted in two stages to counter construct validity threats: pilot and eventual. Quantitative and qualitative data gathering, and counterbalancing techniques were used to counter internal validity threats. The pilot and eventual stages of the El study were conducted in two different geographical locations with two different populations to counter external validity threats: India and New Zealand.

In Chapter 5, two interactive studies namely El and $\mathrm{TI}$ are designed to transform the key characteristics into tools for evaluating usability and end-user experience of lighting control interfaces. These studies scientifically observe and document the causal relationship between endusers and lighting control interfaces. The study designs will have to be implemented in order to obtain tangible results to support the arguments 
put forward by this thesis research. Chapters 6 and 7 describe the implemented procedure and results of the El and TI studies respectively. 


\section{CHAPTER SIX}

\section{Evaluating Existing Interfaces}

\subsection{Chapter Intent}

Chapter 6 describes the portion of the interactive studies involving enduser evaluation of existing interfaces for lighting control. In Chapter 5, the design of two interactive studies to test the use as evaluation tools of the key characteristics from Chapter 4 is described: Existing interface [EI] and Tangible interface [TI] study. The El study has to be activated to enable end-user evaluation of the usability and end-user experience of existing interfaces. This involves formulating testable hypotheses, subdividing the study into pilot and eventual stages, and selecting participants for these stages. The execution of the study requires fabrication of different types of existing interfaces that will be used as independent variables. Once the interfaces are fabricated, the pilot stage has to be executed to determine whether the participants are able to understand the overall testing procedure. The results of the pilot stage will also determine whether the instruments used in the study require alteration. Once the study instruments are altered, the eventual stage has to be executed to achieve a second set of results. The results obtained from the pilot and eventual stages have to be analysed and compared for testing hypotheses, or combined for generating hypotheses. These results will also determine the validity of the study. The outlook is towards evaluating and improving designs of existing interfaces as well as designing future tangible interfaces. 
Section 6.2 formulates testable hypotheses, subdivides the El study into pilot and eventual stages, and selects participants for these stages. Section 6.3 describes the methods of fabricating physical and virtual interfaces. Section 6.4 describes the execution and results of the pilot stage. Section 6.5 describes the alterations done to the instruments used in the study based on the results of the pilot stage. Section 6.6 describes the execution and results of the eventual stage. Section 6.7 compiles and analyses the results of both the stages in order to validate the El study. Section 6.8 then summarises Chapter 6, and draws inferences for executing the Tl study in Chapter 7.

\subsection{Activation: El study}

The primary step for activating the El study is to formulate testable hypotheses from the first five hypothetical requirements for end-user interface with personalised lighting controls described in Chapter 3. The study is executed in two stages for ensuring quality control: pilot and eventual. Participants have to be selected for the execution of both these stages.

\subsubsection{Formulation - Hypotheses}

In the following paragraphs, the first five hypotheses describing end-user requirements for recognition and exploration are turned into statements that can be tested and verified in quantitative and qualitative terms. The differences between the percentages of favourable responses or mean ranks, and the p-value of tests will determine the significance of these hypotheses. 


\subsubsection{Hypothesis \# 1}

End-users learn to use a lighting control interface quickly and effectively when its physical design richly represents its intended control function and operational mechanics.

- Physical designs of rotary and slide interfaces enable quicker recognition and more effective operation of the luminous intensity control function than pushbutton interfaces.

- Physical designs of pushbutton interfaces enable quicker recognition and more effective operation of the luminous colour control function than rotary and slide interfaces.

\subsubsection{Hypothesis \# 2}

End-users learn to use a lighting control interface quickly and effectively when the iconography and typography depicted on the interface richly represents luminous colours or intensities, and lighting layers or scenes.

- Expressive iconographic and typographic representations of luminous intensities/colours enable quicker recognition and more accurate selection of the desired luminous intensities/colours than no representations.

- Expressive iconographic and typographic representations of lighting layers/scenes enable quicker recognition and more accurate selection of the desired lighting layers/scenes than representations with individual alphanumeric characters.

\subsubsection{Hypothesis \# 3}

End-users experience the graspable and material properties of an interface when its control handles provide rich haptic responses that appeal to the sense of touch. 
- Interface control handles with larger surface areas are more acceptable than control handles with small surface areas.

- Interface control handles with textured gripping surfaces are more acceptable than control handles without these surfaces.

\subsubsection{Hypothesis \# 4}

End-users prefer an interface with control handles that offer easy manipulation by an effortless shift in the gripping position.

- Interface control handles that provide an effortless shift in the gripping position are more acceptable than interfaces that do not provide an effortless shift.

\subsubsection{Hypothesis \# 5}

End-users experience the conversational property of an interface when it provides rich visual responses by a synchronic visual link to the changes in spatial illumination.

- Interfaces that provide noticeable visual changes during manipulation are considered more responsive than interfaces that do not provide these changes.

\subsubsection{Subdivision - Pilot and Eventual stages}

The subdivision of the El study into two stages is done mainly to test its construct and external validity. The pilot stage of the study is a "trial run" of the eventual stage that will reveal whether participants understand the instructions, whether the total experimental setting is plausible, and whether any confusing questions are being asked (Cozby, 2001, p. 158). The analysis of the quantitative and qualitative data gathered from both these stages is categorised to test the five 
hypotheses and generate new hypotheses for future testing in Section 6.7 .

\subsubsection{Selection - Participants}

The two stages of the study required two different populations from two different geographical and cultural locations to counter external validity threats. The participants selected for the pilot stage should ideally be similar to the target sample selected for the eventual stage in order to arrive at definite results (Giles, 2002, p. 113). However, care had to be taken that no participant of the target population sampled for the eventual stage was involved in the pilot stage of the study to avoid repetition. The School of Architecture and Planning, Anna University Chennai in India was the target location for sampling population for the pilot stage. The Schools of Architecture and Design, Victoria University of Wellington in New Zealand was the target location for sampling population for the eventual stage. A list of all the students and staff members from both the schools was made and thirty participants were approached randomly from each of these lists and asked to participate in the study. Therefore any form of repetition was avoided as a completely different and 'fresh' set of participants was selected for each stage.

The fact that participants were chosen from two entirely different socioeconomic, geographical and cultural backgrounds was accounted for in the questions relating to previous experience with lighting controls at the beginning of the El study questionnaire in Appendix 1. Participants in India showed a high level of interest for participation in the study all thirty [100\%] participants who were first approached agreed to participate. This high interest level could be accounted to the fact that this was the first time a research study based on lighting was being 
conducted at their university. However, the interest level of participants in New Zealand was slightly less as five of the first thirty [ $>15 \%$ ] participants approached declined citing reasons such as unavailability or lack of interest. Therefore five additional participants were approached at the eventual stage to complete the sample size of thirty. As it was assumed that most participants from India would have lesser previous experience with lighting controls in comparison to participants from $\mathrm{New}$ Zealand due to their respective socio-economic backgrounds, a brief introduction about lighting controls was provided at the start of the study for participants from both stages.

\subsection{Fabrication: Interfaces}

The El study required fabrication of physical and virtual interfaces for performing three different kinds of lighting control functions: luminous intensities, luminous colours and preset scenes. Physical interfaces comprising pushbutton, rotary, and slide interfaces are used for controlling luminous intensity and colours. Virtual interfaces comprising different kinds of iconographic and typographic representations are used for recalling preset scenes.

\subsubsection{Manual Control Interfaces - Luminous intensity}

The three different types of physical interfaces for manually controlling luminous intensity were selected based on their ease of availability, both in the Indian and New Zealand markets. The Rania model from the manufacturer Lutron with a dual increase and decrease function was used as the pushbutton interface. The digital interface consists of three pushbuttons, one for turning the lights on/off, and one each for increasing and decreasing the luminous intensity. The two buttons used for 
increasing and decreasing luminous intensity are labelled with an up and down arrow respectively. The interface memorises the last luminous intensity level and includes a green luminous LED indicator to indicate the selected intensity level.

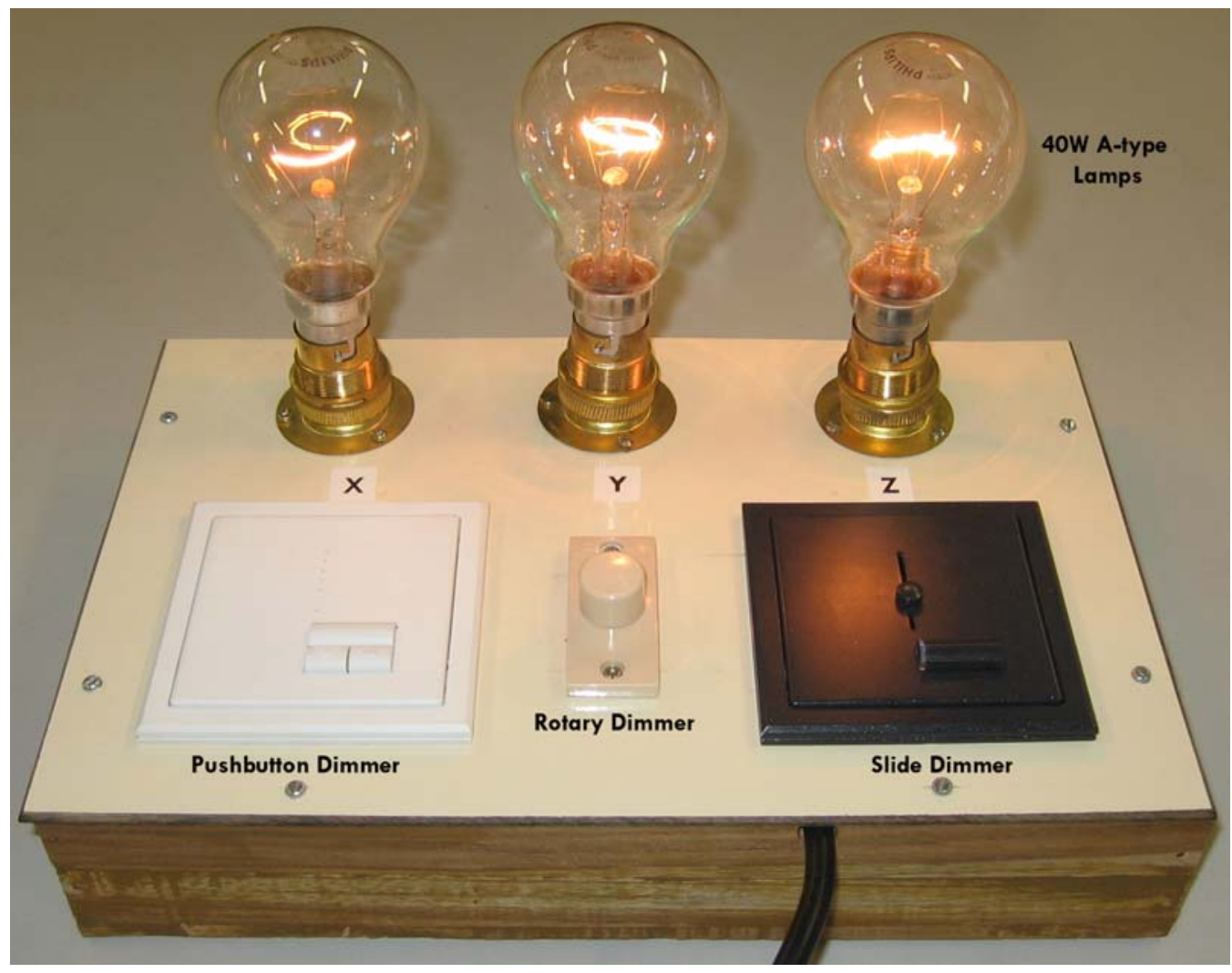

Figure 6 - Manual control interfaces for luminous intensity

The rotary-switch model from the manufacturer Anchor was used as the rotary interface. The dimmer has an in-built switch to turn on/off lights along with the function of increasing and decreasing luminous intensity by rotation of the dial. A red LED indicator near the rotary dial indicates whether the light has been turned on/off. However, this model does not have any representations to indicate the selected luminous intensity level.

The Lyneo model from Lutron with an adjustable round slider to perform the increase and decrease function was used as the slide interface. The 
model consists of a contoured pushbutton on the side of the slider to turn the light on/off. The position of slider indicates the selected luminous intensity level.

The three lamps used for the experiment were 40W A-type clear incandescent lamps from Philips, and each dimming interface was used to control the luminous intensity of one lamp. All the three dimming interfaces and their respective lamps were installed on a wooden box for participants to directly view the changes in luminous intensity of the lamps while using these interfaces. 'Experiment 1' on the El study questionnaire in Appendix 1 first asked participants to visually study these three interfaces before rating their appearance as interfaces for dimming control on the 'Appearance' scale. It then asked participants to grab, feel and move its movable parts before rating the ease with which they are able to grab these parts on the 'Grabbability' scale. It finally asked participants to provide reasons for their rating choices.

Although each of these interfaces provides the mentioned additional functions, participants were not informed about any of these functions that can be performed by the interfaces besides controlling luminous intensity. Any form of exploration of the additional functions was left entirely up to the participants. 'Experiment 2' on the El study questionnaire in Appendix 1 asked participants to first use these interfaces individually to change the luminous intensity of each of these lamps before rating the ease with which they are able to use these interfaces on the 'Ease of Use' scale. It then asked participants to use these interfaces individually to first select a luminous intensity level [50\%] of the lamp, then select the minimum luminous intensity level, and again try to select the previous luminous intensity level [50\%] of the lamp before rating the accuracy with which they are able to repeat the 
selection on the 'Accuracy' scale. It deliberately asked participants to select a luminous intensity level of $50 \%$ as the green luminous LED display of the pushbutton interface and slider position of the slide interface provide an appropriate indication of this level. It also asked participants to use these interfaces individually to 'gradually' increase or decrease the luminous intensity level of these lamps, and observe the gradual change in luminous intensity level of these lamps as they operate these interfaces before rating the responsiveness of these interfaces on the 'Responsiveness' scale. Although the pushbutton provides a step-wise control and the rotary and slide interfaces provide a continuous control, the word 'gradual' was deliberately used for participants to identify the difference between step-wise and continuous control. It finally asked participants to provide any additional comments on the use of these interfaces.

\subsubsection{Manual Control Interfaces - Luminous colour}

The three different types of physical interfaces for manually controlling luminous colours were custom fabricated for this study. A tri-colour RGB LED strip of $30 \mathrm{~cm}$ length was used as the primary light source. The property of additive colour mixing from the three primary colours of the LED enabled a colour output of any choice. However, the number of colour combinations for this study was limited to seven: red, yellow [red + green], green, cyan [green + blue], blue, magenta [blue + red] and white $[$ red + green + blue]. A micro-controller was custom programmed to enable the additive colour mixing of the seven colours from the three primary colours with pulse-width modulation.

An eight-button panel was used as a pushbutton interface, where participants can use each button on the panel to select a corresponding colour with one button used for turning off the LED strip. A rotating 
potentiometer was used as a rotary interface, and a sliding potentiometer was used as a slide interface where participants can continuously rotate or slide the interface to select the seven colours starting from the 'Off' position. Therefore the colour chosen on the LED strip depends on the corresponding position of the rotary dial or slider. These interfaces were installed on a control box that houses the microcontroller. 'Experiment 1' on the El study questionnaire in Appendix 1 asked participants to visually study these three colour control interfaces as well before rating their appearance as interfaces for luminous colour control on the 'Appearance' scale. It finally asked participants to provide reasons for their rating choices.

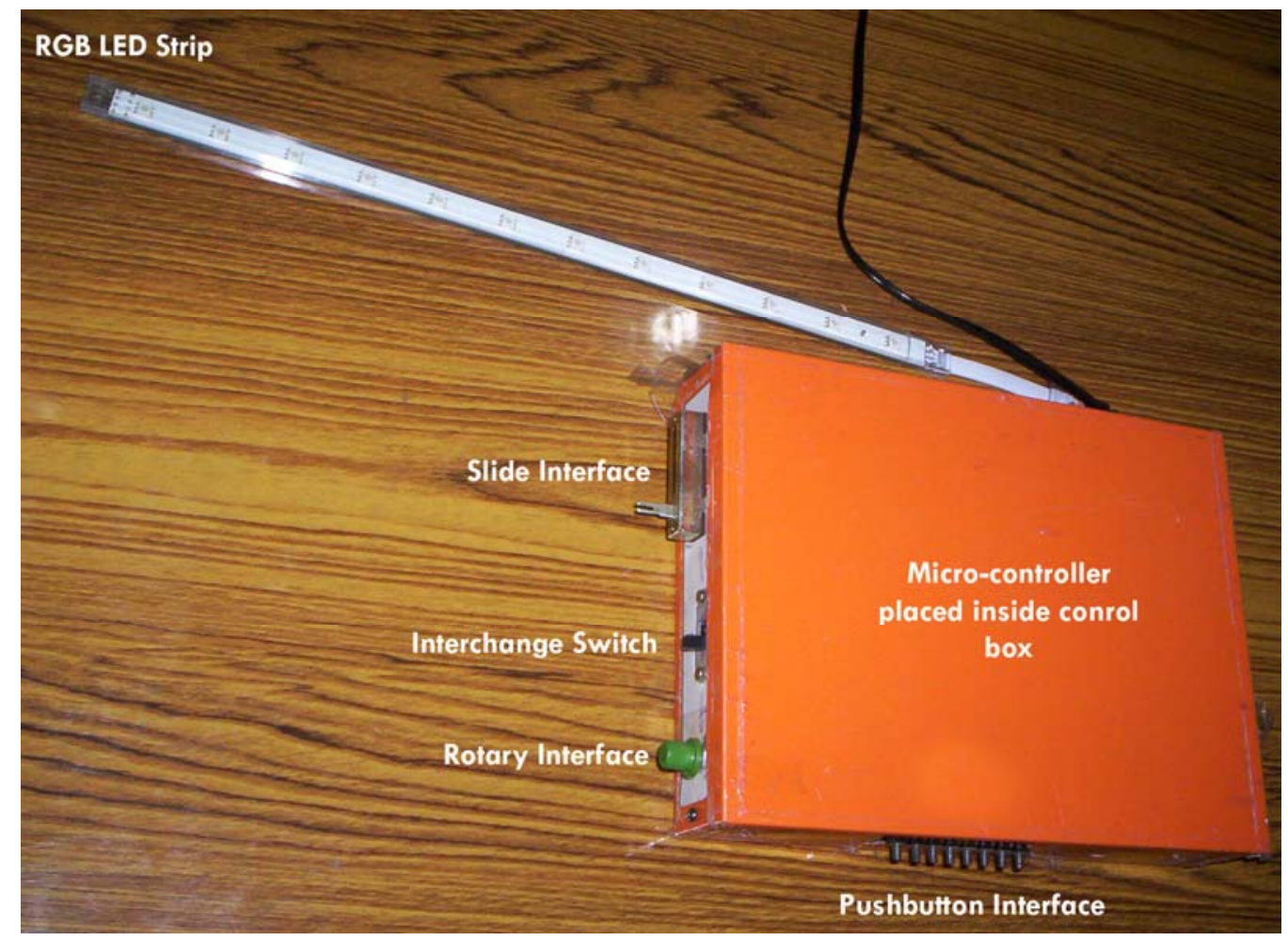

Figure 7 - Manual control interfaces for luminous colour [El study: Pilot stage]

An interchange switch was also installed on the box to enable switching between the three types of interfaces. Therefore unlike the dimming interfaces, the three interfaces are used to select different luminous 
colours from a single LED strip and participants can switch between the three interfaces using the interchange switch. 'Experiment 3' on the El study questionnaire in Appendix 1 first asked participants to use these interfaces individually to change the luminous colours while directly viewing the LED strip before rating the ease with which they are able to use these interfaces on the 'Ease of Use' scale. It then asked participants to use these interfaces individually to first select a luminous colour on the LED strip, then select a different luminous colour, and again try to select the previous luminous colour of the LED strip before rating the accuracy with which they are able to repeat the selection on the 'Accuracy' scale. It also asked participants to use these interfaces individually to 'gradually' change the luminous colours, and observe the gradual change in colours of the LED strip as they operate these interfaces before rating the responsiveness of these interfaces on the 'Responsiveness' scale. Although the pushbutton provides a step-wise control and the rotary and slide interfaces provide a continuous control, the word 'gradual' was deliberately used for participants to identify the difference between step-wise and continuous control. It finally asked participants to provide any additional comments on the use of these interfaces.

\subsubsection{Preset Control Interfaces - Recall scenes}

Five different designs of virtual interfaces were custom fabricated for recalling four preset scenes in a conference room. Images of a conference room depicting four different lighting scenes were downloaded from the Lutron website. The 'General Meeting' scene is for focussing lighting on a conference table; 'Maintenance' scene is for switching on all the lights in the room for maintenance and cleaning; ' $A / V$ Presentation' scene is for selecting a low-level lighting during audio-visual presentations; and 'Night Light' scene is for maintaining a low-level general lighting when the room is unoccupied. The five different designs 
represent these scenes in the form of alphanumeric characters, labelled scenes, purely iconographic images of the scene, purely typographic phrases describing the scene, and a combination of iconographic images and typographic phrases.

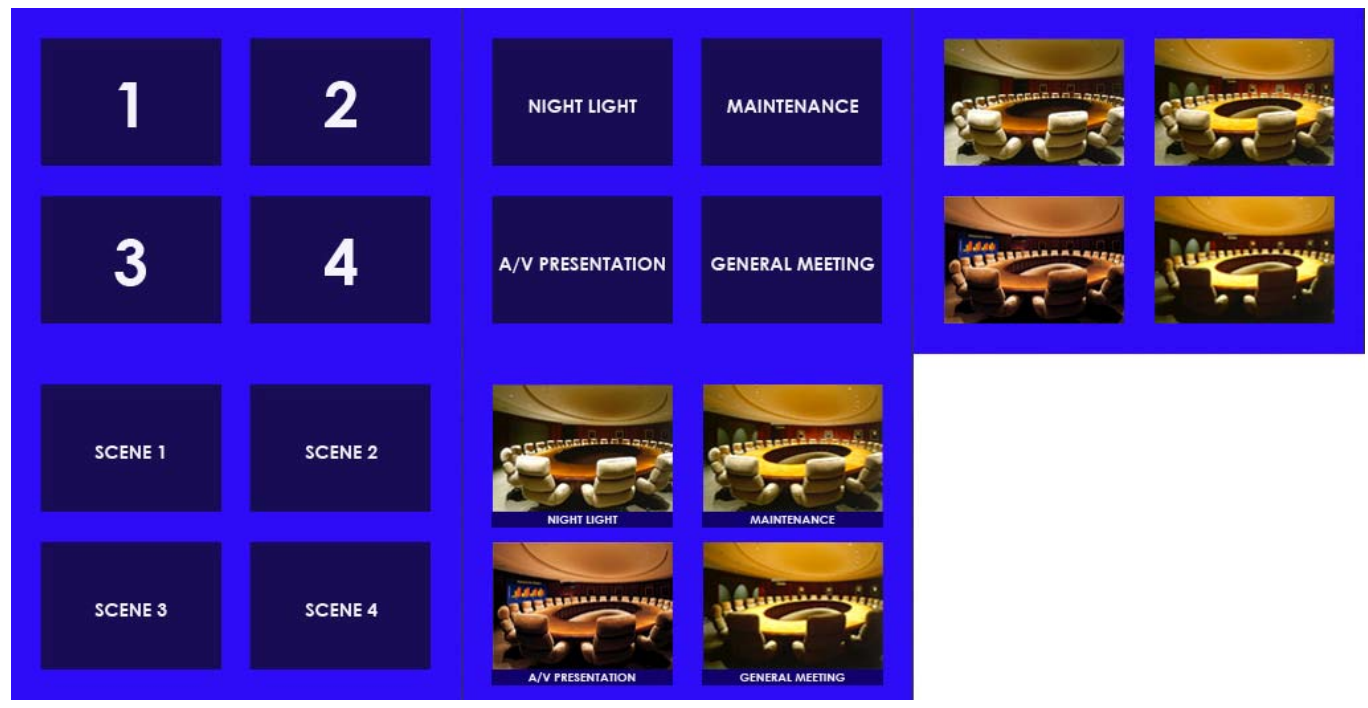

Figure 8 - The five preset control interfaces

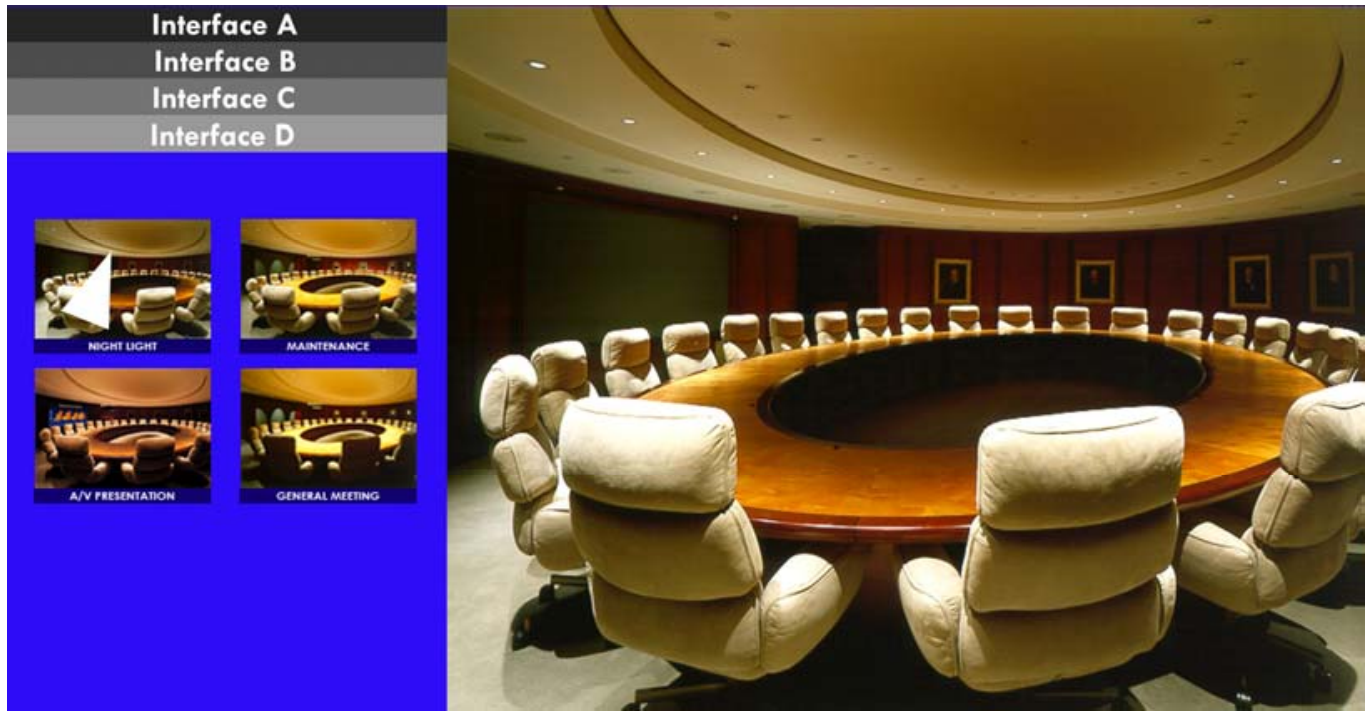

Figure 9 - A split-screen view of the web page with an interface and cursor position selecting the 'Night Light' scene

The experimental set-up with the virtual interfaces consists of a web page with a split-screen view that has the five different interface types 
on the left and a projection of four different preset scenes on the right. The five different types of interfaces work on a 'drop-down' menu and every time participants click on one of the interface menu, the display 'drops-down' to reveal the entire interface. Participants can further click on each of the four preset scene options on the interface to view the projected lighting scene on the right screen.

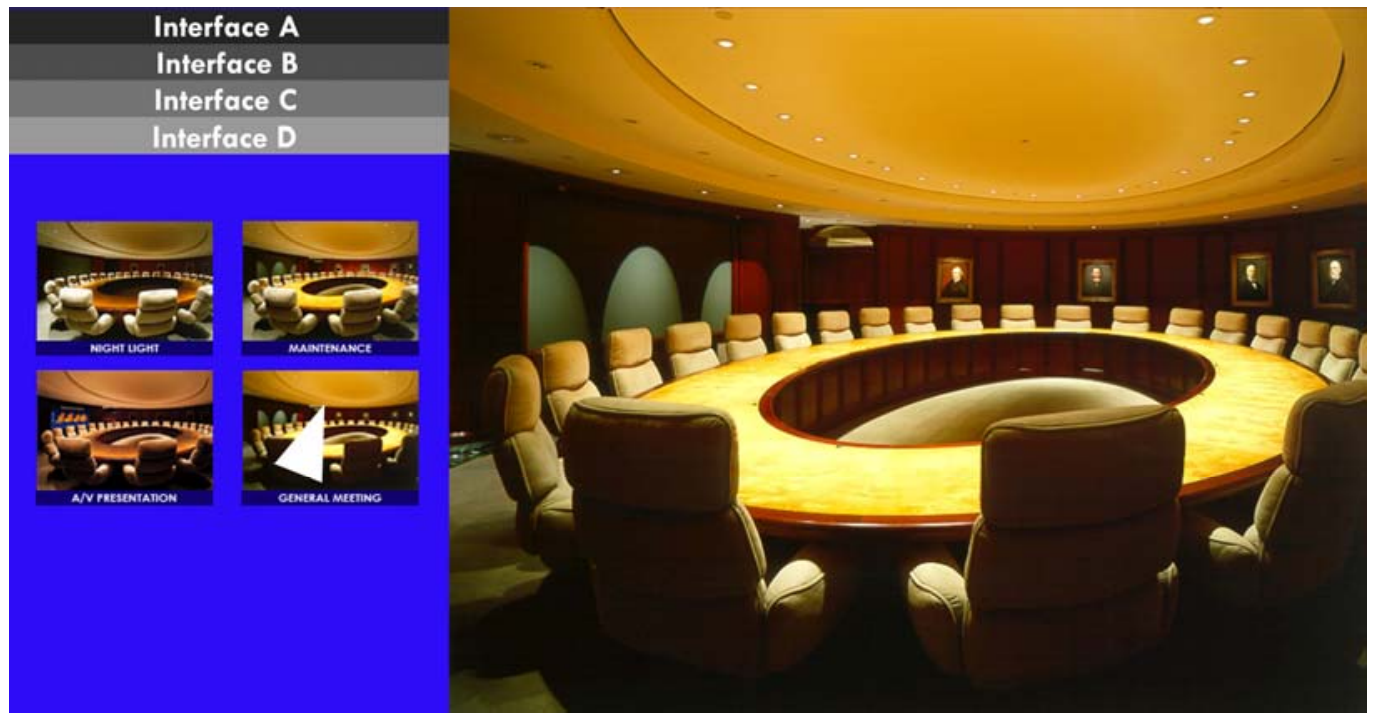

Figure 10 - A split-screen view of the web page with an interface and cursor position selecting the

‘General Meeting' scene

The scene selection web page was set on a Macintosh notebook screen and participants used pointing devices such as a mouse or touchpad to select the different scenes. 'Experiment 4' on the El study questionnaire in Appendix 1 first asked participants to use each of the five scene selection interfaces to select the different lighting scenes before rating the accuracy with which these interface were able to describe the different scenes on the 'Accuracy' scale. It then asked participants to rate the time taken to understand the different lighting scenes from these interfaces on the 'Learning Speed' scale. It finally asked participants to provide any additional comments on the use of these interfaces. 


\subsection{Execution: Pilot stage}

The pilot stage was executed within the premises of the School of Architecture and Planning, Anna University - Chennai in India. A brief demographic analysis of the thirty participants showed that twenty-four are from the age group of 16-24 years, one from the age group of 2534 years, two from the age group of $35-44$ years, two from the age group of 45-54 years, and one from the age group of 55 and above. Eight of the participants are male while twenty-two are female. All thirty participants have used lighting control interfaces before this study for turning on/off lights while seventeen have used interfaces for controlling luminous intensity. This shows that the socio-economic and cultural background does affect participants' previous experience with using interactive lighting control, as almost half population have not used them prior to this experiment. Six participants have used interfaces for controlling luminous colour while four participants have used interfaces for recalling preset scenes. As very few participants have used interfaces for luminous colour and preset control, it was assumed that the overall assessment of these interfaces would tend to be fair and unbiased, as most of them have used these interfaces for the first time. The following paragraphs describe the results of the pilot stage.

\subsubsection{Results - Evaluating interfaces}

The test scores for each of the operational definitions obtained from the quantitative data using inferential statistics are listed in Tables 3-6. Any other qualitative comments made by participants were coded, conceptualised and categorised using the grounded theory coding described in Section 5.5.3 under each of the operational definitions. 


\subsubsection{Evaluating Interfaces - Appearance}

Participants rated the appearance of the slide interface over the rotary and pushbutton interfaces as an interface for controlling luminous intensity. The percentage of favourable responses for the slide interface was $86.7 \%$, the rotary interface was $56.7 \%$, and the pushbutton interface was $50.0 \%$. These differences in percentages are significant as $p<0.05$. Additionally, three out of the thirty [10\%] participants commented that although the rotating action of the rotary interface is recognisable, they are more familiar with its usage as a "regulator" for controlling "fan speed" rather than luminous intensity.

Participants found no difference in the appearance of the pushbutton, rotary, and slide interfaces for controlling luminous colours. As $p>0.05$, it is accepted that the differences in percentages of responses were due to random error.

\subsubsection{Evaluating Interfaces - Accuracy}

Participants rated the pushbutton interface as more accurate than the sliding and rotary interfaces for selecting their desired luminous intensity level. The mean rank for the pushbutton interface was 2.27, the slide interface was 2.03 , and the rotary interface was 1.70 . These differences in mean ranks are significant as $p<0.05$.

Participants rated the pushbutton interfaces as more accurate than the slide and rotary interfaces for selecting their desired luminous colours. The mean rank for the pushbutton interface was 2.70, the sliding interface was 1.67 , and the rotary interface was 1.63 . These differences in mean ranks are highly significant as $p<0.001$. 
Participants rated the interface with a combination of iconic and textual representations of the depicted scenes as the most accurate, and the interface with numerical representations as the least accurate for recalling preset lighting scenes. The mean ranks for the five different interfaces were 4.67 for the combined iconic and typographic, 3.63 for purely iconic, 3.33 for purely typographic, 1.85 for labelled scenes, and 1.52 for numerical representations of the scenes. These differences in mean ranks are highly significant as $p<0.001$.

\subsubsection{Evaluating Interfaces - Learning speed}

Participants took the least amount of time to learn about the preset lighting scenes for recall from the interface with a combination of iconic and typographic representations of the depicted scenes, and most amount of time with the interface with only numerical representations. The mean ranks for the five different interfaces were 4.53 for the combined iconic and typographic, 3.90 for purely iconic, 3.12 for purely typographic, 1.92 for labelled scenes, and 1.53 for numerical representations of the scenes. These differences in mean ranks are highly significant as $p<0.001$.

\subsubsection{Evaluating Interfaces - Grabbability}

Participants rated the rotary interface as easier to grab than the sliding and pushbutton interfaces while controlling luminous intensity. The percentage of favourable responses for the rotary interface was $86.7 \%$, the pushbutton interface was $53.3 \%$, and the slide interface was $43.3 \%$. These differences in percentages are significant as $p<0.05$. 


\subsubsection{Evaluating Interfaces - Responsiveness}

Participants found no difference in the responsiveness of the pushbutton, rotary, and slide interfaces for controlling luminous intensity. As $p>$ 0.05 , it is accepted that the differences in mean ranks were due to random error.

Participants found no difference in the responsiveness of the pushbutton, rotary, and slide interfaces for controlling luminous colours. As $p>0.05$, it is accepted that the differences in mean ranks were due to random error.

\subsubsection{Evaluating Interfaces - Ease of use}

Participants rated the rotary interface as easier to use than the slide and pushbutton interfaces for controlling luminous intensity. The mean rank for the rotary interface was 2.58 , the pushbutton interface was 1.73 , and the slide interface was 1.68. These differences in mean ranks are highly significant as $p<0.001$.

Participants rated the slide and rotary interfaces as easier to use than the pushbutton interface for controlling luminous colours. The mean rank for the slide interface was 2.23, rotary interface was 2.18, and the pushbutton interface was 1.58. These differences in mean ranks are significant as $\mathrm{p}<0.05$.

Additionally, six out of thirty [20\%] participants commented that "rigid" or "stiff" interfaces that do not provide a "smooth" shift in gripping position are difficult to use and vice-versa. 


\subsubsection{Evaluating Interfaces - Summary}

End-user evaluation of existing interfaces shows that participants in India were able to identify the function of luminous intensity control easily from the appearance of slide interface in comparison to the rotary and pushbutton interfaces. They were able to select and repeat a selection of luminous intensity level accurately with the pushbutton interface, slightly less but accurately nevertheless with the slide interface, and the least amount of accuracy with the rotary interface. The larger dial of the rotary interface made it easier for the participants to grab in comparison to the smaller control handles of pushbutton and slide interfaces. Participants did not find any difference between the conversational interactive properties of these interfaces. However, they prefer using the rotary interface in comparison to the pushbutton or slide interfaces probably due the stiffness or rigidness of their control handles.

End-user evaluation of existing interfaces shows that participants in India were not able to identify the function of controlling luminous colour from the appearances of these interfaces. Similarly, they did not find any difference in the conversational interactive properties of these interfaces. They were able to select and repeat a selection of luminous colour much more accurately with the pushbutton interface in comparison to the slide and rotary interfaces. Participants found the slide interface easy to use, the rotary interface to be slightly less but easy nevertheless, and the pushbutton interface to be difficult to use probably due to the stiffness or rigidness of the buttons.

According to end-user evaluation of existing interfaces by participants in India, selection of desired lighting scenarios was quickest and most accurate with preset control interfaces that describe the preset scenes with a combination of text and images. Participants considered the 
interface with pure images to be more accurate and required less time in comparison to the interface with pure textual descriptions. Interfaces with labelled scenes and purely numerical representations were considered least accurate and required the highest amount of time for scene selection.

\begin{tabular}{|c|c|c|c|c|c|c|c|c|c|c|}
\hline \multirow[t]{2}{*}{ Type } & \multirow[t]{2}{*}{ Dimension } & \multicolumn{3}{|c|}{ Pushbutton } & \multicolumn{3}{|c|}{ Rotary } & \multicolumn{3}{|c|}{ Slide } \\
\hline & & $M$ & $S D$ & $\begin{array}{l}\text { Min- } \\
\text { Max }\end{array}$ & $M$ & $S D$ & $\begin{array}{l}\text { Min- } \\
\text { Max }\end{array}$ & $M$ & $S D$ & $\begin{array}{l}\text { Min- } \\
\text { Max }\end{array}$ \\
\hline \multirow{5}{*}{ 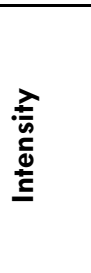 } & Appearance & 1.500 & 0.509 & $1-2$ & 1.567 & 0.504 & $1-2$ & 1.867 & 0.346 & $1-2$ \\
\hline & Accuracy & 2.467 & 0.730 & $1-3$ & 2.067 & 0.740 & $1-3$ & 2.267 & 0.692 & $1-3$ \\
\hline & Grabbability & 1.533 & 0.507 & $1-2$ & 1.867 & 0.346 & $1-2$ & 1.433 & 0.504 & $1-2$ \\
\hline & Responsiveness & 2.333 & 0.711 & $1-3$ & 2.633 & 0.615 & $1-3$ & 2.500 & 0.509 & $2-3$ \\
\hline & Ease of Use & 2.700 & 1.022 & $1-4$ & 3.667 & 0.547 & $2-4$ & 2.767 & 0.817 & $1-4$ \\
\hline \multirow{4}{*}{$\frac{\grave{3}}{0}$} & Appearance & 1.533 & 0.507 & $1-2$ & 1.367 & 0.490 & $1-2$ & 1.633 & 0.490 & $1-2$ \\
\hline & Accuracy & 2.900 & 0.305 & $2-3$ & 2.033 & 0.556 & $1-3$ & 2.067 & 0.640 & $1-3$ \\
\hline & Responsiveness & 2.367 & 0.615 & $1-3$ & 2.633 & 0.490 & $2-3$ & 2.533 & 0.571 & $1-3$ \\
\hline & Ease of Use & 2.567 & 0.817 & $1-4$ & 3.233 & 0.679 & $2-4$ & 3.267 & 0.583 & $2-4$ \\
\hline
\end{tabular}

Table 3 - [El study: Pilot stage] Summary of Means, Standard Deviations and Minimum-Maximum range for the different luminous intensity and colour control interfaces

\begin{tabular}{|c|c|c|c|c|c|c|}
\hline Type & Dimension & Pushbutton & Rotary & Slide & & \\
\hline & & \multicolumn{3}{|c|}{$\begin{array}{l}\text { (Does not look like) Looks like \% } \\
\text { Or (Hard to grab) Easy to grab \% }\end{array}$} & $p$ & $\begin{array}{l}\text { Cochran } \\
Q_{(d f=2)}\end{array}$ \\
\hline \multirow[t]{2}{*}{ Intensity } & Appearance & $(50.0) 50.0$ & (43.3) 56.7 & $(13.3) 86.7$ & 0.029 & 7.103 \\
\hline & Grabbability & $(46.7) 53.3$ & $(13.3) 86.7$ & $(56.7) 43.3$ & 0.005 & 10.692 \\
\hline \multirow[t]{2}{*}{ Colour } & Appearance & $(46.7) 53.3$ & (63.3) 36.7 & $(36.6) 63.3$ & 0.185 & 3.727 \\
\hline & & \multicolumn{3}{|c|}{ Mean ranks } & $p$ & $\chi^{2}(d f=2)$ \\
\hline \multirow[t]{3}{*}{ Intensity } & Accuracy & 2.27 & 1.70 & 2.03 & 0.048 & 6.083 \\
\hline & Responsiveness & 1.82 & 2.18 & 2.00 & 0.245 & 2.814 \\
\hline & Ease of Use & 1.73 & 2.58 & 1.68 & $<0.001$ & 17.712 \\
\hline \multirow[t]{3}{*}{ Colour } & Accuracy & 2.70 & 1.63 & 1.67 & $<0.001$ & 30.091 \\
\hline & Responsiveness & 1.83 & 2.15 & 2.02 & 0.334 & 2.193 \\
\hline & Ease of Use & 1.58 & 2.18 & 2.23 & 0.007 & 9.813 \\
\hline
\end{tabular}

Table 4 - [El study: Pilot stage] Percentages of responses and mean ranks for the different luminous intensity and colour control interfaces 


\begin{tabular}{|c|c|c|c|c|c|c|c|}
\hline Type & Dimension & & Numbers only & Text only & Icons only & Labels & Text + Icons \\
\hline \multirow{6}{*}{$\begin{array}{l}\bar{\Xi} \\
\Phi \\
\Phi \\
\text { ¿ }\end{array}$} & \multirow[t]{3}{*}{ Accuracy } & $M$ & 1.033 & 2.133 & 2.267 & 1.233 & 2.967 \\
\hline & & $S D$ & 0.183 & 0.629 & 0.521 & 0.504 & 0.183 \\
\hline & & Min-Max & $1-2$ & $1-3$ & $1-3$ & $1-3$ & $2-3$ \\
\hline & \multirow[t]{3}{*}{ Learning Speed } & $M$ & 1.133 & 2.100 & 2.567 & 1.337 & 2.967 \\
\hline & & $S D$ & 0.346 & 0.607 & 0.568 & 0.556 & 0.183 \\
\hline & & Min-Max & $1-2$ & $1-3$ & $1-3$ & $1-3$ & $2-3$ \\
\hline
\end{tabular}

Table 5 - [El study: Pilot stage] Summary of Means, Standard Deviations and Minimum-Maximum range for the different preset scene control interfaces

\begin{tabular}{|c|c|c|c|c|c|c|c|c|}
\hline Type & Dimensions & $\begin{array}{c}\text { Numbers } \\
\text { only }\end{array}$ & $\begin{array}{l}\text { Text } \\
\text { only }\end{array}$ & $\begin{array}{l}\text { Icons } \\
\text { only }\end{array}$ & Labels & $\begin{array}{l}\text { Text + } \\
\text { lcons }\end{array}$ & & \\
\hline \multirow{3}{*}{ Preset } & & \multicolumn{5}{|c|}{ Mean Ranks } & $p$ & $\chi^{2}(d f=4)$ \\
\hline & Accuracy & 1.52 & 3.33 & 3.63 & 1.85 & 4.67 & $<0.001$ & 93.790 \\
\hline & $\begin{array}{l}\text { Learning } \\
\text { Speed }\end{array}$ & 1.53 & 3.12 & 3.90 & 1.92 & 4.53 & $<0.001$ & 90.866 \\
\hline
\end{tabular}

Table 6 - [El study: Pilot stage] Mean ranks for the different preset scene control interfaces

\subsubsection{Results - Designing interfaces}

\begin{tabular}{|c|c|c|}
\hline & Participants' descriptions/drawings & No: of Participants \\
\hline \multirow{4}{*}{ 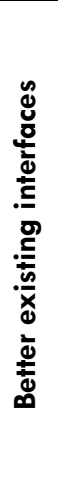 } & $\begin{array}{l}\text { One streamlined action for switching and dimming for } \\
\text { pushbutton and slide interfaces without additional On/Off } \\
\text { switch }\end{array}$ & 2 \\
\hline & Control handles with a surface "texture" or "grip" & 1 \\
\hline & $\begin{array}{l}\text { Iconic depictions in the form of a "graphical scale" or "colour } \\
\text { code" depicting the range of luminous intensity levels or } \\
\text { colours to improve the accuracy of interfaces }\end{array}$ & 4 \\
\hline & $\begin{array}{l}\text { Abstract graphics instead of actual images or text as a mode } \\
\text { of representing lighting scenes }\end{array}$ & 2 \\
\hline \multirow{4}{*}{ 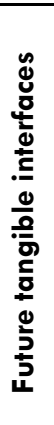 } & $\begin{array}{l}\text { One interface to perform all three functions of luminous } \\
\text { intensity, luminous colour, and preset scenes }\end{array}$ & 2 \\
\hline & $\begin{array}{l}\text { Handheld "touch-screen" based "remote" interfaces similar to } \\
\text { "iPod Touch" or "iPhone" with "screen representations" of } \\
\text { luminous intensity, luminous colour, and lighting scenes }\end{array}$ & 4 \\
\hline & $\begin{array}{l}\text { Interface that changes luminous intensity by sensing the } \\
\text { pressure provided by the hand }\end{array}$ & 1 \\
\hline & Interface that resembles everyday objects that people use & 1 \\
\hline
\end{tabular}

Table 7 - [El study: Pilot stage] Summary of participants' suggestions for most desirable interface designs 
1. For colour controlles, it may be a good

idea to have the colouts represented in the

Controlling device

For Eg.
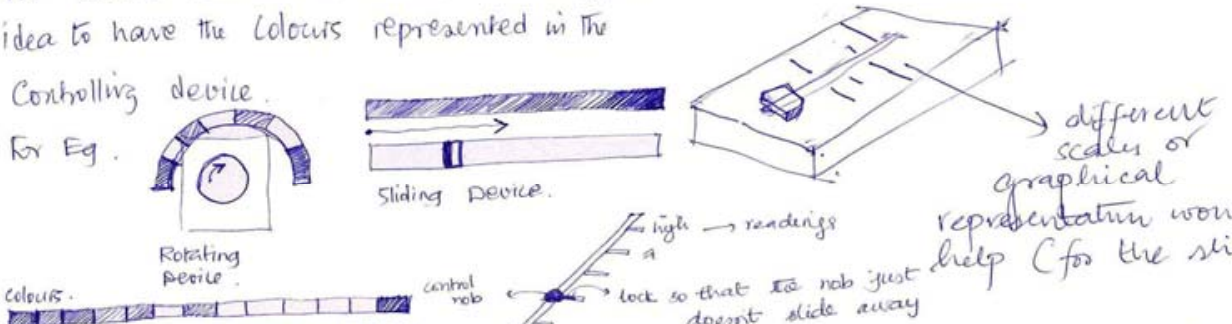

Sliding Device. araphical EIrey

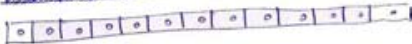

Pish Buttor option

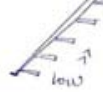

representatum would help (for the sliders)

2. For Dimmers too graphical illustrations might help.

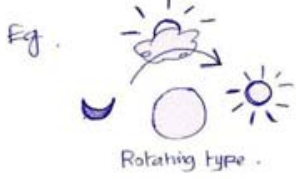

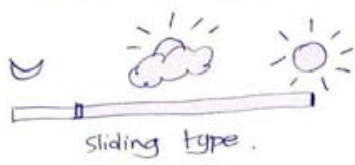

Scone sebets

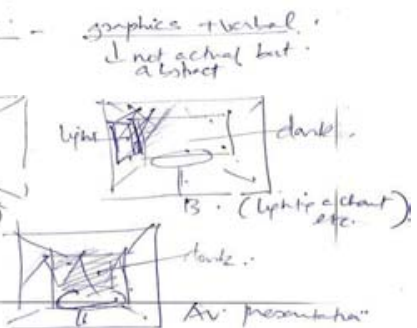

Figure 11 - Design suggestions for better existing interfaces [EI study: Pilot stage]

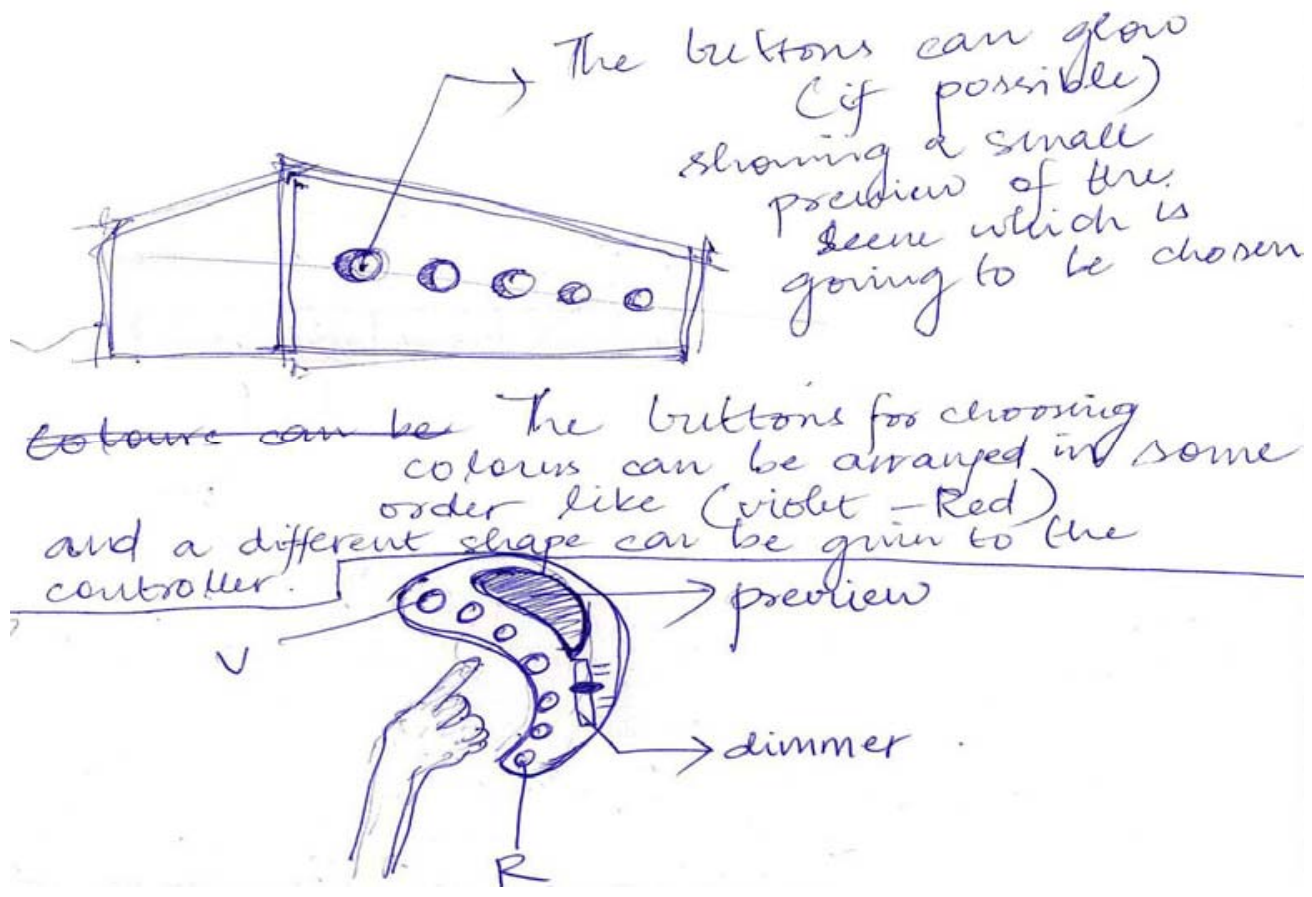

Figure 12 - Design suggestions for future tangible interfaces [El study: Pilot stage]

The qualitative data in the form of design suggestions made by participants are coded, conceptualised and categorised using the grounded theory coding described in Section 5.5.3 under two categories for designing interfaces: better existing and future tangible. The system 
used is to list all suggestions made by participants in terms of continuous prose, listed answers, or conceptual sketches. These are then quantified by grouping all similar descriptions under the number of participants who made these suggestions in Table 7.

\subsection{Alteration: Study instruments}

Flaws were identified with two instruments used in the study during the execution of the pilot stage. The first flaw was with the design of the numerical scale used in the close-ended questions of the questionnaire, and the second flaw was in the fabrication of the colour controller.

\subsubsection{Questionnaire}

The similar alphanumeric characters used in the scales of the close-ended questions and numerical representations of the four scenes on two of the scene recall interfaces led to participants misreading these values. Several participants required clarification while answering the questionnaire that the numerical characters used in the scale does not represent the four scenes. Therefore, an alteration was done in the questionnaire used in the eventual stage where the numbering style in the closed-ended questions using arithmetic numerals [1, 2, 3 and 4] was replaced with roman numerals [i, ii, iii and iv].

\subsubsection{Manual Control Interfaces - Luminous colour}

Two participants commented that the colour controller requires refinement. Additionally, the placement of the three interfaces on two different planes of the control box was observed to be a cause of inconvenience to the participants. The absence of any graspable control 
handle for the sliding interface and the small size of the pushbuttons caused further inconvenience. Therefore for the eventual stage, rotary and slide potentiometers with larger control handles, and an eight-button switch with larger buttons were used. The primary light source was also replaced with high-output RGB LEDs, which are encased inside an opalescent casing to enable seamless colour mixing. A new microcontroller was procured and programmed for use with the high-output LEDs. All the three interfaces were mounted on a single plane of a new control box. The interchange switch was mounted on a different plane of the control box to avoid misreading with the actual interfaces.

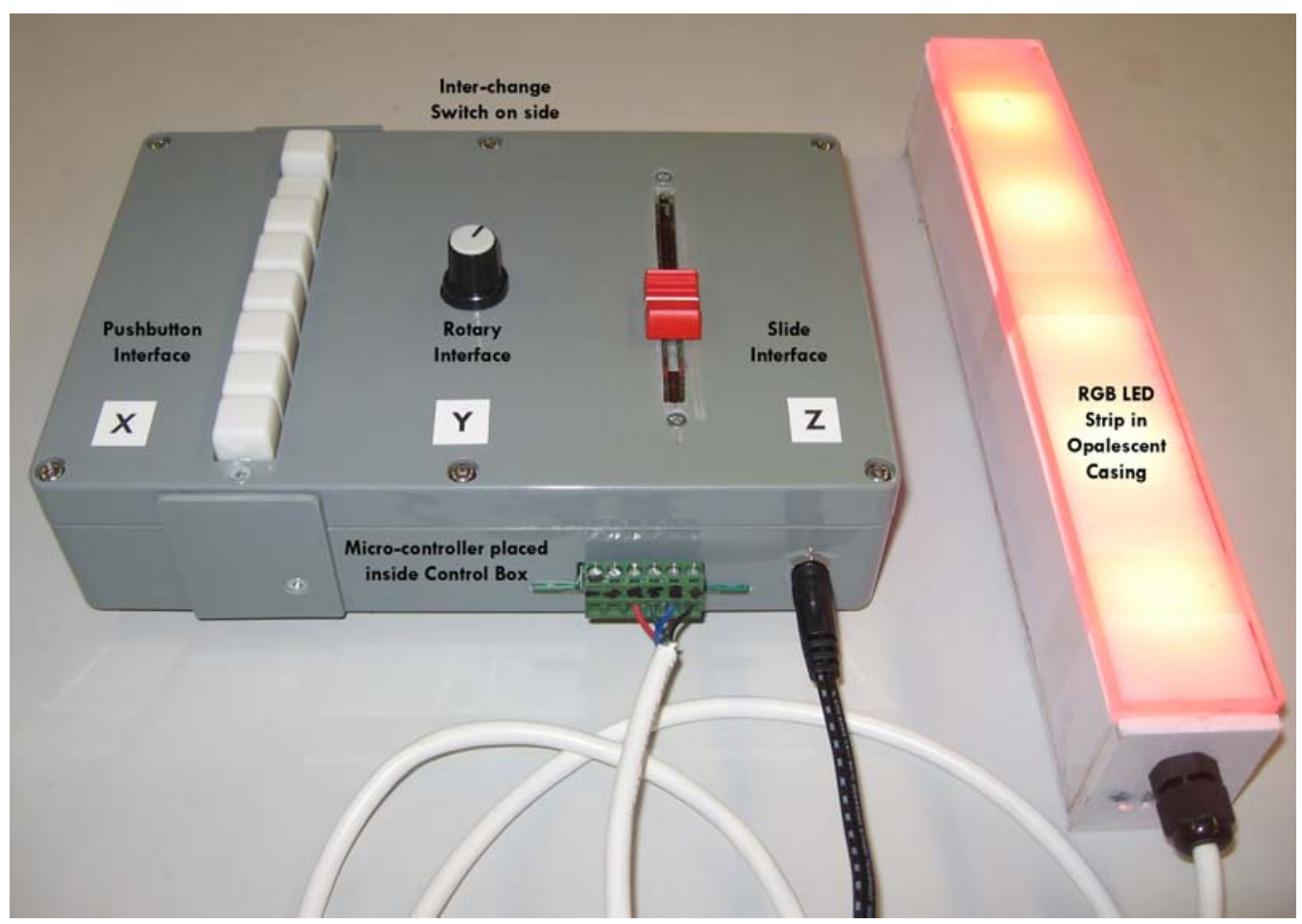

Figure 13 - Manual control interfaces for luminous colour [EI study: Eventual stage] 


\subsection{Execution: Eventual stage}

The eventual stage was executed within the premises of the Schools of Architecture and Design, Victoria University of Wellington in New Zealand. A brief demographic analysis of the thirty participants showed that eight are from the age group of 16-24 years, sixteen from the age group of 25-34 years, three from the age group of 35-44 years, two from the age group of 45-54 years, and one from the age group of 55 and above. Sixteen of the participants are male while fourteen are female.

\begin{tabular}{|c|c|c|c|c|}
\hline Demographics & \multicolumn{2}{|c|}{ India } & \multicolumn{2}{|c|}{ New Zealand } \\
\hline \multicolumn{5}{|l|}{ Age Group } \\
\hline $16-24$ & \multicolumn{2}{|c|}{24} & \multicolumn{2}{|c|}{8} \\
\hline $25-34$ & \multicolumn{2}{|c|}{1} & \multicolumn{2}{|c|}{16} \\
\hline $35-44$ & \multicolumn{2}{|c|}{2} & \multicolumn{2}{|c|}{3} \\
\hline $45-54$ & \multicolumn{2}{|c|}{2} & \multicolumn{2}{|c|}{2} \\
\hline $55 \&$ above & \multicolumn{2}{|c|}{1} & \multicolumn{2}{|c|}{1} \\
\hline \multicolumn{5}{|l|}{ Gender } \\
\hline Male & \multicolumn{2}{|c|}{8} & \multicolumn{2}{|c|}{16} \\
\hline Female & \multicolumn{2}{|c|}{22} & \multicolumn{2}{|c|}{14} \\
\hline \multicolumn{5}{|c|}{ Previous experience with } \\
\hline lighting controls & Yes & No & Yes & No \\
\hline Switching & 30 & 0 & 30 & 0 \\
\hline Dimming & 17 & 13 & 30 & 0 \\
\hline Preset Controllers & 4 & 26 & 7 & 23 \\
\hline
\end{tabular}

Table 8 - Summary of participant demographics from India and New Zealand [EI study]

All thirty participants have used lighting control interfaces before this study for turning on/off lights and controlling luminous intensity. The difference in socio-economic backgrounds between the two sample populations is quite evident, as the entire population in New Zealand has had previous experience with using interactive lighting controls prior to this experiment. However, only eight participants have used interfaces for controlling luminous colour while seven participants have used 
interfaces for recalling preset scenes. As a very few participants from both stages have used interfaces for luminous colour and preset control, it was assumed that the overall assessment of these interfaces would tend to be fair and unbiased, as most of them have used these interfaces for the first time. The following paragraphs describe the results of the eventual stage.

\subsubsection{Results - Evaluating interfaces}

The test scores for each of the operational definitions obtained from the quantitative data using inferential statistics are listed in Tables 9-12. Any other qualitative comments made by participants were coded, conceptualised and categorised using the grounded theory coding described in Section 5.5.3 under each of the operational definitions.

\subsubsection{Evaluating Interfaces - Appearance}

Participants rated the appearance of rotary and slide interfaces over the pushbutton interface as an interface for controlling luminous intensity. The percentage of favourable responses for the rotary interface was $90.0 \%$, the slide interface was $76.7 \%$, and the pushbutton interface was $23.3 \%$. These differences in percentages are highly significant as $p<0.001$.

Participants rated the appearance of the pushbutton interface over the rotary and slide interfaces as an interface for controlling luminous colours. The percentage of favourable responses for the pushbutton interface was $53.7 \%$, and both the rotary and slide interface was $26.7 \%$. These differences in percentages are significant as $p<0.05$. 


\subsubsection{Evaluating Interfaces - Accuracy}

Participants rated the pushbutton and slide interfaces as more accurate than the rotary interface for selecting their desired luminous intensity level. The mean rank for the pushbutton interface was 2.32, the slide interface was 2.30 , and the rotary interface was 1.38. These differences in mean ranks are highly significant as $p<0.001$.

Participants rated the pushbutton interface as more accurate than the slide and rotary interfaces for selecting their desired luminous colours. The mean rank for the pushbutton interface was 2.73 , the slide interface was 1.72 , and the rotary interface was 1.55. These differences in mean ranks are highly significant as $p<0.001$.

Participants rated the interface with a combination of iconic and textual representations of the depicted scenes as the most accurate, and the interface with numerical representations as the least accurate for recalling preset lighting scenes. The mean ranks for the five different interfaces were 4.40 for the combined textual and iconic representations of the scenes, 3.75 for purely textual, 3.62 for purely iconic, 1.68 for labelled scenes, and 1.55 for numerical representations. These differences in mean ranks are highly significant as $p<0.001$.

\subsubsection{Evaluating Interfaces - Learning speed}

Participants took the least amount of time to learn about the preset lighting scenes for recall from the interface with a combination of iconic and typographic representations of the depicted scenes, and most amount of time with the interface with only numerical representations. The mean ranks for the five different interfaces were 4.58 for the combined iconic and typographic, 3.80 for purely typographic, 3.38 for purely iconic, 1.67 for labelled scenes, and 1.57 for numerical 
representations of the scenes. These differences in mean ranks are highly significant as $p<0.001$.

\subsubsection{Evaluating Interfaces - Grabbability}

Participants rated the rotary interface as easier to grab than the slide and pushbutton interfaces while controlling luminous intensity. The percentage of favourable responses for the rotary interface was $96.7 \%$, the slide interface was $36.7 \%$, and the pushbutton interface was $26.7 \%$. These differences in percentages are highly significant as $p<0.001$.

\subsubsection{Evaluating Interfaces - Responsiveness}

Participants found no difference in the responsiveness of the pushbutton, rotary and slide interfaces for controlling luminous intensity. As $p>0.05$, it is accepted that the differences in mean ranks were due to random error.

Participants rated the slide and rotary interfaces as more responsive than the pushbutton interface for controlling luminous colours. The mean rank for the slide interface was 2.18, the rotary interface was 2.15 , and the pushbutton interface was 1.67. These differences in mean ranks are significant as $\mathrm{p}<0.05$.

\subsubsection{Evaluating Interfaces - Ease of use}

Participants rated the rotary interface as easier to use than the slide and pushbutton interfaces for controlling luminous intensity. The mean rank for the rotary interface was 2.82 , the slide interface was 1.73 , and the pushbutton interface was 1.45. These differences in mean ranks are highly significant as $p<0.001$. 
Participants rated the slide and rotary interfaces as easier to use than the pushbutton interface for controlling luminous colours. The mean rank for the slide interface was 2.32, rotary interface was 2.13, and the pushbutton interface was 1.55. These differences in mean ranks are significant as $p<0.05$.

Additionally, fifteen [50\%] participants commented that "rigid" or "stiff" interfaces that do not provide a "smooth" shift in gripping position are difficult to use and vice-versa.

\subsubsection{Evaluating Interfaces - Summary}

End-user evaluation of existing interfaces shows that participants in New Zealand were able to identify the function of luminous intensity control easily from the appearance of rotary interface in comparison to the slide interface and with difficulty from the pushbutton interface. They were able to select and repeat a selection of luminous intensity level accurately with both the pushbutton and slide interfaces, and the least amount of accuracy with the rotary interface. The larger dial of the rotary interface made it easier for the participants to grab in comparison to the smaller control handles of pushbutton and slide interfaces. Participants did not find any difference between the conversational interactive properties of these interfaces. However, they prefer using the rotary interface in comparison to the slide or pushbutton interfaces probably due the stiffness or rigidness of their control handles.

End-user evaluation of existing interfaces shows that participants in $\mathrm{New}$ Zealand were not able to identify the function of controlling luminous colour from the appearances of these interfaces. They were able to select and repeat a selection of luminous colour much more accurately 
with the pushbutton interface in comparison to the slide and rotary interfaces. The visibly perceptible movements of the control handles of the slide and rotary interfaces enabled participants to have a conversational type of interaction in comparison to the pushbutton interface. Participants found the slide interface easy to use, the rotary interface to be slightly less but easy nevertheless, and the pushbutton interface to be difficult to use probably due to the stiffness or rigidness of the buttons.

According to end-user evaluation of existing interfaces by participants in New Zealand, selection of desired lighting scenarios was quickest and most accurate with preset control interfaces that describe the preset scenes with a combination of text and images. Participants considered the interface with pure textual descriptions to be more accurate and required less time in comparison to the interface with pure images. Interfaces with labelled scenes and purely numerical representations were considered least accurate and required the highest amount of time for scene selection.

\begin{tabular}{|c|c|c|c|c|c|c|c|c|c|c|}
\hline \multirow[t]{2}{*}{ Type } & \multirow[t]{2}{*}{ Dimension } & \multicolumn{3}{|c|}{ Pushbutton } & \multicolumn{3}{|c|}{ Rotary } & \multicolumn{3}{|c|}{ Slider } \\
\hline & & $M$ & $S D$ & $\begin{array}{l}\text { Min- } \\
\text { Max }\end{array}$ & M & $S D$ & $\begin{array}{l}\text { Min- } \\
\text { Max }\end{array}$ & $M$ & $S D$ & $\begin{array}{l}\text { Min- } \\
\text { Max }\end{array}$ \\
\hline \multirow{5}{*}{ 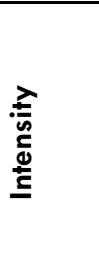 } & Appearance & 1.233 & 0.430 & $1-2$ & 1.900 & 0.305 & $1-2$ & 1.767 & 0.430 & $1-2$ \\
\hline & Accuracy & 2.533 & 0.776 & $1-3$ & 1.733 & 0.640 & $1-3$ & 2.533 & 0.629 & $1-3$ \\
\hline & Grabbability & 1.267 & 0.450 & $1-2$ & 1.967 & 0.183 & $1-2$ & 1.367 & 0.450 & $1-2$ \\
\hline & Responsiveness & 2.467 & 0.629 & $1-3$ & 2.600 & 0.675 & $1-3$ & 2.267 & 0.640 & $1-3$ \\
\hline & Ease of Use & 2.400 & 0.814 & $1-4$ & 3.833 & 0.379 & $3-4$ & 2.733 & 0.640 & $2-4$ \\
\hline \multirow{4}{*}{$\frac{3}{0}$} & Appearance & 1.533 & 0.507 & $1-2$ & 1.267 & 0.450 & $1-2$ & 1.267 & 0.450 & $1-2$ \\
\hline & Accuracy & 2.900 & 0.305 & $2-3$ & 1.933 & 0.583 & $1-3$ & 2.067 & 0.583 & $1-3$ \\
\hline & Responsiveness & 1.933 & 0.868 & $1-3$ & 2.467 & 0.681 & $1-3$ & 2.500 & 0.682 & $1-3$ \\
\hline & Ease of Use & 2.567 & 0.935 & $1-4$ & 3.233 & 0.898 & $1-4$ & 3.400 & 0.770 & $1-4$ \\
\hline
\end{tabular}

Table 9 - Summary of Means, Standard Deviations and Minimum-Maximum range for the different luminous intensity and colour control interfaces [EI study: Eventual stage] 


\begin{tabular}{|c|c|c|c|c|c|c|}
\hline \multirow[t]{2}{*}{ Type } & \multirow[t]{2}{*}{ Dimension } & Pushbutton & \multicolumn{2}{|l|}{ Rotary } & & \\
\hline & & \multicolumn{3}{|c|}{$\begin{array}{l}\text { (Does not look like) Looks like \% } \\
\text { Or (Hard to grab) Easy to grab \% }\end{array}$} & $p$ & $\begin{array}{l}\text { Cochran } \\
Q_{(d f=2)}\end{array}$ \\
\hline \multirow[t]{2}{*}{ Intensity } & Appearance & $(76.7) 23.3$ & $(10.0) 90.0$ & (23.3) 76.7 & $<0.001$ & 25.846 \\
\hline & Grabbability & (73.3) 26.7 & (3.3) 96.7 & (63.3) 36.7 & $<0.001$ & 26.690 \\
\hline \multirow[t]{2}{*}{ Colour } & Appearance & (46.3) 53.7 & $(73.3) 26.7$ & (73.3) 26.7 & 0.023 & 7.529 \\
\hline & & \multicolumn{3}{|c|}{ Mean ranks } & $p$ & $\chi^{2}(d f=2)$ \\
\hline \multirow[t]{3}{*}{ Intensity } & Accuracy & 2.32 & 1.38 & 2.30 & $<0.001$ & 20.337 \\
\hline & Responsiveness & 2.00 & 2.22 & 1.78 & 0.089 & 4.829 \\
\hline & Ease of Use & 1.45 & 2.82 & 1.73 & $<0.001$ & 36.725 \\
\hline \multirow[t]{3}{*}{ Colour } & Accuracy & 2.73 & 1.55 & 1.72 & $<0.001$ & 35.167 \\
\hline & Responsiveness & 1.67 & 2.15 & 2.18 & 0.003 & 11.577 \\
\hline & Ease of Use & 1.55 & 2.13 & 2.32 & 0.002 & 12.822 \\
\hline
\end{tabular}

Table 10 - Percentages of responses and mean ranks for the different luminous intensity and colour control interfaces [El study: Eventual stage]

\begin{tabular}{|c|c|c|c|c|c|c|c|}
\hline Type & Dimension & & $\begin{array}{c}\text { Numbers } \\
\text { only }\end{array}$ & $\begin{array}{l}\text { Text } \\
\text { only }\end{array}$ & $\begin{array}{l}\text { Icons } \\
\text { only }\end{array}$ & Labels & $\begin{array}{l}\text { Text + } \\
\text { lcons }\end{array}$ \\
\hline \multirow{6}{*}{$\begin{array}{l}\bar{\Phi} \\
\Phi \\
\Phi \\
\vdots\end{array}$} & \multirow[t]{3}{*}{ Accuracy } & Mean & 1.067 & 2.500 & 2.400 & 1.167 & 2.933 \\
\hline & & $S D$ & 0.365 & 0.509 & 0.675 & 0.461 & 0.254 \\
\hline & & Min-Max & $1-3$ & $2-3$ & $1-3$ & $1-3$ & $2-3$ \\
\hline & \multirow[t]{3}{*}{ Learning Speed } & Mean & 1.067 & 2.467 & 2.167 & 1.133 & 2.933 \\
\hline & & $S D$ & 0.254 & 0.571 & 0.592 & 0.346 & 0.254 \\
\hline & & Min-Max & $1-2$ & $1-3$ & $1-3$ & $1-3$ & $2-3$ \\
\hline
\end{tabular}

Table 11 - Summary of Means, Standard Deviations and Minimum-Maximum range for the different preset scene control interfaces [El study: Eventual stage]

\begin{tabular}{|c|c|c|c|c|c|c|c|c|}
\hline Type & Dimensions & $\begin{array}{c}\text { Numbers } \\
\text { only }\end{array}$ & $\begin{array}{l}\text { Text } \\
\text { only }\end{array}$ & $\begin{array}{l}\text { Icons } \\
\text { only }\end{array}$ & Labels & $\begin{array}{l}\text { Text + } \\
\text { lcons }\end{array}$ & & \\
\hline \multirow[b]{3}{*}{ Preset } & & \multicolumn{5}{|c|}{ Mean Ranks } & $p$ & $\chi^{2}(d f=4)$ \\
\hline & Accuracy & 1.55 & 3.75 & 3.62 & 1.68 & 4.40 & $<0.001$ & 98.618 \\
\hline & $\begin{array}{l}\text { Learning } \\
\text { Speed }\end{array}$ & 1.57 & 3.80 & 3.38 & 1.67 & 4.58 & $<0.001$ & 100.016 \\
\hline
\end{tabular}

Table 12 - Mean ranks for the different preset scene control interfaces [El study: Eventual stage]

\subsubsection{Results - Designing interfaces}

The qualitative data in the form of design suggestions made by participants are coded, conceptualised and categorised using the 
grounded theory coding described in Section 5.5.3 under two categories for designing interfaces: better existing and future tangible. The system used is to list all suggestions made by participants in terms of continuous prose, listed answers, or conceptual sketches. These are then quantified by grouping all similar descriptions under the number of participants who made these suggestions in Table 13.

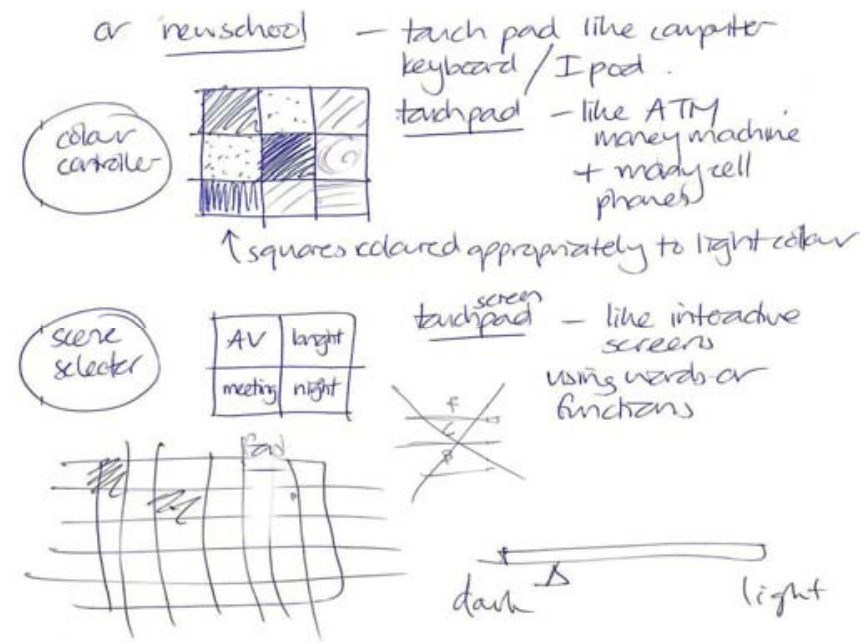

Figure 14 - Design suggestions for future tangible interfaces [El study: Eventual stage]

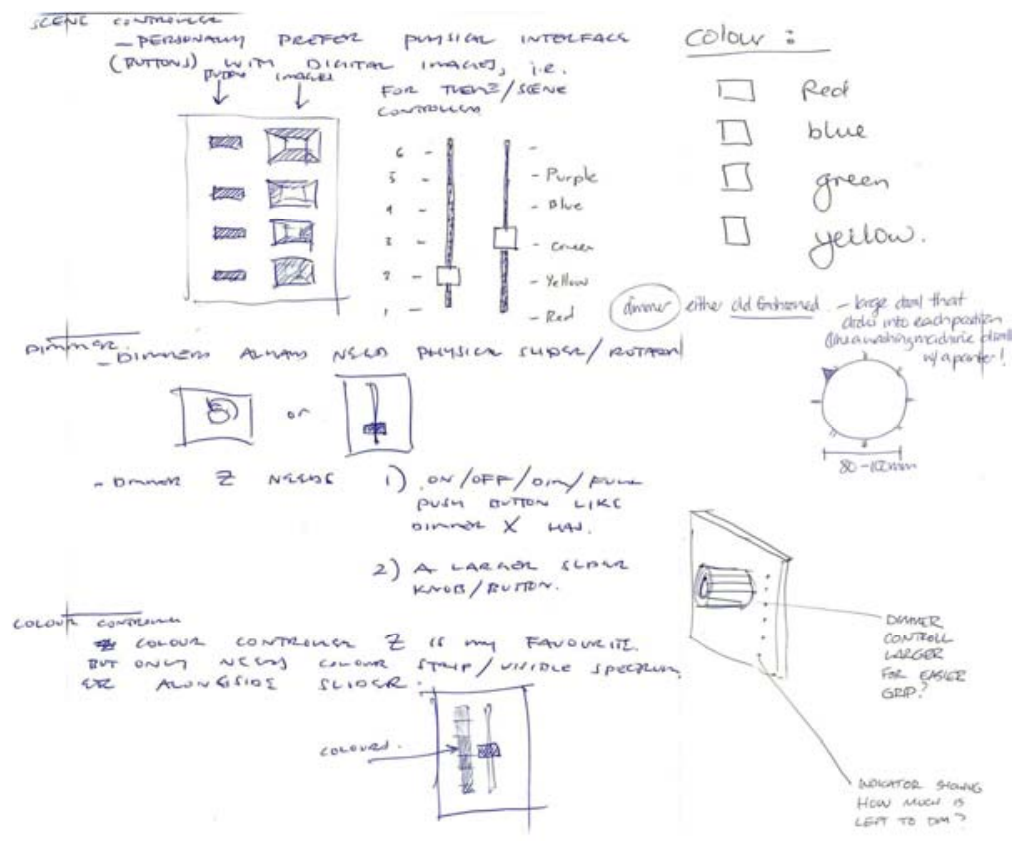

Figure 15 - Design suggestions for better existing interfaces [EI study: Eventual stage] 


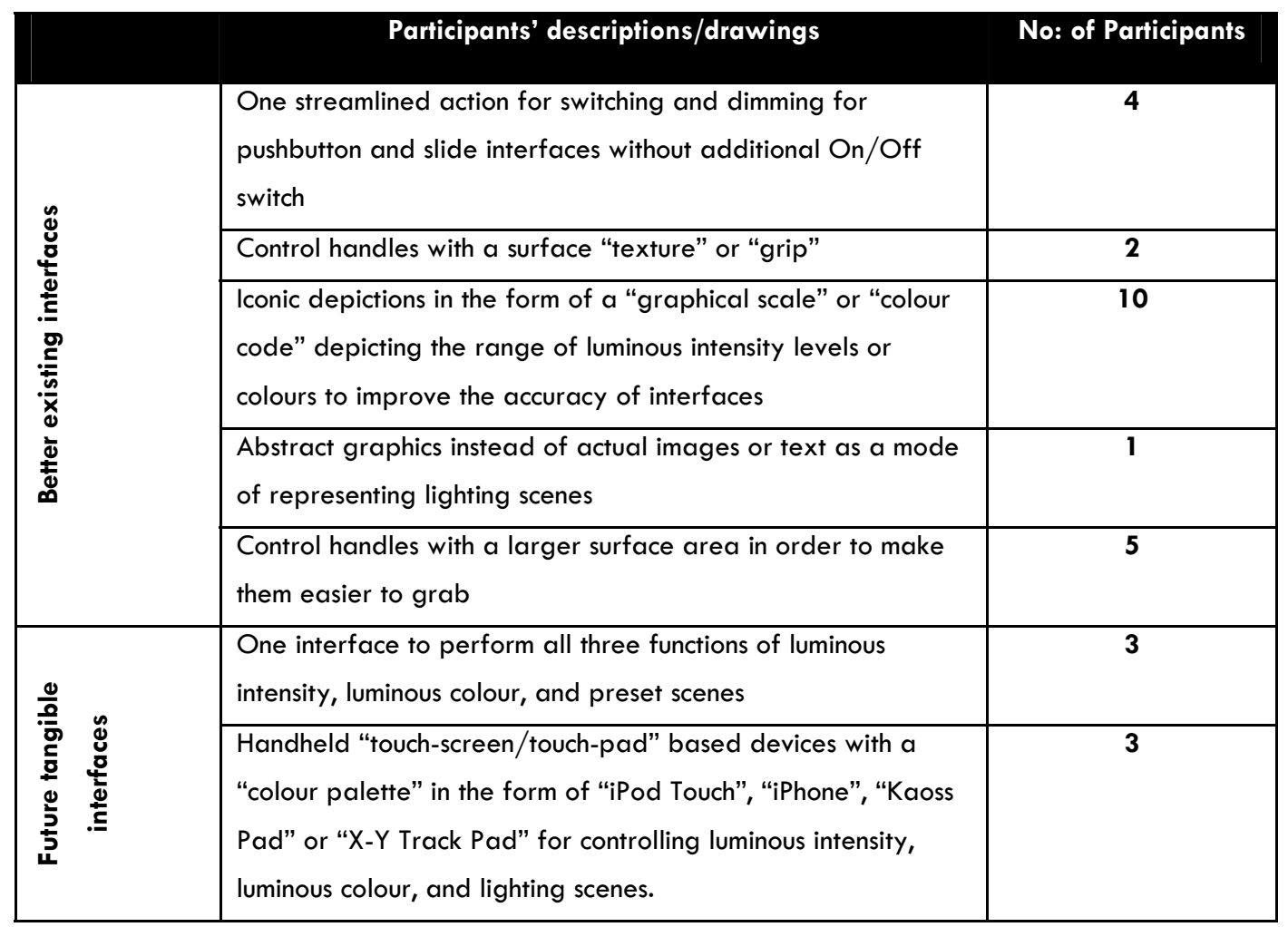

Table 13 - Summary of participants' suggestions for most desirable interface designs [El study: Eventual stage]

\subsection{Compilation: El study}

The results of the pilot and eventual stages were analysed and compared to test hypotheses, or combined to generate hypotheses for designing better existing and future tangible interfaces. The test scores and qualitative comments for each of the operational definitions were used as a means of comparing the results between the two stages and arrive at probable reasons for the differences in test scores. All similar design suggestions made by participants from the two stages were combined to generate a new hypothesis about future tangible interfaces for future testing as described in Section 5.5.3. 


\begin{tabular}{|c|c|c|}
\hline Interface type & Illustration & Participants comments about existing interfaces \\
\hline $\begin{array}{l}\text { Type: Pushbutton } \\
\text { Manufacturer: Lutron } \\
\text { Model: Rania } \\
\text { Source: } \\
\text { http://www.lutron.com }\end{array}$ & 1 & $\begin{array}{l}\text { Pros: } \\
\text { - Enables accurate selection } \\
\text { Cons: } \\
\text { - Does not afford dimming } \\
\text { - Not easy to grasp in the hands } \\
\text { - Inadequate visual feedback } \\
\text { - Stiff or rigid }\end{array}$ \\
\hline $\begin{array}{l}\text { Type: Rotary dimmer } \\
\text { Manufacturer: Anchor } \\
\text { Model: Rotary }\end{array}$ & (2) & $\begin{array}{l}\text { Pros: } \\
\text { - Affords dimming [for participants of the } \\
\text { eventual stage] } \\
\text { - Easy to grasp in the hands } \\
\text { - Smooth or fluid } \\
\text { Cons: } \\
\text { - Does not afford dimming [for participants of the } \\
\text { pilot stage] } \\
\text { - Does not enable accurate selection } \\
\text { - Inadequate visual feedback }\end{array}$ \\
\hline $\begin{array}{l}\text { Type: Slide dimmer } \\
\text { Manufacturer: Lutron } \\
\text { Model: Lyneo } \\
\text { Source: } \\
\text { http://www.lutron.com }\end{array}$ & $\begin{array}{l}1 \\
1 \\
1 \\
\end{array}$ & $\begin{array}{l}\text { Pros: } \\
\text { - Affords dimming } \\
\text { - Enables accurate selection } \\
\text { Cons: } \\
\text { - Inadequate visual feedback } \\
\text { - Not easy to grasp in the hands } \\
\text { - Stiff or rigid }\end{array}$ \\
\hline
\end{tabular}

Table 14 - Analysis of participant preferences from the existing interfaces

Majority [ $>$ or $=80 \%$ ] of participants from both samples are between the age group of 16-34. The ratio of male-female participants from India and New Zealand are 1:2.5 and 1.143:1 respectively. The difference in socio-economic backgrounds between the two sample populations is quite evident, as $100 \%$ of the population from New Zealand has had previous experience with using dimming control while only $56.67 \%$ of the population from India have used them prior to this experiment. However, as very few participants $[<$ or $=23.3 \%$ ] from 
both samples have used interfaces for preset control, it was assumed that the overall assessment of these interfaces would tend to be fair and unbiased, as most of them have used these interfaces for the first time. Participant demographics are listed in Table 8.

An overall analysis of the results of the two stages in Table 13 shows that the study has high construct, internal and external validity as described in Sections 5.6.1, 5.6.2 and 5.6.3 respectively. The only notable differences between the test scores of the two stages are in the appearance of the rotary interface for luminous intensity control and the responsiveness $p$-values of the luminous colour control interfaces. The appearance of the rotary interface can be accounted to the socioeconomic backgrounds of the sample populations, as participants in India are familiar with a different function for the rotary interface. The difference in responsiveness $p$-values can be accounted to the fact that a new set of interfaces with visibly perceptible movements were used for controlling luminous colour in the eventual stage.

\subsubsection{Analysis - Test hypotheses}

The test scores provide quantitative evidence, while participant comments provide qualitative evidence for supporting and verifying the hypotheses.

\subsubsection{Hypothesis \# 1}

- Physical designs of rotary and slide interfaces enable quicker recognition and more effective operation of the luminous intensity control function than pushbutton interfaces.

The test scores from the pilot and eventual stages provide adequate evidence to show that rotary and slide interfaces enable quicker 
recognition and more effective operation of the luminous intensity control function than pushbutton interfaces. Interestingly one participant from the eventual stage even commented that the pushbutton interface is "a typical case of forcing new design on us!" Therefore this hypothesis is adequately supported.

Although the physical design of rotary interface for luminous intensity control received the highest percentage of favourable response with a high significance level in the eventual stage, the test scores from the pilot stage show a low percentage of favourable response with a low significance level. This result can be accounted to the fact that participants in India are more accustomed to using the rotary interface as a speed regulator for fans than for dimming lights. This in turn shows that geographical and cultural aspects play a significant role in end-users' subjective perception.

- Physical designs of pushbutton interfaces enable quicker recognition and more effective operation of the luminous colour control function than rotary and slide interfaces.

The test scores from the pilot and eventual stages do not provide adequate evidence to show that the physical designs of pushbutton interfaces enable quicker recognition and effective operation of the luminous colour control function than rotary and slide interfaces. Although the physical design of the pushbutton interface received a slightly higher percentage of favourable response with a low significance level in the eventual stage, the test scores from the pilot stage show the difference in percentages of responses was due to random error. Therefore this hypothesis is not supported. 


\subsubsection{Hypothesis \# 2}

- Expressive iconographic and typographic representations of luminous intensities/colours enable quicker recognition and more accurate selection of the desired luminous intensities/colours than no representations.

The test scores and design suggestions from the pilot and eventual stages provide some evidence to show that expressive iconographic and typographic representations of luminous intensities/colours enable quicker recognition and more accurate selection of the desired luminous intensities/colours than no representations. The pushbutton interface for luminous intensity control is the only interface that depicts the accurate luminous intensity level selected in the form of green LED display, and scored the highest mean rank in both the stages, although at different significance levels. Four participants from the pilot stage and ten participants from the eventual stage have even suggested their desire for expressive icons and text depicting the range of luminous intensity levels or colours for improving the accuracy of interfaces. Therefore this hypothesis requires further testing in the $\mathrm{Tl}$ study.

- Expressive iconographic and typographic representations of lighting layers/scenes enable quicker recognition and more accurate selection of the desired lighting layers/scenes than representations with individual alphanumeric characters.

The test scores from the pilot and eventual stages provide adequate evidence to show that expressive iconographic and typographic representations of lighting scenes enable quicker recognition and more accurate selection of the desired lighting scenes than representations with individual alphanumeric characters. The interface with a combination of iconic and typographic representations of the depicted scenes scored the highest mean ranks whereas the interface with only numerical 
representations scored the lowest mean ranks for learning speed and accuracy at high significance levels. Therefore this hypothesis is adequately supported.

\subsubsection{Hypothesis \# 3}

- Interface control handles with larger surface areas are more acceptable than control handles with small surface areas.

The test scores from the pilot and eventual stages provide adequate evidence to show that interface control handles with larger surface areas are more acceptable than control handles with small surface areas. The rotary dial for controlling luminous intensity has the largest surface area in comparison to the slider handle and pushbuttons, and has received the highest percentage of favourable responses [ $>85 \%$ ] in both the stages for grabbability, although at different significance levels. Five participants from the eventual stage have even expressed their desire for control handles with larger surface areas. Therefore this hypothesis is adequately supported.

- Interface control handles with textured gripping surfaces are more acceptable than control handles without these surfaces.

Design suggestions from the pilot and eventual stages provide some evidence to show that interface control handles with textured gripping surfaces is more acceptable than control handles without these surfaces. One participant from the pilot stage and two participants from the eventual stage have expressed their desire for control handles with textured gripping surfaces. Therefore this hypothesis is partially supported. 


\subsubsection{Hypothesis \# 4}

- Interface control handles that provide an effortless shift in the gripping position are more acceptable than interfaces that do not provide an effortless shift.

Participant comments and test scores from the pilot and eventual stages provide adequate evidence to show that interface control handles that provide an effortless shift in the gripping position are more acceptable than interfaces that do not provide an effortless shift. Six participants from the pilot stage and fifteen participants from the eventual stage have commented that rigid or stiff interfaces are difficult to use. Additionally, rotary interfaces for controlling luminous intensity and colour, and slide interface for controlling luminous colour are interfaces that apparently provide an effortless shift in gripping position and thereby scored the highest mean ranks in ease of use, although at different significance levels. Therefore this hypothesis is adequately supported.

\subsubsection{Hypothesis \# 5}

- Interfaces that provide noticeable visual changes during manipulation are considered more responsive than interfaces that do not provide these changes.

The test scores from the pilot and eventual stages do not provide adequate evidence to show that interfaces that provide noticeable visual changes during manipulation are considered more responsive than interfaces that do not provide these changes. Participants do not find any difference in the responsiveness of interfaces used for controlling luminous intensity as their test scores show that the difference in mean ranks was due to random error. Similarly, participants do not find any difference in the responsiveness of interfaces used for controlling luminous colour in the pilot stage. However, the slide and rotary interfaces used 
for controlling luminous colour in the eventual stage scored higher mean ranks than the pushbutton interface. Apparently, both these interfaces provide noticeable visual responses upon manipulation. The slide interface used in the eventual stage has a much longer slider length showing a noticeable change in position than the one used in the pilot stage. The rotary interface used in the eventual stage has an indicative pointer showing a noticeable change in position as compared to the one used in the pilot stage, which does not have such a pointer. Therefore this hypothesis requires further testing in the Tl study.

\subsubsection{Analysis - Generate new hypotheses}

The hypotheses about designing better existing and future tangible interfaces are generated from the design suggestions made by participants from the pilot and eventual stages. The following are the most prominent suggestions made by participants from both stages:

- One streamlined action to perform the two functions of switching and dimming.

- One interface that performs all the lighting control functions of controlling luminous intensities/colours and programming/recalling lighting scenes.

- Lighting control interface that resembles and performs the activities of everyday objects such as iPods or iPhones.

- Touch-screen graphical interface that provides iconographic representations of luminous intensities/colour as well as lighting layers/scenes. 


\subsubsection{Hypothesis \# 7}

End-users prefer an interface that represents and maps the lit environment in a manner that gives the impression of direct interaction with, and control of, the available lighting layers.

\subsection{Chapter Inference}

Chapter 6 has described the implemented procedure and results of the EI study. The pilot and eventual stages of this study were conducted with participants from two different schools of architecture in India and New Zealand respectively. Three different types of physical interfaces namely pushbutton, rotary and slider were used for the manual control of luminous intensity and colour. The interfaces for controlling luminous intensity were procured from the local market, while interfaces for controlling luminous colour were custom fabricated. Five different types of screen-based virtual interfaces were custom fabricated for the recall of preset lighting scenes. The preset scenes were depicted with five different combinations of individual and collective iconographic and typographic representations.

The analysis of the quantitative and qualitative data obtained from both the stages shows that existing designs of manual dimming and preset control interfaces either convey partial or no information at all about the different lighting scenarios they are intended to create and how endusers can use them. Quicker recognition and more effective operation is facilitated by the physical designs of rotary and slide interfaces for controlling luminous intensity control, and to some extent by the physical designs of pushbutton interfaces for controlling luminous colour. However, all three types of existing interfaces require information that is richly 
represented in the form of expressive iconography and typography for maximising the speed of recognition and the accuracy of selection. Similarly, interfaces for preset control require information that is richly represented in the form of expressive iconography and typography for facilitating quicker recognition and more accurate selection of the preset scenes. Existing interfaces for preset control use alphanumeric characters for representing the different preset lighting scenes for recall, which do not convey any information about the scenes.

Control handles that provide rich tactile responses with large surface area and texture can lead to end-users experiencing the graspable and material properties of interfaces. Conversational properties of interfaces can be experienced when the interface provides rich visual response with noticeable visual changes upon manipulation. Existing interfaces for manual dimming control do not provide the rich tactile or visual responses required for end-users to experience their interactive property during explorative manipulation. The analysis of the design suggestions obtained from both the stages describes end-user desire for handheld remote interfaces that provide the option of controlling touch-screen based luminous iconographic representations of luminous intensities/colour and lighting scenes. Finally, these results also provide evidence to suggest that the key characteristics of tangible lighting control interfaces can be used as evaluation tools as this study has high construct, internal and external validity.

In Chapter 6, implementation of the El study for observing and documenting end-user behaviour with existing interfaces for lighting control has been described. The documented results on end-user evaluation of these interfaces are used to test hypotheses as well as generate new hypotheses and design ideas about future tangible 
interfaces. The design ideas and hypotheses relating to these interfaces have to be tested in the $\mathrm{Tl}$ study. Chapter 7 will describe the implemented procedure and results of the TI study. 


\section{CHAPTER SEVEN}

\section{Evaluating Virtual Prototypes}

\subsection{Chapter Intent}

Chapter 7 describes the portion of the interactive studies involving the design and end-user evaluation of prototype existing and future tangible interfaces for lighting control. In Chapter 6, results of the Existing interface [EI] study have been used to support the first five hypothetical requirements for end-user interface with personalised lighting control from Chapter 3, as well as generate new hypotheses. The Tangible interface $[\mathrm{TI}]$ study has to be activated to enable end-user evaluation of the usability and end-user experience of future tangible interfaces. This involves formulating testable hypotheses from a combination of the hypotheses from Chapters 3 and 6 , and selecting participants. The execution of the study first requires construction of a test lit environment with different layers of lighting, and fabrication of virtual prototypes of existing and future tangible interfaces for controlling this environment. Once the interfaces are fabricated, the study will have to be executed by enabling participants to use the prototypes and evaluate them. The results obtained from this study will have to be analysed and interpreted to support the remaining and overall hypotheses of this thesis research. The outlook is towards meeting the overall aims of promoting end-user interaction with lit environments through tangible lighting control interfaces and understanding the real needs of end-users. 
Section 7.2 formulates testable hypotheses, and selects participants for the $\mathrm{Tl}$ study. Section 7.3 describes the methods used for constructing the test lit environment for lighting control. Section 7.4 describes the process of designing and fabricating virtual prototypes of an existing interface and a future tangible interface for controlling this environment. Section 7.5 describes the executed procedure and results of this study. Section 7.6 analyses and interprets the results of the TI study. Section 7.7 then summarises Chapter 7, and draws inferences for reviewing the project in Chapter 8 and discussing future directions in Chapter 9.

\subsection{Activation: Tl study}

The primary step for activating the TI study is to formulate testable hypotheses from the hypothetical requirements for end-user interface with personalised lighting controls described in Chapter 3 and the new hypotheses from the El study in Chapter 6. Participants will then have to be selected for the execution of this study.

\subsubsection{Formulation - Hypotheses}

In the following paragraphs, the hypotheses describing end-user requirements for recognition, exploration and reliance along with the new hypotheses from the El study are turned into statements that can be tested and verified in quantitative and qualitative terms. Additionally, those hypotheses that have not gathered adequate evidence from the El study are also listed. The differences between the percentages of favourable responses or mean ranks, and the p-value of tests will determine the significance of these hypotheses. 


\subsubsection{Hypothesis \# 2}

End-users learn to use a lighting control interface quickly and effectively when the iconography and typography depicted on the interface richly represents luminous colours or intensities, and lighting layers or scenes.

- Expressive iconographic and typographic representations of luminous intensities/colours enable quicker recognition and more accurate selection of the desired luminous intensities/colours than no representations.

\subsubsection{Hypothesis \# 5}

End-users experience the conversational property of an interface when it provides rich visual responses by a synchronic visual link to the changes in spatial illumination.

- Interfaces that provide noticeable visual changes during manipulation are considered more responsive than interfaces that do not provide these changes.

\subsubsection{Hypothesis \# 6}

End-users perform the different lighting control functions quickly and effectively when the lit environment and the interface become a single entity while achieving the desired lit scenario.

- Interfaces that represent and map the lit environment in a manner that gives the impression of direct interaction with, and control of, the available lighting layers enable quicker recognition and more effective operation of the luminous intensity/colour and preset control functions than interfaces that do not map these layers. 


\subsubsection{Hypothesis \# 7}

End-users prefer an interface that represents and maps the lit environment in a manner that gives the impression of direct interaction with, and control of, the available lighting layers.

- Interfaces that represent and map the lit environment in a manner that gives the impression of direct interaction with, and control of, the available lighting layers are more acceptable than interfaces that do not map these layers.

\subsubsection{Selection - Participants}

The different departments at Victoria University of Wellington in New Zealand were the target location for sampling population for the TI study. A list of all the students and staff members from the different departments was made and forty participants were approached randomly from this list. Participants from different departments were approached for this study in order to sample a population with characteristics from various other backgrounds apart from architecture and design. Participants showed a high level of interest for participation in the study as thirty-six out of forty [90\%] participants approached agreed to participate.

\subsection{Construction: Test environment}

The test environment was built to enable participants to navigate, select, group and switch or dim their desired layers of lighting for programming and recalling desired lighting scenarios. However given the time and resource constraints, a virtual immersive environment was considered for conducting the experiment. Additionally, the principle of contextual 
experiences and concept of an interactivated space from Section 4.4.1 were also considered while building the virtual environment. A virtual environment first required photographing all possible permutations and combinations of lighting scenarios in a real lit environment. Dr. Stuart Marshall from the School of Engineering and Computer Science at Victoria University of Wellington, New Zealand then used these photographs to develop a web-server using Java, which allows the projection of these photographs on a screen giving the impression of a configurable-lit environment.

\subsubsection{Test Environment - Real}

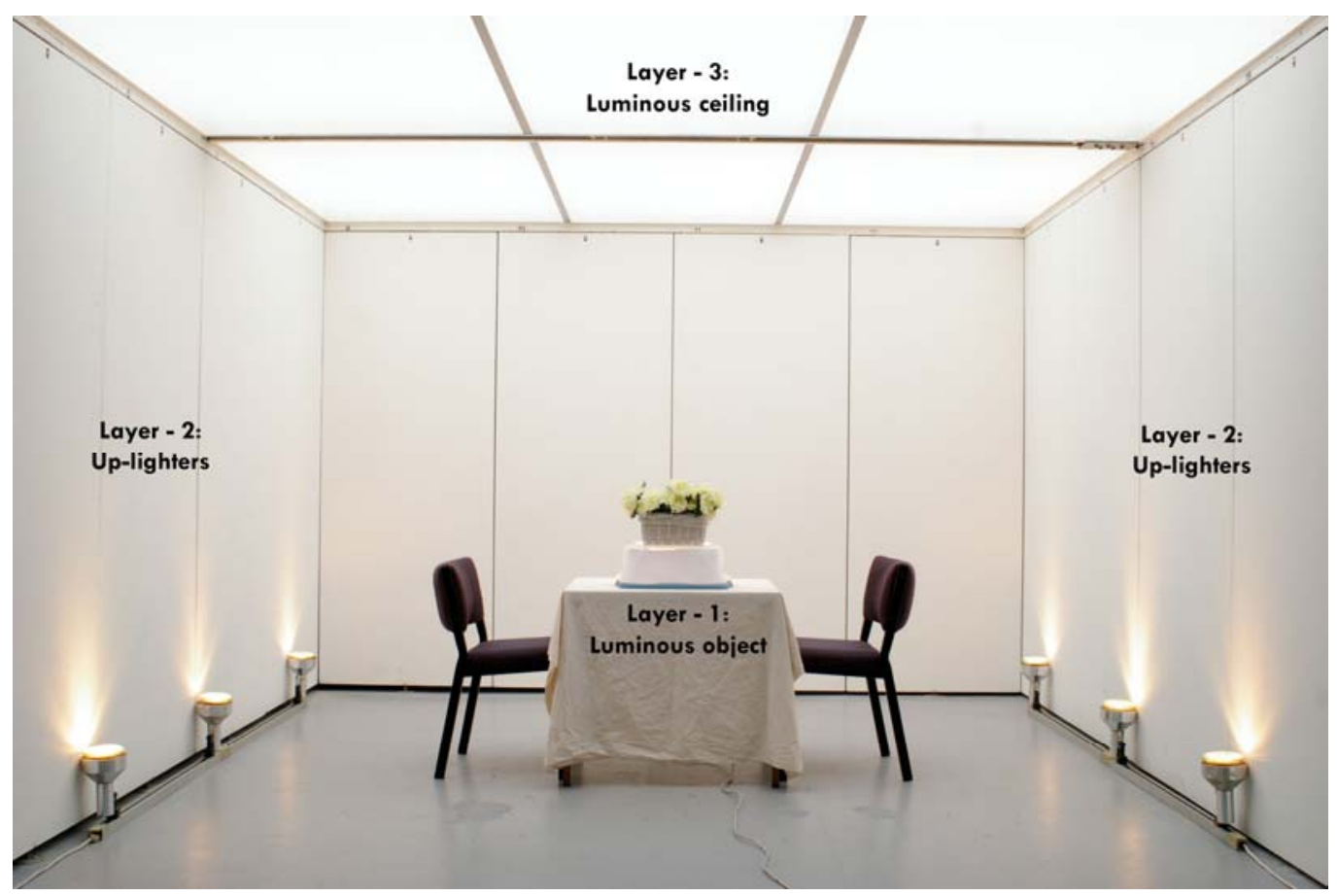

Figure 16 - Test Environment [Real]

The test environment for photographing different lighting scenarios was setup in the lighting laboratory of the Schools of Architecture and Design at Victoria University of Wellington in New Zealand. The setup consists of a cubicle with rotatable walls, which are painted white on one side. 
Inside the cubicle, the scene was set as a table for two. A luminous object placed at the centre of the table, backlit with three $40 \mathrm{~W}$ A-type lamps formed 'Layer - 1' of the test environment. The two sidewalls of the cubicle, up-lit with three spotlights of $120 \mathrm{~W}$ PAR38 lamps mounted on tracks placed on the floor alongside each wall formed 'Layer - 2' of the test environment. The luminous ceiling of the cubicle, backlit with a battery of 54W T16 coloured fluorescent lamps formed 'Layer - 3' of the test environment. A basket of flowers was placed on top of Layer 1 , to make the task of selecting a lighting scenario meaningful from the participants' perspective.
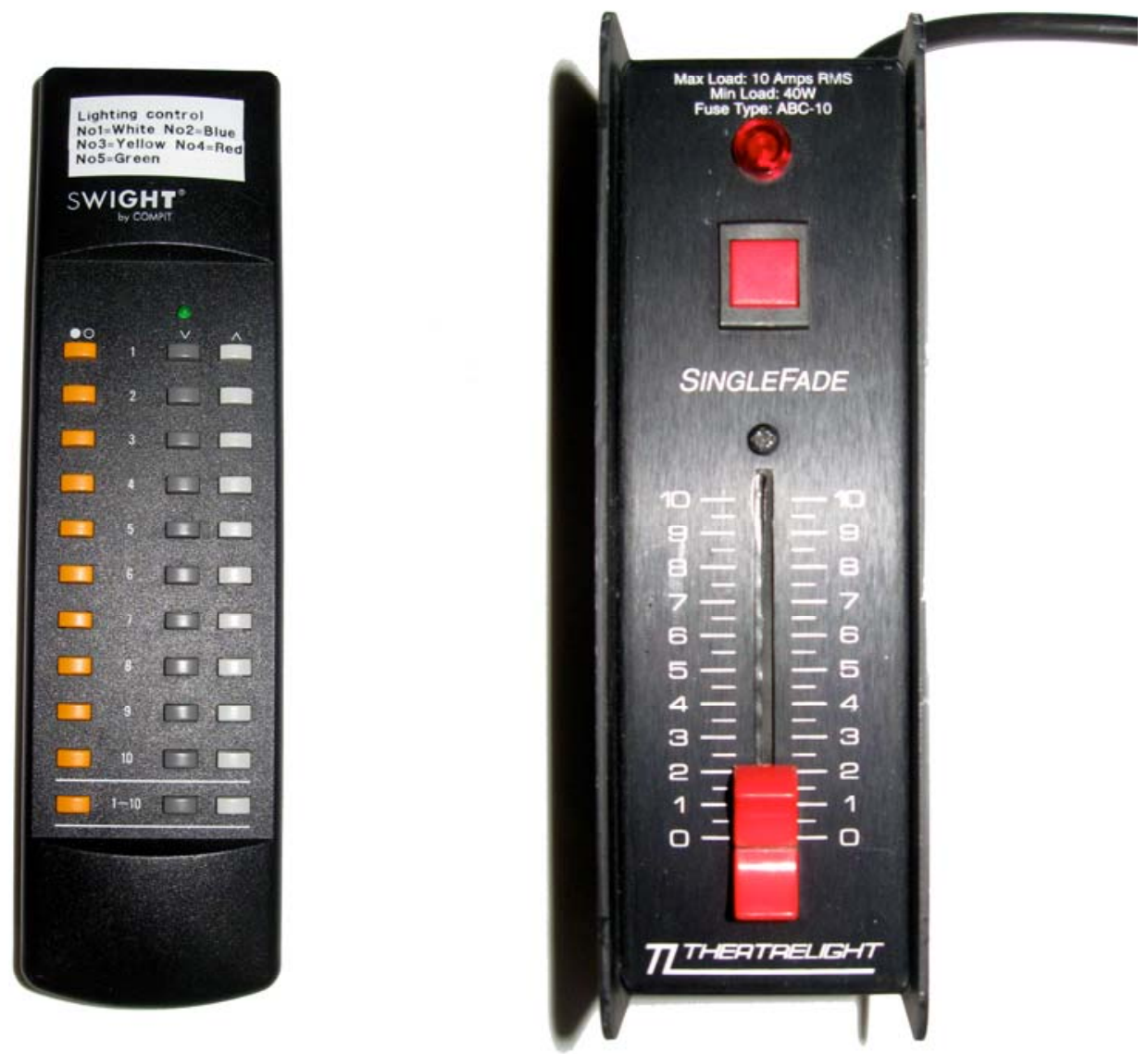

Figure 17 - IR remote control to select luminous colours from Layer - 3, and SingleFade dimmer to select luminous intensities from Layers -1 and -2 of the Test Environment [Real]. 
Calibrated manual 'SingleFade' standalone portable digital dimmers from the manufacturer Theatre Light NZ were used to select different luminous intensity levels from Layers -1 and -2 . An IR remote control was used to select five different luminous colours inside the cubicle from Layer - 3 namely amber, blue, green, red and white. During the process of photographing the different lighting scenarios, it was observed that the only perceivable difference in the brightness of Layers -1 and -2 in photographs was at dimmer calibration levels of $0 \%, 50 \%, 75 \%$ and $100 \%$. Therefore photographs of lighting scenarios with only these luminous intensity levels were taken for the virtual environment. For example, the first scene is photographed at brightness levels of $0 \%$ for Layers -1 and -2 , and a luminous colour of red for Layer -3 , while the next scene is photographed with Layer - 1 at $0 \%$, Layer -2 at $50 \%$ and Layer -3 at red, and so on.

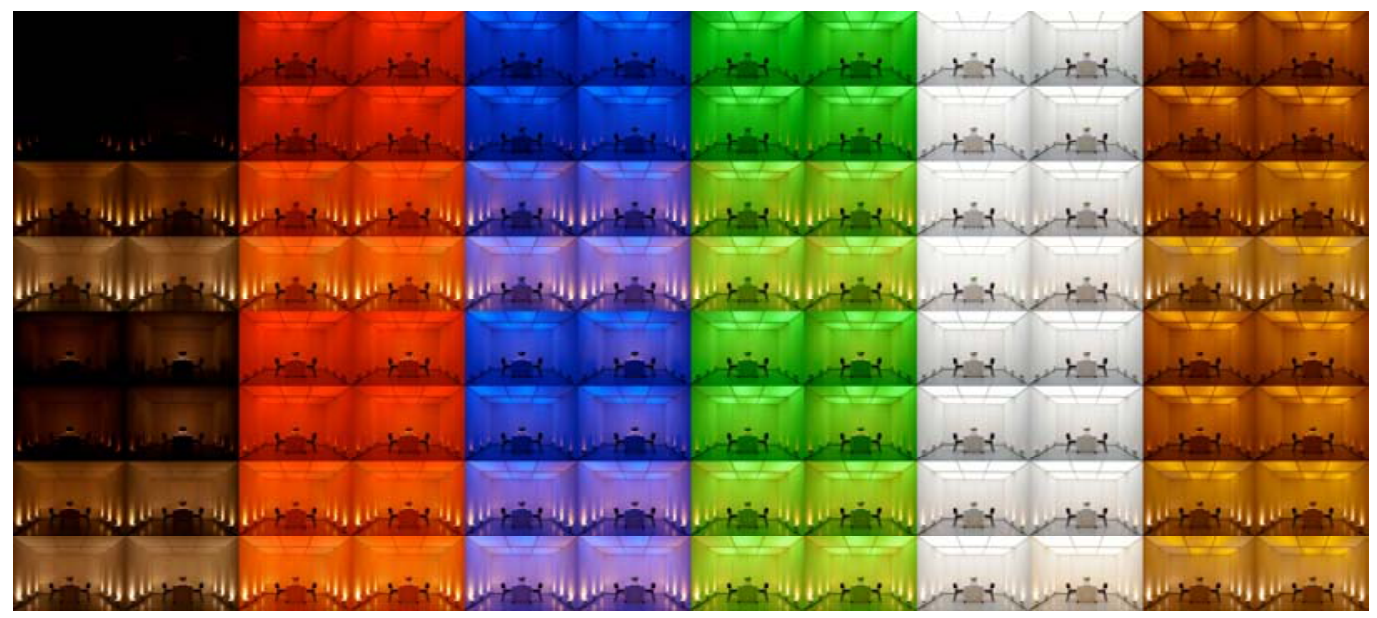

Figure 18 - 6 × 16 scenes of the test environment

The total number of photographs for combinations under each luminous colour of the ceiling with different luminous intensity levels was sixteen. And the sum total of photographs of all the combinations of luminous colours and intensity levels considering black also as one of the colours with the luminous ceiling switched off was $[6 \times 16=]$ ninety-six. These photographs were used to program a web-server that projects the 
different lighting scenarios in a virtual environment. Whenever a participant uses the luminous intensity or colour control keys on the prototype interface, the interface sends a signal via wireless Internet to the web-server to gradually fade between the different photographs giving the impression that the participant is actually changing the luminous intensity or colour of the space.

\subsubsection{Test Environment - Virtual}

The interactivated space or test environment for projecting photographs from the web-server was setup in the multimedia room of the School of Architecture and Design at Victoria University of Wellington in New Zealand. The multimedia room consists of a group of five NEC LT 380 projectors with a screen resolution of $1600 \times 1200$ pixels that can be programmed to project a matrix of images on three screens. The screens are at an angle of $120^{\circ}$ with each other and form a part of an incomplete octagon. Participants were seated near the three screens so as to experience an enclosed immersive virtual environment and were asked to use the two prototype interfaces.

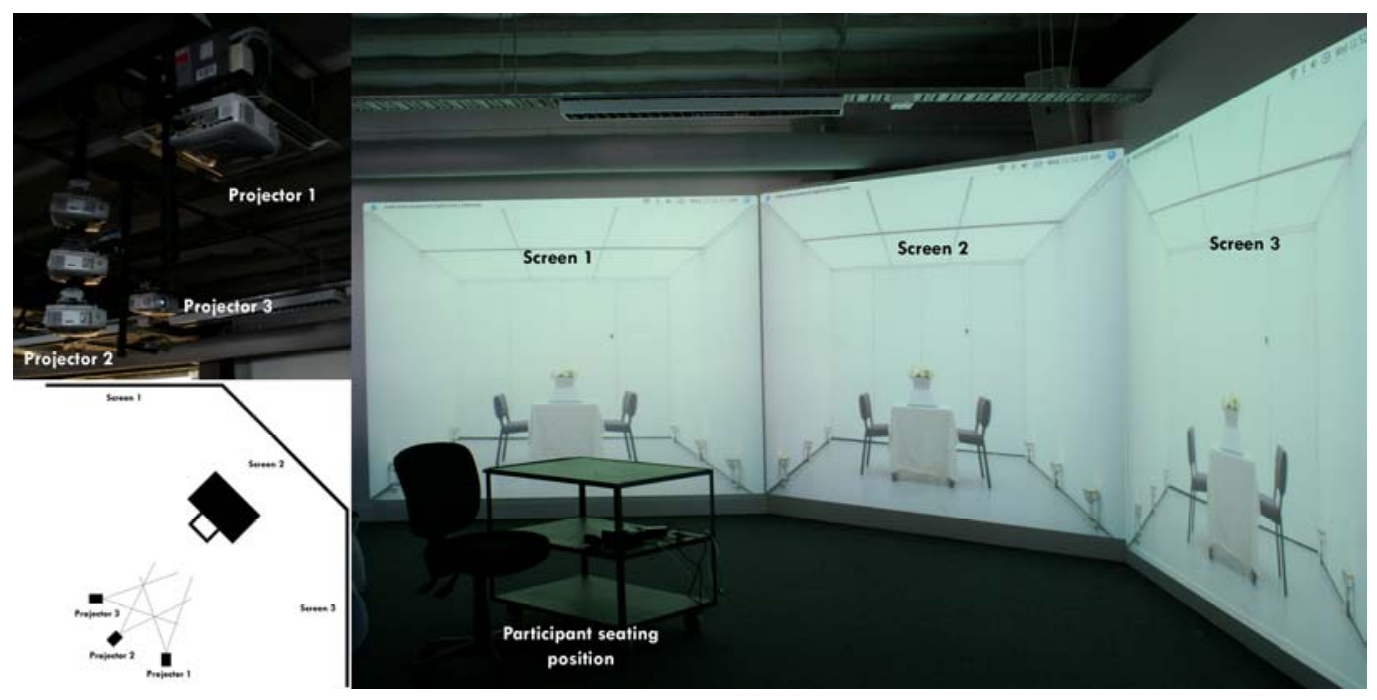

Figure 19 - Test environment [Virtual] 
The questionnaire for the TI study in Appendix 2 first asked participants to study the prototype interface ' $X$ ' and identify visually [eyes only] and then by using the control keys, the control functions of these keys. It then asked participants to select their desired lighting scenario from these three layers: luminous intensity levels with Layers - 1 and - 2 using the dimming control keys, and a luminous colour from Layer - 3 using the colour control keys. It subsequently asked participants to change the luminous intensity levels and colours of these layers, and finally try reverting to the previously selected intensity level and colour. During the entire procedure of performing these control tasks, it asked participants to carefully observe the interface.

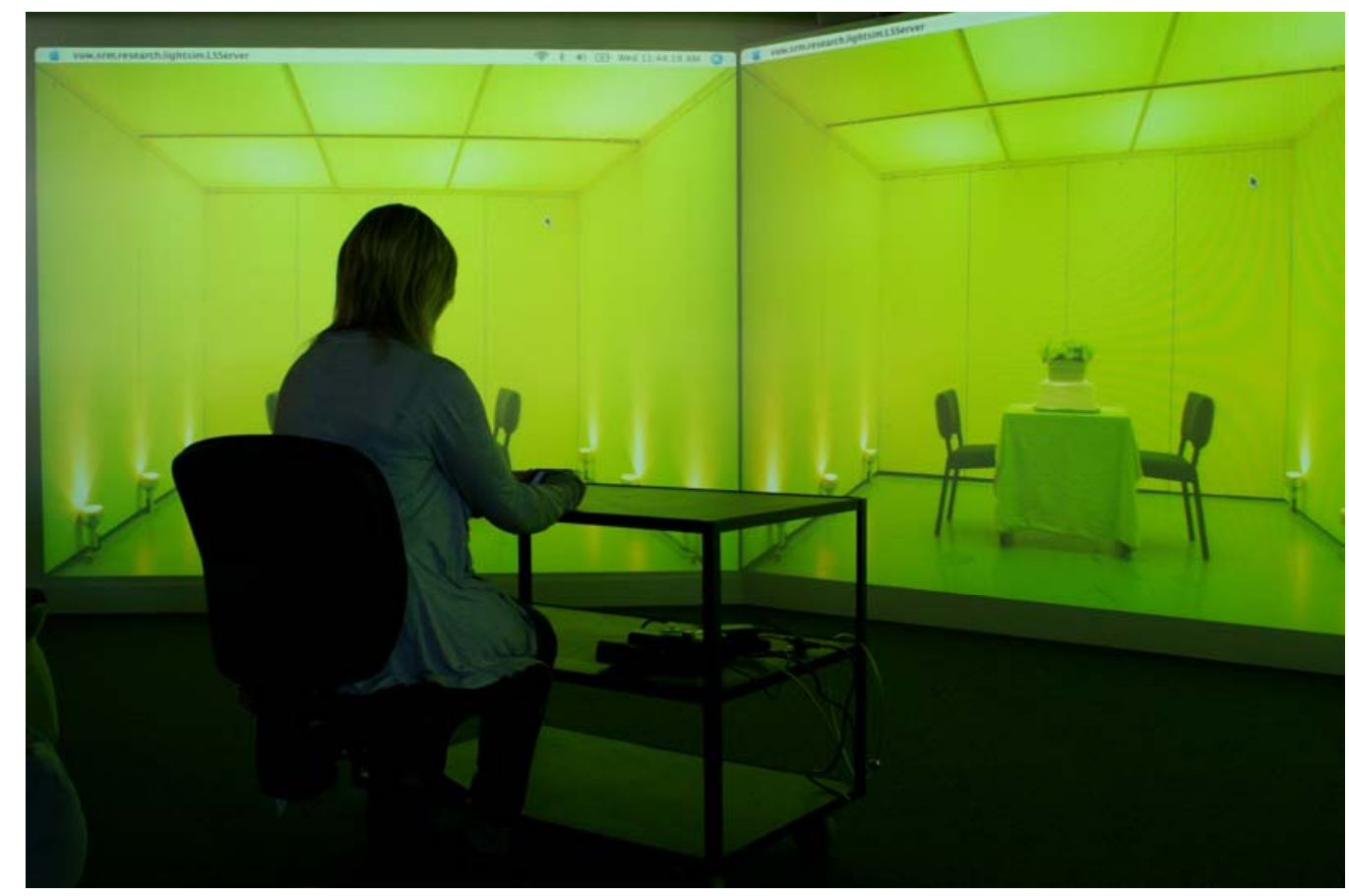

Figure 20 - A participant using the interface in the immersive virtual test environment

The questionnaire also asked participants to use the preset control keys on the interface to save eight of their favourite lighting scenes, and recall their favourite lighting scenes. After having performed the above functions with the prototype interface ' $X$ ', it asked participants to perform 
similar functions with prototype interface ' $Y$.' Once participants were satisfied with use of both the prototype interfaces, the questionnaire in Appendix - 2 asked them to comparatively rate both interfaces in the following manner: the time taken to identify luminous intensity/colour control keys using the 'Learning Speed' scale; the ease of operating the luminous intensity/colour control keys using the 'Ease of Use' scale; the accuracy of being able to repeat selection of luminous intensity/colour using the 'Accuracy' scale; the number of attempts taken to accurately recall their favourite lighting scene; the responsiveness of the interface in providing feedback using the 'Responsiveness' scale. Counterbalancing techniques as mentioned in Section 5.6.2 were used to avoid any form of carryover effects in the repeated measures design of the experiment. For example, the first participant was asked to use the prototype interface ' $X$ ' first and then the prototype interface ' $Y$,' while the second participant was asked to use the prototype interface ' $Y$ ' first and then the prototype interface ' $X$,' and so on.

\subsection{Fabrication: Virtual prototypes}

Dr. Marshall programmed an iPod Touch to be used as a prototype virtual interface to control the fading of the photographs on the webserver from one photograph to another via wireless Internet. A 8GB iPod Touch of physical dimensions $110 \times 61.8 \times 8.5 \mathrm{~mm}$, with a 3.5-inch [diagonal] widescreen Multi-Touch display and 480-by-320-pixel resolution at 163 pixels per inch was selected for the TI study. This was based on participant suggestions from the El study for using a touchscreen based handheld mobile device for controlling the lighting and the principle of contextual experiences for a multi-functional device in context. This prototype virtual interface allows for the programming and 
recall of eight preset scenes. Preece, Rogers, and Sharp argue that prototyping involves producing a limited version of the product with the purpose of answering specific questions about the design's feasibility or appropriateness $(2002$, p. 180). They further argue that prototypes give a better impression of end-user experiences than simple descriptions, and there are different kinds of prototyping that are suitable for different stages of development and for drawing different kinds of information (p. 180).

\subsubsection{Virtual Prototype - Existing interface}

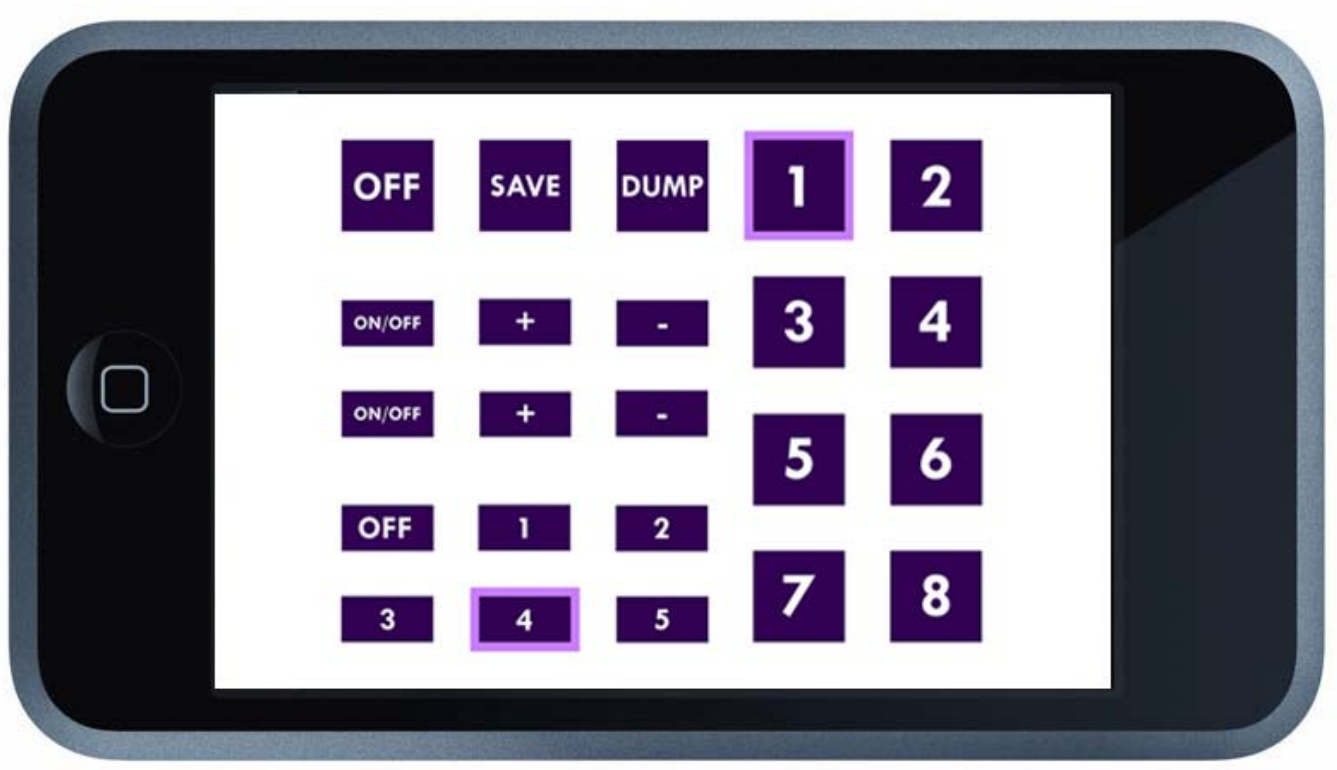

Figure 21 - Virtual Prototype [Existing]

The prototype design for the existing interface was derived after reviewing designs of existing pushbutton and touch interfaces from the manufacturers Dynalite and Helvar (Dynalite, 2008; Helvar, 2007). Three buttons each are allocated for switching and dimming of Layers 1 and - 2: one for switching off the lights and one each for increasing and decreasing their luminous intensity levels. The on/off button is labelled as "ON/OFF" while the increase and decrease buttons are 
labelled as "+" and "-" respectively. Six buttons are allocated for controlling the luminous colours from Layer - 3: an off button for switching off the ceiling lights and one button each for the other five colours from the test environment namely amber, blue, green, red and white. The off button is labelled as "OFF" while the other buttons are labelled using alphanumeric characters " 1 " through " 5 " respectively.

Two buttons are allocated for saving and deleting scenes, and are labelled as "SAVE" and "DUMP" respectively. Eight buttons are allocated for recalling the eight saved preset scenes and labelled " 1 " through " 8 " respectively. Eight scenes can be saved and recalled using these buttons. And finally one button is allocated for switching off all the lights and labelled as "OFF."

\subsubsection{Virtual Prototype - Tangible interface}

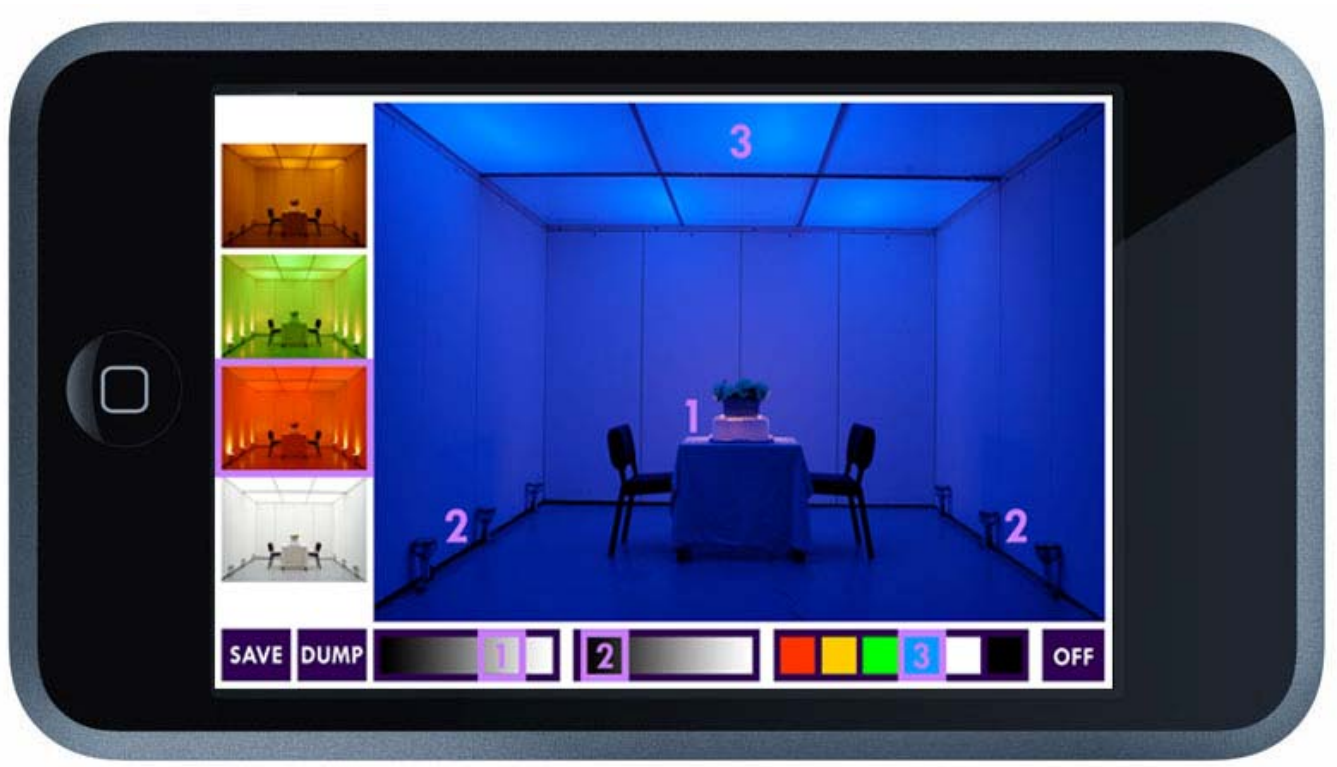

Figure 22 - Virtual Prototype [Tangible]

The prototype design for the tangible interface was derived after reviewing design suggestions provided by participants from the El study, 
along with the principles of controllable representations in Section 4.2.1 and expressive reciprocations in Section 4.3.1. As participants in the El study rated the slider to be accurate and easy to use, two virtual sliders are allocated for controlling the luminous intensity of Layers -1 and -2 . A colour palette based on participants' suggestion from the El study is allocated for controlling Layer - 3: a black off button for switching off the ceiling lights and one button each for the other five colours from the test environment namely amber, blue, green, red and white. Two buttons are allocated for saving and deleting scenes, and are labelled as "SAVE" and "DUMP" respectively. As participants can quickly and accurately recognise iconographic representations of scenes, thumbnails of the saved scenes are allocated on a display scroll-bar when scenes are saved. Eight scenes can be saved, scrolled and recalled using the scroll-bar. The sliders, colour palette, and scroll-bar follow the principle of controllable representations.

A central image of the lit environment with respective alphanumeric cue references of the three lighting layers namely "1," "2," and " 3 " is displayed on the interface to provide a direct and accurate mapping of the layers of lighting and their respective controls. This image is programmed to synchronically and gradually fade in luminous intensity and colours with the actual projected image on the screen when the sliders or colour controllers are used. The central image follows the principle of expressive reciprocations and occupies almost $75 \%$ of the screen space in comparison to the sliders and colour control keys which occupy about $2 \%$. And finally one button is allocated for switching off all the lights and labelled as "OFF." 


\subsection{Execution: TI study}

The TI study was executed within the premises of the Schools of Architecture and Design, Victoria University of Wellington in New Zealand. A brief demographic analysis of the thirty-six participants in Table 15 showed that thirteen are from the age group of 16-24 years, seventeen from the age group of 25-34 years, five from the age group of 35-44 years, and one from the age group of 45-54 years. Eighteen of the participants are male while eighteen are female. Nine of the participants were staff members while the rest twenty-seven were students.

\begin{tabular}{|l|c|}
\hline \multicolumn{1}{|c|}{ Demographics } & Number of participants \\
\hline $16-24$ & 13 \\
\hline $25-34$ & 17 \\
\hline $35-44$ & 5 \\
\hline $45-54$ & 1 \\
\hline \multicolumn{1}{|c|}{ Gender } & 18 \\
\hline Male & 18 \\
\hline Female & \\
\hline University Community & 9 \\
\hline Staff & 27 \\
\hline Students & \\
\hline
\end{tabular}

\subsubsection{Results - Evaluating interfaces}

The test scores for each of the operational definitions obtained from the quantitative data using inferential statistics are listed in Tables 16 and 17. Any other qualitative comments and suggestions made by participants were coded, conceptualised and categorised using the grounded theory coding described in Section 5.5.3 under each of the operational definitions and summarised in Tables 16-17. 


\subsubsection{Evaluating Interfaces - Appearance}

Fifteen out of thirty-six [ $>40 \%$ ] participants commented that the tangible interface looked like a device related to the control of lighting in a room.

\subsubsection{Evaluating Interfaces - Accuracy}

Participants found no difference in the accuracy of the existing and tangible interfaces for controlling luminous intensity. As $p>0.05$, it is accepted that the difference in mean ranks was due to random error. Fourteen $[\approx 39 \%$ ] participants commented that the accuracy of the sliders on the tangible interface could be improved with a numeric graphical scale.

Participants rated the tangible interface as more accurate than the existing interface for selecting their desired luminous colours. The mean ranks for tangible and existing interfaces were 1.64 and 1.36 respectively. These differences in mean ranks are significant as $p<$ 0.05 .

Participants rated the tangible interface as more accurate than the existing interface for recalling their desired preset scenes. Twenty-three out of thirty-six [> $60 \%$ ] participants were able to recall their desired preset scene on the first attempt using the tangible interface while only three out of thirty-six $[<10 \%]$ were able to recall it on the first attempt using the existing interface. 


\subsubsection{Evaluating Interfaces - Learning speed}

Participants took less time to learn about the luminous intensity control function from the tangible interface than the existing interface. The mean ranks for tangible and existing interfaces were 1.83 and 1.17 respectively. These differences in mean ranks have a high significance as $p<0.001$.

Participants took less time to learn about the luminous colour control function from the tangible interface than the existing interface. The mean ranks for tangible and existing interfaces were 1.93 and 1.07 respectively. These differences in mean ranks have a high significance as $p<0.001$.

\subsubsection{Evaluating Interfaces - Responsiveness}

Participants rated the tangible interface as more responsive than the existing interface. The mean ranks for tangible and existing interfaces were 1.68 and 1.32 respectively. These differences in mean ranks are significant as $p<0.05$. Seven $[\approx 19 \%]$ participants even commented that using the tangible interface was more "fun" as it had the "playful" feeling of a "game."

\subsubsection{Evaluating Interfaces - Ease of use}

Participants found no difference in the ease of use of the existing and tangible interfaces for controlling luminous intensity. As $p>0.05$, it is accepted that the difference in mean ranks was due to random error. Eighteen [50\%] participants commented that the sliders on the tangible interface were not very "touch sensitive" which made them difficult to use. Six participants even commented that the luminous intensity control keys on the existing interface were more touch sensitive than the sliders. 
Participants found no difference in the ease of use of the existing and tangible interfaces for controlling luminous colour. As $p>0.05$, it is accepted that the difference in mean ranks was due to random error. Seven participants commented that the colour control keys on the tangible interface were too small for their fingers, which made them difficult to use.

\begin{tabular}{|c|c|c|c|c|c|c|c|}
\hline & & \multicolumn{3}{|c|}{ Existing } & \multicolumn{3}{|c|}{ Tangible } \\
\hline & Dimensions & $M$ & $S D$ & Min-Max & $M$ & $S D$ & Min-Max \\
\hline \multirow{3}{*}{$\begin{array}{l}\text { Luminous } \\
\text { Intensity } \\
\text { Control }\end{array}$} & Accuracy & 2.33 & 0.76 & $1-3$ & 2.17 & 0.77 & $1-3$ \\
\hline & Learning Speed & 2.00 & 0.63 & $1-3$ & 2.86 & 0.42 & $1-3$ \\
\hline & Ease of Use & 3.03 & 0.84 & $1-4$ & 2.75 & 0.87 & $1-4$ \\
\hline \multirow{3}{*}{$\begin{array}{l}\text { Luminous } \\
\text { Colour } \\
\text { Control }\end{array}$} & Accuracy & 2.56 & 0.61 & $1-3$ & 2.81 & 0.52 & $1-3$ \\
\hline & Learning Speed & 2.06 & 0.47 & $1-3$ & 3.00 & 0.00 & $3-3$ \\
\hline & Ease of Use & 3.14 & 0.83 & $1-4$ & 3.56 & 0.77 & $1-4$ \\
\hline $\begin{array}{l}\text { General } \\
\text { Characteristics }\end{array}$ & Responsiveness & 2.03 & 0.81 & $1-3$ & 2.64 & 0.59 & $1-3$ \\
\hline
\end{tabular}

Table 16 - Summary of Means, Standard Deviations and Minimum-Maximum range for the existing and tangible interface prototypes [TI study]

\begin{tabular}{|c|c|c|c|c|c|}
\hline & & Existin & Tangib & & \\
\hline & Dimensions & \multicolumn{2}{|c|}{ Mean Ranks } & $p$ & $\chi^{2}(d f=1)$ \\
\hline \multirow{3}{*}{$\begin{array}{l}\text { Luminous } \\
\text { Intensity } \\
\text { Control }\end{array}$} & Accuracy & 1.56 & 1.44 & 0.450 & 0.571 \\
\hline & Learning Speed & 1.17 & 1.83 & $<0.001$ & 20.571 \\
\hline & Ease of Use & 1.60 & 1.40 & 0.209 & 1.581 \\
\hline Luminous & Accuracy & 1.36 & 1.64 & 0.012 & 6.250 \\
\hline Colour & Learning Speed & 1.07 & 1.93 & $<0.001$ & 31.000 \\
\hline Control & Ease of Use & 1.36 & 1.64 & 0.059 & 3.571 \\
\hline $\begin{array}{l}\text { General } \\
\text { Characteristics }\end{array}$ & Responsiveness & 1.32 & 1.68 & 0.016 & 5.828 \\
\hline
\end{tabular}

Table 17 - Mean ranks for the existing and tangible interface prototypes [TI study]

\subsubsection{Evaluating Interfaces - Summary}

End-user evaluation of the prototype interfaces shows that participants were able to identify the function of a lighting control interface easily 
from the appearance of the tangible interfaces in comparison to the existing interfaces. Identification of the luminous intensity and colour control functions required less time in comparison to the existing interface. They were able to select and repeat a selection of luminous colour much more accurately with the tangible interface in comparison to the existing interface. The visibly perceptible feedback provided by the tangible interface enabled participants to have a conversational type of interaction in comparison to the existing interface. However, they did not find any difference in the accuracy of selecting luminous intensities from these interfaces. Similarly, they did not find any difference in the ease of using these interfaces.

\subsubsection{Results - Designing interfaces}

\begin{tabular}{|c|c|c|c|}
\hline & \multirow[t]{2}{*}{ Participants' descriptive text/drawings } & \multicolumn{2}{|c|}{ No: of Participants } \\
\hline & & Existing & Tangible \\
\hline \multirow{4}{*}{ Comments } & Interface looks like a device for controlling lighting & 0 & 15 \\
\hline & $\begin{array}{l}\text { Interface enables accurate recall of saved lighting } \\
\text { scenes in the first attempt }\end{array}$ & 3 & 23 \\
\hline & Interface more fun and pleasurable to use & 0 & 7 \\
\hline & Prefer the overall design of the interface & 0 & 33 \\
\hline \multirow{3}{*}{ Suggestions } & $\begin{array}{l}\text { Improve the accuracy of the sliders with a numeric } \\
\text { graphical scale }\end{array}$ & NA & 14 \\
\hline & $\begin{array}{l}\text { Combine features of conventional and tangible } \\
\text { interface }\end{array}$ & 4 & 4 \\
\hline & $\begin{array}{l}\text { Overlay control keys on the images of the lighting } \\
\text { layers to give a feeling of direct interaction with the } \\
\text { lighting }\end{array}$ & NA & 2 \\
\hline
\end{tabular}

Table 18 - Summary of participant comments and suggestions [TI study]

The qualitative data in the form of design suggestions made by participants are coded, conceptualised and categorised using the grounded theory coding described in Section 5.5.3 under the category for designing future tangible interfaces. The system used is to list all suggestions made by participants in terms of continuous prose and listed 
answers. These are then quantified by grouping all similar descriptions under the number of participants who made these suggestions in Table 18.

\subsection{Interpretation: TI study}

The results of the TI study were analysed to test hypotheses about future tangible interfaces. The differences in percentages of favourable responses or mean ranks between the stages as well as their significance levels were used as a means of comparing the quantitative test scores. The qualitative comments were further used to verify the hypotheses. The interpretation of the $\mathrm{TI}$ study also enables assessment of the overall hypotheses of this thesis research.

\subsubsection{Analysis - Testing hypotheses}

The test scores provide quantitative evidence to test hypotheses, while participant comments provide qualitative evidence to help understand participant responses.

\subsubsection{Hypothesis \# 2}

- Expressive iconographic and typographic representations of luminous intensities/colours enable quicker recognition and more accurate selection of the desired luminous intensities/colours than no representations.

The test scores only provide adequate evidence to show that expressive iconographic representations of luminous intensities enable quicker recognition of the luminous intensity control function than no representations. The tangible interface with expressive iconographic representations of luminous intensities scored a higher mean rank than the 
existing interface for learning speed at a high significance level. However, participants do not find any difference in the accuracy of these interfaces. From the participants' comments, the sliders on the tangible interface could be made more effective by improving their touch sensitivity and providing a numeric graphical scale. Therefore this hypothesis is partially supported.

The test scores provide adequate evidence to show that expressive iconographic representations of luminous colours enable quicker recognition and more accurate selection of the desired luminous colours than no representations. The tangible interface with expressive iconographic representations of luminous colours scored a higher mean rank than the existing interface for learning speed and accuracy, although at different significance levels. However, from the participants' comments, the colour palette on the tangible interface could be made more effective by increasing the size of the colour control keys. Therefore this hypothesis is adequately supported.

\subsubsection{Hypothesis \# 5}

- Interfaces that provide noticeable visual changes during manipulation are considered more responsive than interfaces that do not provide these changes.

The test scores and participant comments provide adequate evidence to show that interfaces that provide noticeable visual changes during manipulation are considered more responsive than interfaces that do not provide these changes. The tangible interface with a visually responsive touch-screen scored a higher mean rank than the existing interface for responsiveness, although at a low significance level. From the participants' comments, the sliders on the tangible interface could be 
made more responsive by improving their touch sensitivity. Therefore this hypothesis is adequately supported.

\subsubsection{Hypothesis \# 6}

- Interfaces that represent and map the lit environment in a manner that gives the impression of direct interaction with, and control of, the available lighting layers enable quicker recognition and more effective operation of the luminous intensity/colour and preset control functions than interfaces that do not map these layers.

The test scores only provide adequate evidence to show that physical designs of interfaces that represent and map the lit environment in a manner that gives the impression of direct interaction with, and control of, the available lighting layers enable quicker recognition of the luminous intensity control function than interfaces that do not map these layers. The tangible interface with directly mapped representations of the lighting layers and control of the luminous intensity scored a higher mean rank than the existing interface for learning speed at a high significance level. However, participants do not find any difference in the accuracy of these interfaces. From the participants' comments, the sliders on the tangible interface could be made more effective by improving their touch sensitivity and providing a numeric graphical scale. Therefore this hypothesis is partially supported.

The test scores provide adequate evidence to show that interface designs that represent and map the lit environment in a manner that gives the impression of direct interaction with, and control of, the available lighting layers enable quicker recognition and more effective operation of the luminous colour control function than interfaces that do not map these layers. The tangible interface with directly mapped representations of the lighting layers and control of the luminous colour scored a higher 
mean rank than the existing interface for learning speed and accuracy, although at different significance levels. However from the participants' comments, the colour palette on the tangible interface could be made more effective by increasing the size of the colour control keys. Therefore this hypothesis is adequately supported.

\subsubsection{Hypothesis \# 7}

- Interfaces that represent and map the lit environment in a manner that gives the impression of direct interaction with, and control of, the available lighting layers are more acceptable than interfaces that do not map these layers.

Participant comments and design suggestions provide adequate evidence to show that interfaces that represent and map the lit environment in a manner that gives the impression of direct interaction with, and control of, the available lighting layers are more acceptable than interfaces that do not map these layers. Thirty-three out of thirty-six [ $>90 \%]$ participants commented that they prefer the overall design of the tangible interface to the existing interface. Two participants even expressed their desire for direct interaction with the lighting. Therefore this hypothesis is adequately supported.

\subsection{Chapter Inference}

Chapter 7 has described the implemented procedure and results of the $\mathrm{TI}$ study. The study was conducted with participants from different departments of a Victoria University of Wellington in New Zealand. A real test environment with three layers of lighting was setup and photographed with different light settings. These photographs were then used to create an immersive virtual test environment by projecting them 
on to three screens using a web-server. An iPod Touch was programmed to display two different virtual prototypes of lighting control interfaces namely existing and tangible for controlling this environment via wireless Internet.

The analysis of the quantitative and qualitative data obtained from the $\mathrm{TI}$ study shows that existing designs of preset control interfaces either convey partial or no information at all about the different lighting scenarios they are intended to create and how end-users can use them. Quicker recognition and more effective operation is facilitated by interface designs that represent and map the lit environment in a manner that gives the impression of direct interaction with, and control of, the available lighting layers for controlling luminous intensity/colour. However, the touch sensitivity of the sliders on the tangible interface needs improvement. Similarly, information that is richly represented in the form of expressive iconography and typography facilitates quicker recognition and accurate selection of the luminous intensities/colours and preset scenes. Existing interfaces for preset control use alphanumeric characters for representing luminous intensity/colours and preset lighting scenes for recall, which do not convey any information about the functions. Interactive property of interfaces can also be experienced when the interface provides rich visual responses with noticeable visual changes upon manipulation. Existing interfaces for preset control do not provide the rich visual responses required for end-users to experience its interactive property during explorative manipulation.

In Chapter 7, implementation of the $\mathrm{TI}$ study for observing and documenting end-user behaviour with a tangible lighting control interface has been described. The documented results on end-user evaluation of these interfaces are used to test hypotheses about future tangible 
interfaces. This project will now have to be reviewed to assess its progress and arrive at any new findings. Chapter 8 will review the project, while Chapter 9 will discuss future directions for research and development based upon the findings of this thesis research. 


\section{CHAPTER EIGHT}

\section{Reviewing 'Tangible Lighting Controls'}

\subsection{Chapter Intent}

Chapter 8 reviews this $\mathrm{PhD}$ project. The principal aim of this $\mathrm{PhD}$ project of establishing the concept of tangibility in lighting control systems has been achieved. The surveyed end-users were able to better understand the lighting control functions as well as derive pleasure in using the prototype tangible lighting control interface. The overall aims of promoting end-user interaction with lit environments through tangible lighting control interfaces and understanding the real needs of end-users have also been achieved. The project requires reviewing to assess whether its conclusions support the overall research hypotheses. The assessment will also include any further conclusions that have been arrived at during the course of this thesis research.

Section 8.2 will make an overall assessment of the project conclusions supporting the research hypotheses, before Section 8.3 lists a whole new set of conclusions.

\subsection{Research Hypotheses Related Conclusions}

Chapter 3 described the hypothetical nature of interface designs sought by end-users for improved usability and end-user experience based on 
the literature reviews done in Chapters 1 and 2. Chapter 1 described how architectural lighting designers could design quality lighting environments using tangible interaction. Chapter 2 further described how personalised interactive user-controlled electric lighting systems can provide end-users with the freedom to select desired lighting scenarios, thereby providing rich sensory experiences.

This thesis research then focussed on the physical interaction possibilities of tangible lighting control interfaces to improve usability and end-user experience by deriving design principles and key characteristics in Chapter 4. It also focussed on understanding end-user understanding of usability and end-user experience of lighting control interfaces by designing two interactive studies in Chapter 5: Existing interface [El] and Tangible interface [TI] study. Based on the results of the El study in Chapter 6, it has demonstrated that existing designs of lighting control interfaces can be improved to meet end-user requirements for usability and end-user experience with tangible interaction.

Finally, based on the results of TI study in Chapter 7, it has demonstrated that end-user interaction with lit environments can be promoted through tangible lighting control interfaces. In doing so, the project has established that the two aspects of interactive lighting control can be integrated using tangible interaction: ease of understanding control functions and pleasure of performing control tasks. 


\subsection{Further Conclusions}

During the course of this project, six further conclusions were drawn based on the literature reviews and results of the interactive studies. The following paragraphs describe these conclusions.

\subsubsection{Conclusion \# 1}

Measures beyond 'ease of use' have to be used for evaluating the usability and end-user experience of lighting control interfaces. The results of the Tangible interface study in Chapter 7 show that end-users prefer using tangible lighting control interfaces even if they are not very easy to use. For example, despite the fact that participants did not find any difference in the ease of using the two prototype interfaces, almost all participants prefer the overall design of the prototype tangible interface. Overbeeke, Diajadiningrat, Hummels, and Wensveen argue that there is more to usability than ease of use, as bringing together "contexts of experience" and "aesthetics of interaction" is not for making a function as easy to access as possible, but for making the unlocking of the functionality contribute to the overall experience $(2002$, p. 11). For example, end-users may choose to work with the lighting control interface despite it being difficult to use because it is challenging, seductive, playful, surprising, memorable or rewarding, resulting in enjoyment of the experience. Hornecker and Bruns in their study of the "Sensoric Garden" installations that combine "multimedia with novel multi-modal interaction techniques" reinforce this by reporting that this kind of event cannot be evaluated in terms of usability, as usability only ensures that systems are effective to use and do not frustrate end-users, whereas "joy of use" refers to positive enjoyment and can motivate end-users to use a product regardless of traditional measures of usability $(2004$, p. 3$)$. 


\subsubsection{Conclusion \# 2}

Values beyond system functionality have to be taken into consideration while designing and marketing lighting control interfaces. The results of the Existing interface study in Chapter 6 show that end-user requirements differ significantly from the current specifications put forward by manufacturers and distributors. For example, although the pushbutton dimming interface enables accurate selection of luminous intensity levels, participants have a preference for the rotary dimmer due to their interactive qualities. As can be seen in Table 19, some participants even suggested that although existing pushbutton and slide interfaces have two separate actions of switching and dimming, they would prefer one streamlined action for both functions.

\begin{tabular}{|c|c|c|}
\hline Interface type & Illustration & $\begin{array}{l}\text { End-user suggestions for better existing } \\
\text { interfaces }\end{array}$ \\
\hline $\begin{array}{l}\text { Type: Pushbutton } \\
\text { Manufacturer: Lutron } \\
\text { Model: Rania } \\
\text { Source: } \\
\text { http://www.lutron.com }\end{array}$ & 1 & $\begin{array}{l}\text { - One streamlined action for switching and } \\
\text { dimming } \\
\text { - Control handle with surface texture or grip }\end{array}$ \\
\hline $\begin{array}{l}\text { Type: Rotary dimmer } \\
\text { Manufacturer: Anchor } \\
\text { Model: Rotary }\end{array}$ & (2) & $\begin{array}{l}\text { - Iconic or numeric depiction in the form of a } \\
\text { graphical scale to improve accuracy } \\
\text { - Control handle with surface texture or grip } \\
\text { - Control handle with larger surface area }\end{array}$ \\
\hline $\begin{array}{l}\text { Type: Slide dimmer } \\
\text { Manufacturer: Lutron } \\
\text { Model: Lyneo } \\
\text { Source: } \\
\text { http://www.lutron.com }\end{array}$ & $\begin{array}{l}1 \\
1 \\
\end{array}$ & $\begin{array}{l}\text { - Iconic or numeric depiction in the form of a } \\
\text { graphical scale to improve accuracy } \\
\text { - One streamlined action for switching and } \\
\text { dimming } \\
\text { - Control handle with surface texture or grip } \\
\text { - Control handle with larger surface area }\end{array}$ \\
\hline
\end{tabular}

Table 19 - Summary of participant suggestions for better existing interfaces from Existing interface study 


\subsubsection{Conclusion \# 3}

Listening and acknowledging end-users' experience and understanding of lighting control interface leads to interface designs that work well for end-users. The results of the interactive studies in Chapters 6 and 7 show that end-users prefer using interfaces that have incorporated their own ideas. For example, the incorporated design suggestions made by participants of the Existing interface study in Chapter 6, led to a wide acceptance of the prototype tangible interface design by participants of the Tangible interface study in Chapter 7. Some participants from the Tangible interface study even suggested that by incorporating certain properties of existing interfaces, the prototype tangible interface design could be further refined.

\subsubsection{Conclusion \# 4}

Physical actions beyond the "monotonous string of motorically trivial actions" of pushing, rotating and sliding as described by Buur, Jensen, and Diajadiningrat have to be explored while designing lighting control interfaces (2004, p. 186). The results of the interactive studies from Chapters 6 and 7 show that end-users prefer using lighting control interfaces that offer expression of physical actions that match their cognitive, perceptual-motor, and emotional skills. For example, most participants of the Existing interface study in Chapter 6 were not able to identify or relate with the custom fabricated pushbutton, rotary and slide interfaces used for luminous colour control. One participant even suggested that lighting control interfaces could sense physical actions such as pressure provided by the hand to dim the lighting. 


\subsubsection{Conclusion \# 5}

Multi-faceted interfaces work well for end-users. The results of the Existing interface study in Chapter 6 show that end-users prefer one interface to perform several different functions. For example, some participants suggested that one interface could perform all the functions of switching, dimming and preset control. One participant even suggested that interfaces could resemble and perform function of handheld objects in everyday use.

\subsubsection{Conclusion \# 6}

The realm of lighting controls has not been explored to its full potential in lighting research. Literature reviews of existing studies in lighting controls show that factors such as usability and end-user experience of lighting control systems have not yet been explored in detail. Additionally, detailed field studies on end-user evaluation of lighting control systems in interior environments other than office environments have not been carried out. 


\section{CHAPTER NINE}

\section{Discussing Future Trends}

\subsection{Directions for Future Work}

While the principal aim of the thesis research has been achieved in that a prototype tangible lighting control interface has been designed and tested that promotes end-user interaction with lit environments, there are several other related areas that have a potential for exploration. Further investigation should be undertaken in the following areas:

- The prototype interfaces used in the test only provided a limited range of luminous intensities/colour and lighting scenes. Subsequent prototypes should provide a larger range of luminous intensities/colours and lighting scenes thereby challenging end-user skills.

- The prototype interfaces used in the test only provided iconographic representations. Subsequent prototypes should provide options of typing typographic texts to support the iconographic representations.

- Given the fact that the entire testing procedure of the prototype interfaces was a mock-up in a virtual environment, this interface should be tested for programming and setting different lighting scenarios in a real-life proof-of-concept demonstration. An obvious next step will be to enable user-evaluation of a practical demonstration of the interface as a post-doctoral research project. 
- The post-doctoral project can also take into consideration the influence of age and gender on the individual preferences of lighting control interfaces by using larger sample sizes.

- The control actions for selecting luminous intensities and colours should explore actions beyond the 2D actions of touching and sliding so that end-users' cognitive, perceptual-motor and emotional skills are challenged. For example, Ann can select the hue, saturation and brightness of the lighting in her bedroom by first 'touching' the right hue with her index finger, then use the rest of her fingers to determine the 'size' of the saturation, and finally 'elongate' the brightness mound with her fingers up/down in 3D to select the right brightness.

- Tangible interaction concepts such as embodied interaction with the lighting should be further explored where the lighting in the room adjusts itself according to the number of people, their respective physical position in the space, and their moods determined by the intensity of their physical activities. For example, Bruce can have a few guests over in his living room for a dance party today, read a nice book lying on the couch tomorrow, and have a card night the day after, while the lighting quietly adjusts itself according to the people and their physical activity so it is just right!

- The tangible lighting control interfaces that have been discussed so far in this PhD project are designed to operate with DALI or DMX control systems that cater only to a market segment involving corporate bodies or high-end residences due to the high installation costs involved. However, with the new breed of economically viable LED lighting technologies and touch-screen mobile devices, a home PC-based lighting control system should be designed to make the tangible interaction concept available to the masses. 


\section{APPENDIX 1}

\section{Questionnaire - Existing interface study}




\section{EXPLORATION INTO LIGHTING CONTROL DEVICES}

Before you start-off with the experiments please help yourself with some drinks and snacks!

\section{GENERAL INFORMATION}
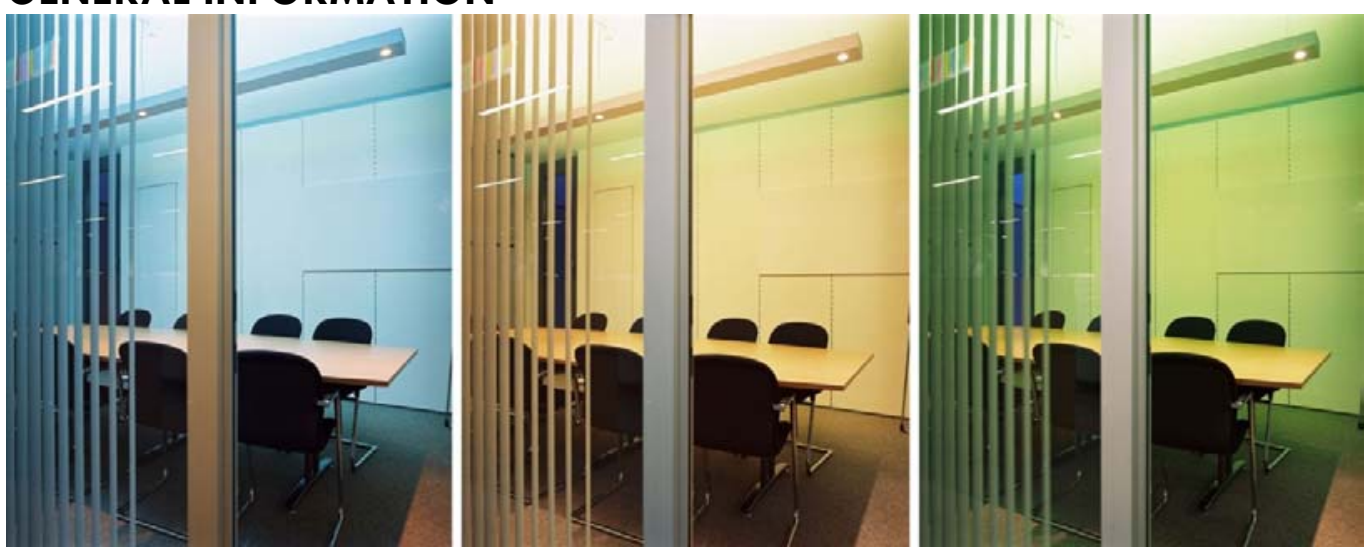

Take a careful look at the image above (Photo courtesy: L-Plan Lighting Design/Hermes Photography). Imagine yourself in this space where you can actually interact with the lighting. The space gives you the option of changing the brightness and colours of light or select lighting scenes (e.g. dinner or meeting scene). Devices used for controlling and selecting lighting are called interfaces. You can change brightness with a dimmer, change colours with a colour controller or select scenes with a scene selector. This survey is about these devices.

\section{PREVIOUS EXPERIENCE WITH LIGHTING CONTROLS}

\begin{tabular}{|l|l|l|}
\hline \multicolumn{3}{|c|}{ Have you ever used devices to: } \\
\hline Turn on or off light sources? & Yes & No \\
\hline Increase or decrease brightness of light sources? & Yes & No \\
\hline Change colours of light sources? & Yes & No \\
\hline Select different lighting scenes? & Yes & No \\
\hline
\end{tabular}

\section{QUESTIONNAIRE ANSWERING TIPS}

On a scale of $i$-iv, where "iv" means very easy to use and " $i$ " means very difficult to use, the ratings of three devices $X, Y$ and $Z$ can be:

\begin{tabular}{|c|c|c|c|c|}
\hline $\begin{array}{c}\text { Interface } \\
\text { Type }\end{array}$ & $\begin{array}{c}\text { Very } \\
\text { difficult to } \\
\text { use }\end{array}$ & $\begin{array}{c}\text { Difficult to } \\
\text { use }\end{array}$ & Easy to use & $\begin{array}{c}\text { Very easy to } \\
\text { use }\end{array}$ \\
\hline $\mathbf{X}$ & $\mathrm{i}$ & ii & iii & iv \\
\hline $\mathbf{Y}$ & $\mathrm{i}$ & $\mathrm{ii}$ & $\mathrm{iii}$ & iv \\
\hline $\mathbf{Z}$ & $\mathrm{i}$ & $\mathrm{ii}$ & iii & iv \\
\hline
\end{tabular}




\section{EXPERIMENT - 1}

The first experiment is about three devices namely $\mathbf{X}, \mathbf{Y} \& \mathbf{Z}$. Carefully study them.

\section{FIRST IMPRESSION}

- Which of these dimming devices impresses you the most?

\begin{tabular}{|c|c|c|c|c|}
\hline $\begin{array}{c}\text { Interface } \\
\text { Type }\end{array}$ & $\begin{array}{c}\text { Not } \\
\text { impressed } \\
\text { at all }\end{array}$ & $\begin{array}{c}\text { Not } \\
\text { impressed }\end{array}$ & Impressed & $\begin{array}{c}\text { Very } \\
\text { impressed }\end{array}$ \\
\hline $\mathbf{X}$ & $\mathrm{i}$ & $\mathrm{ii}$ & $\mathrm{iii}$ & $\mathrm{iv}$ \\
\hline $\mathbf{Y}$ & $\mathrm{i}$ & $\mathrm{ii}$ & $\mathrm{iii}$ & $\mathrm{iv}$ \\
\hline $\mathbf{Z}$ & $\mathrm{i}$ & $\mathrm{ii}$ & $\mathrm{iii}$ & $\mathrm{iv}$ \\
\hline
\end{tabular}

\section{APPEARANCE}

- After closely studying $X, Y \& Z$, think of how these devices will be used.

- Which of these looks like a dimmer for increasing or decreasing brightness?

\begin{tabular}{|c|c|c|c|}
\hline \multirow{3}{*}{ 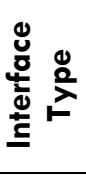 } & $X$ & $\begin{array}{l}\text { Looks like } \\
\text { a dimmer }\end{array}$ & $\begin{array}{c}\text { Does not look like } \\
\text { a dimmer }\end{array}$ \\
\hline & $\mathbf{Y}$ & $\begin{array}{l}\text { Looks like a } \\
\text { a dimmer }\end{array}$ & $\begin{array}{c}\text { Does not look like } \\
\text { a dimmer }\end{array}$ \\
\hline & $\mathbf{Z}$ & $\begin{array}{l}\text { Looks like a } \\
\text { a dimmer }\end{array}$ & $\begin{array}{c}\text { Does not look like } \\
\text { a dimmer }\end{array}$ \\
\hline
\end{tabular}

- Which of these looks like a colour controller for changing light colours?

\begin{tabular}{|c|c|c|c|}
\hline \multirow{3}{*}{ 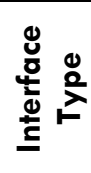 } & $X$ & $\begin{array}{c}\text { Looks like } \\
\text { a colour controller }\end{array}$ & $\begin{array}{l}\text { Does not look like } \\
\text { a colour controller }\end{array}$ \\
\hline & $\mathbf{Y}$ & $\begin{array}{c}\text { Looks like } \\
\text { a colour controller }\end{array}$ & $\begin{array}{l}\text { Does not look like } \\
\text { a colour controller }\end{array}$ \\
\hline & $\mathbf{Z}$ & $\begin{array}{c}\text { Looks like } \\
\text { a colour controller }\end{array}$ & $\begin{array}{l}\text { Does not look like } \\
\text { a colour controller }\end{array}$ \\
\hline
\end{tabular}

\section{GRABBABILITY}

- Grab, feel and move the movable parts of the dimmers $X, Y$ \& $Z$ individually.

- See how grabbable the movable parts are in your hands.

- Now rate each one of them according to their grabbability.

\begin{tabular}{|c|c|c|c|}
\hline \multirow{3}{*}{ 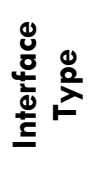 } & $X$ & $\begin{array}{l}\text { Easy } \\
\text { to grab }\end{array}$ & $\begin{array}{l}\text { Hard } \\
\text { to grab }\end{array}$ \\
\hline & $\mathbf{Y}$ & $\begin{array}{c}\text { Easy } \\
\text { to grab }\end{array}$ & $\begin{array}{l}\text { Hard } \\
\text { to grab }\end{array}$ \\
\hline & $\mathbf{Z}$ & $\begin{array}{l}\text { Easy } \\
\text { to grab }\end{array}$ & $\begin{array}{l}\text { Hard } \\
\text { to grab }\end{array}$ \\
\hline
\end{tabular}

\section{PLEASE GIVE REASONS FOR YOUR ANSWERS}




\section{EXPERIMENT - 2}

The second experiment is to use $\mathbf{X}, \mathbf{Y} \& \mathbf{Z}$ as dimmers for increasing and decreasing the brightness of light.

\section{EASE OF USE}

- Use the dimmers $X, Y \& Z$ individually to change brightness a number of times.

- Now rate each one of them according to the ease of use.

\begin{tabular}{|c|c|c|c|c|}
\hline $\begin{array}{c}\text { Interface } \\
\text { Type }\end{array}$ & $\begin{array}{c}\text { Very } \\
\text { difficult to } \\
\text { use }\end{array}$ & $\begin{array}{c}\text { Difficult to } \\
\text { use }\end{array}$ & Easy to use & $\begin{array}{c}\text { Very easy to } \\
\text { use }\end{array}$ \\
\hline $\mathbf{X}$ & $\mathrm{i}$ & $\mathrm{ii}$ & $\mathrm{iii}$ & iv \\
\hline $\mathbf{Y}$ & $\mathrm{i}$ & $\mathrm{ii}$ & $\mathrm{iii}$ & iv \\
\hline $\mathbf{Z}$ & $\mathrm{i}$ & $\mathrm{ii}$ & $\mathrm{iii}$ & iv \\
\hline
\end{tabular}

\section{ACCURACY OF SELECTING PREVIOUS BRIGHTNESS}

- Use the dimmers $X, Y \& Z$ individually to first select a brightness level (of say $50 \%)$.

- Then select the minimum brightness level.

- Again try selecting the previous brightness level (50\%).

- Now rate each one of them according to the accuracy with which you can select the previous brightness level.

\begin{tabular}{|c|c|c|c|}
\hline $\begin{array}{c}\text { Interface } \\
\text { Type }\end{array}$ & Inaccurate & $\begin{array}{c}\text { Sometimes } \\
\text { accurate, } \\
\text { sometimes } \\
\text { not }\end{array}$ & Accurate \\
\hline $\mathbf{X}$ & $\mathrm{i}$ & $\mathrm{ii}$ & $\mathrm{iii}$ \\
\hline $\mathbf{Y}$ & $\mathrm{i}$ & $\mathrm{ii}$ & $\mathrm{iii}$ \\
\hline $\mathbf{Z}$ & $\mathrm{i}$ & $\mathrm{ii}$ & $\mathrm{iii}$ \\
\hline
\end{tabular}

\section{TOUCH RESPONSE}

- Use the dimmers $X, Y$ \& $Z$ individually to gradually increase or decrease brightness.

- Observe the gradual change in brightness.

- Now rate each one of them according to the response you get in brightness change while gradually using them.

\begin{tabular}{|c|c|c|c|}
\hline $\begin{array}{c}\text { Interface } \\
\text { Type }\end{array}$ & $\begin{array}{c}\text { Not } \\
\text { responsive }\end{array}$ & $\begin{array}{c}\text { Somewhat } \\
\text { responsive }\end{array}$ & $\begin{array}{c}\text { Very } \\
\text { responsive }\end{array}$ \\
\hline $\mathbf{X}$ & $\mathrm{i}$ & $\mathrm{ii}$ & $\mathrm{iii}$ \\
\hline $\mathbf{Y}$ & $\mathrm{i}$ & $\mathrm{ii}$ & $\mathrm{iii}$ \\
\hline $\mathbf{Z}$ & $\mathrm{i}$ & $\mathrm{ii}$ & $\mathrm{iii}$ \\
\hline
\end{tabular}

\section{COMMENTS}




\section{EXPERIMENT - 3}

The third experiment is to use $X, Y \& Z$ as colour controllers for changing the light colours.

\section{EASE OF USE}

- Use the colour controllers $X, Y \& Z$ individually to change light colours a number of times.

- Now rate each one of them according to the ease of use.

\begin{tabular}{|c|c|c|c|c|}
\hline $\begin{array}{c}\text { Interface } \\
\text { Type }\end{array}$ & $\begin{array}{c}\text { Very } \\
\text { difficult to } \\
\text { use }\end{array}$ & $\begin{array}{c}\text { Difficult to } \\
\text { use }\end{array}$ & Easy to use & $\begin{array}{c}\text { Very easy to } \\
\text { use }\end{array}$ \\
\hline $\mathbf{X}$ & $\mathrm{i}$ & $\mathrm{ii}$ & $\mathrm{iii}$ & $\mathrm{iv}$ \\
\hline $\mathbf{Y}$ & $\mathrm{i}$ & $\mathrm{ii}$ & $\mathrm{iii}$ & $\mathrm{iv}$ \\
\hline $\mathbf{Z}$ & $\mathrm{i}$ & $\mathrm{ii}$ & $\mathrm{iii}$ & $\mathrm{iv}$ \\
\hline
\end{tabular}

\section{ACCURACY OF SELECTING PREVIOUS COLOUR}

- Use the colour controllers $X, Y \&$ \& individually to first select a light colour.

- Then select a different light colour.

- Again try selecting the previous light colour.

- Now rate each one of them according to the accuracy with which you can select the previous light colour.

\begin{tabular}{|c|c|c|c|}
\hline $\begin{array}{c}\text { Interface } \\
\text { Type }\end{array}$ & Inaccurate & $\begin{array}{c}\text { Sometimes } \\
\text { accurate, } \\
\text { sometimes } \\
\text { not }\end{array}$ & Accurate \\
\hline $\mathbf{X}$ & $\mathrm{i}$ & $\mathrm{ii}$ & $\mathrm{iii}$ \\
\hline $\mathbf{Y}$ & $\mathrm{i}$ & $\mathrm{ii}$ & $\mathrm{iii}$ \\
\hline $\mathbf{Z}$ & $\mathrm{i}$ & $\mathrm{ii}$ & $\mathrm{iii}$ \\
\hline
\end{tabular}

\section{TOUCH RESPONSE}

- Use the colour controllers $X, Y$ \& $Z$ individually to gradually change light colours.

- Observe the gradual change in light colours.

- Now rate each one of them according to the response you get in colour change while gradually using them.

\begin{tabular}{|c|c|c|c|}
\hline $\begin{array}{c}\text { Interface } \\
\text { Type }\end{array}$ & $\begin{array}{c}\text { Not } \\
\text { responsive } \\
\text { at all }\end{array}$ & $\begin{array}{c}\text { Somewhat } \\
\text { responsive }\end{array}$ & $\begin{array}{c}\text { Very } \\
\text { responsive }\end{array}$ \\
\hline $\mathbf{X}$ & $\mathrm{i}$ & $\mathrm{ii}$ & $\mathrm{iii}$ \\
\hline $\mathbf{Y}$ & $\mathrm{i}$ & $\mathrm{ii}$ & $\mathrm{iii}$ \\
\hline $\mathbf{Z}$ & $\mathrm{i}$ & $\mathrm{ii}$ & $\mathrm{iii}$ \\
\hline
\end{tabular}

\section{COMMENTS}




\section{EXPERIMENT - 4}

The fourth experiment is about selecting lighting scenes using devices called scene selectors. Carefully study the scene selectors A, B, C, D \& E and the four different scenes that can be selected with them.

\section{FIRST IMPRESSION}

- Which of the scene selectors impresses you the most?

\begin{tabular}{|c|c|c|c|c|}
\hline $\begin{array}{c}\text { Interface } \\
\text { Type }\end{array}$ & $\begin{array}{c}\text { Not } \\
\text { impressed } \\
\text { at all }\end{array}$ & $\begin{array}{c}\text { Not } \\
\text { impressed }\end{array}$ & Impressed & $\begin{array}{c}\text { Very } \\
\text { impressed }\end{array}$ \\
\hline A & i & ii & iii & iv \\
\hline B & i & ii & iii & iv \\
\hline C & i & ii & iii & iv \\
\hline D & i & ii & iii & iv \\
\hline E & i & ii & iii & iv \\
\hline
\end{tabular}

\section{SCENE ACCURACY}

- Use the scene selectors A, B, C, D \& E to select the different lighting scenes.

- Now rate each one of the selectors in terms of how accurately they describe the different lighting scenes.

\begin{tabular}{|c|c|c|c|}
\hline $\begin{array}{c}\text { Interface } \\
\text { Type }\end{array}$ & Inaccurate & $\begin{array}{c}\text { Sometimes } \\
\text { accurate, } \\
\text { sometimes } \\
\text { not }\end{array}$ & Accurate \\
\hline A & $\mathrm{i}$ & $\mathrm{ii}$ & $\mathrm{iii}$ \\
\hline B & $\mathrm{i}$ & $\mathrm{ii}$ & $\mathrm{iii}$ \\
\hline C & $\mathrm{i}$ & $\mathrm{ii}$ & $\mathrm{iii}$ \\
\hline D & $\mathrm{i}$ & $\mathrm{ii}$ & $\mathrm{iii}$ \\
\hline E & $\mathrm{i}$ & $\mathrm{ii}$ & $\mathrm{iii}$ \\
\hline
\end{tabular}

\section{LEARNING SPEED}

- Think about the time required to understand the lighting scenes from the selectors.

- Now rate each one of the selectors in terms of the time taken to understand the lighting scenes

\begin{tabular}{|c|c|c|c|}
\hline $\begin{array}{c}\text { Interface } \\
\text { Type }\end{array}$ & $\begin{array}{c}\text { Takes a lot } \\
\text { of time }\end{array}$ & $\begin{array}{c}\text { Takes some } \\
\text { time }\end{array}$ & $\begin{array}{c}\text { Takes very } \\
\text { little time }\end{array}$ \\
\hline A & i & ii & iii \\
\hline B & i & ii & iii \\
\hline C & i & ii & iii \\
\hline D & i & ii & iii \\
\hline E & i & ii & iii \\
\hline
\end{tabular}

\section{COMMENTS}


ADDITIONAL DESIGN SUGGESTIONS

If you have any new ideas for dimmers, colour controllers or scene selectors, please describe or draw in detail. 


\section{PERSONAL DATA}

To ensure that the survey has a good cross-section of people from all ages, gender and parts of the world, please provide some information about you.

AGE GROUP

\begin{tabular}{|c|c|c|c|c|}
\hline $16-24$ & $25-34$ & $35-44$ & $45-54$ & $\begin{array}{c}55 \& \\
\text { above }\end{array}$ \\
\hline
\end{tabular}

\section{Gender}

$$
\begin{array}{|l|l|}
\hline \text { Male } & \text { Female } \\
\hline
\end{array}
$$

\section{CONTINent}

\begin{tabular}{|c|c|c|c|c|c|}
\hline Africa & $\begin{array}{c}\text { America } \\
\text { (North) }\end{array}$ & $\begin{array}{c}\text { America } \\
\text { (South) }\end{array}$ & Asia & Europe & Oceania \\
\hline
\end{tabular}

THANK YOU VERY MUCH FOR YOUR PARTICIPATION! 


\section{APPENDIX 2}

\section{Questionnaire - Tangible interface study}




\section{EXPLORATION INTO LIGHTING CONTROL DEVICES}

Before you start the experiment, carefully observe the images of the two devices. If you were not informed that this is an experiment on lighting control, what would have been your general first impression of these devices? According to you, what would have been their possible use? Please describe.
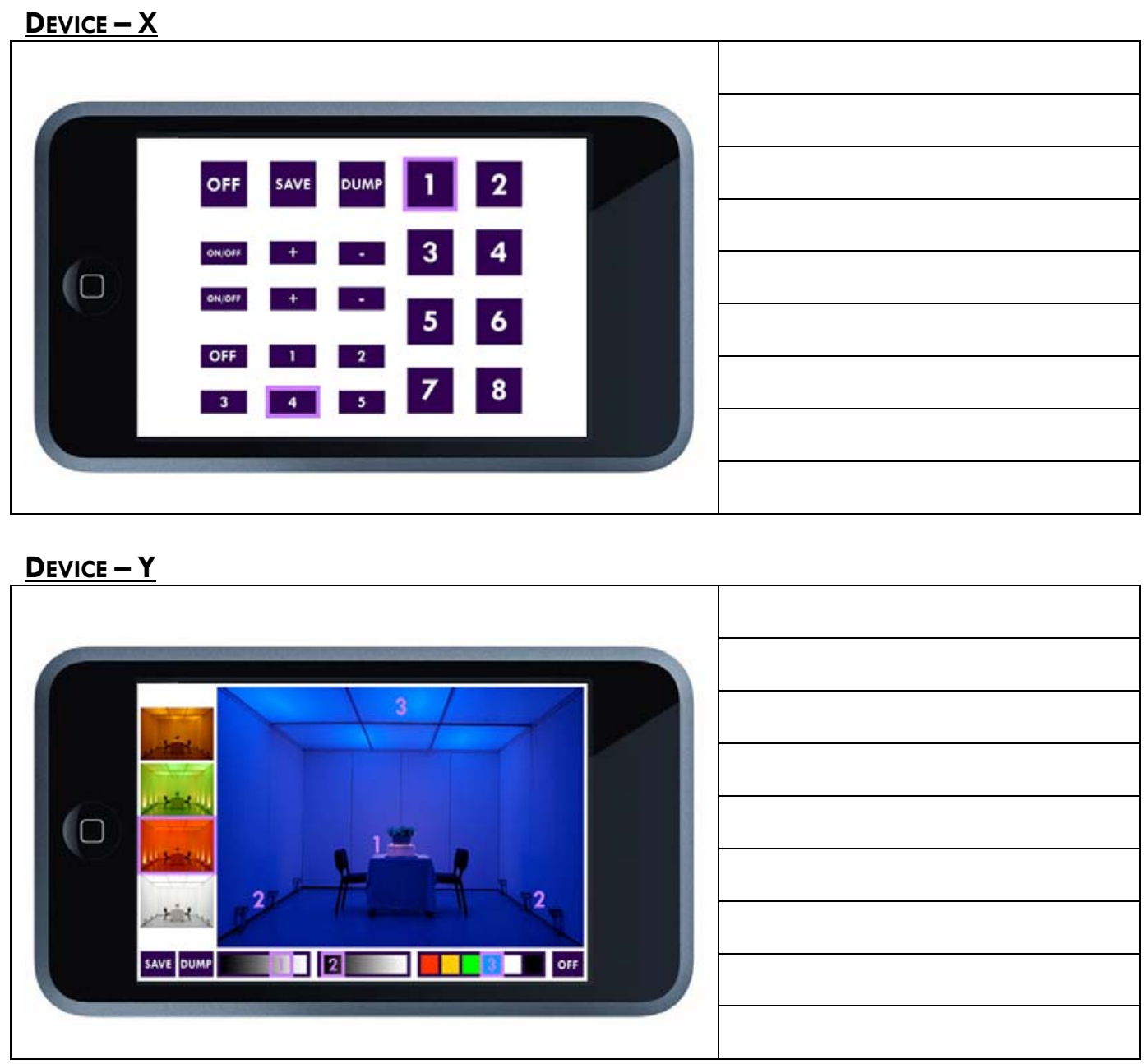

\section{QUESTIONNAIRE ANSWERING TIPS}

The next page describes the simple experiment using the lighting control devices that you have been asked to participate. Before you start using the devices, carefully read and follow the given instructions to familiarise with the device. After you have familiarised with the function and use of the device, please answer the questions in the following pages. Most of the questions are objective type questions with ranking scales, which requires you to tick the appropriate box. 


\section{ABOUT THE EXPERIMENT}

Take a careful look at the image projected on the screen (and below) and imagine yourself in this room. This experiment is meant to illuminate the room with different combinations of lights. The three types of lights available in this room are a luminous ceiling, a set of wall-washing lights and a central luminous object. The devices offer a range of options for controlling these lights to achieve different lighting scenarios of your choice. You can change the brightness and colours of these lights, as well as save and recall your favourite lighting scene.

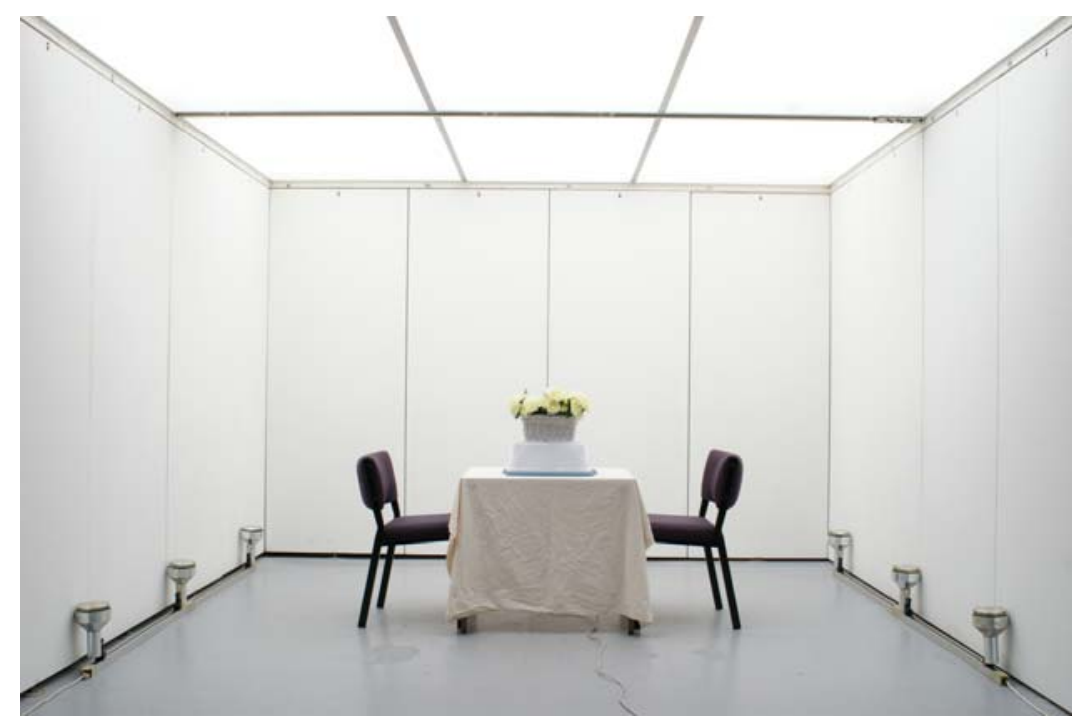

\section{INSTRUCTIONS}

- Try to identify visually (eyes only) first and then by using the control keys, which key is meant to change the brightness or colour of which light. Now gradually use the control keys to change the brightness or colour of these lights.

- Using the control keys, select a colour of light (e.g. red) from the ceiling, and different brightness levels (e.g. 50\% and $75 \%$ ) with the other two kinds of lighting. Now change the colours and brightness of the lights. Finally, try reverting to the same colour and brightness levels.

- Carefully observe the device while you change the brightness and colours of the lights.

- The keys on the device allow you to save your favourite combinations of colours and brightness as lighting scenes. Use the 'Save' key to save eight different combinations of colours and brightness of the lights as eight saved lighting scenes for future recall.

- The keys on the device allow you to recall your favourite lighting scene. After saving the eight different lighting scenes, try to recall your most favourite lighting scene from these saved options. 
- Now that you have performed the given functions with the first device, perform the same functions with second device. 


\section{LEARNING SPEED}

Activity: $\quad$ How long did it take to identify which key on the device is meant to change the brightness of the respective light?

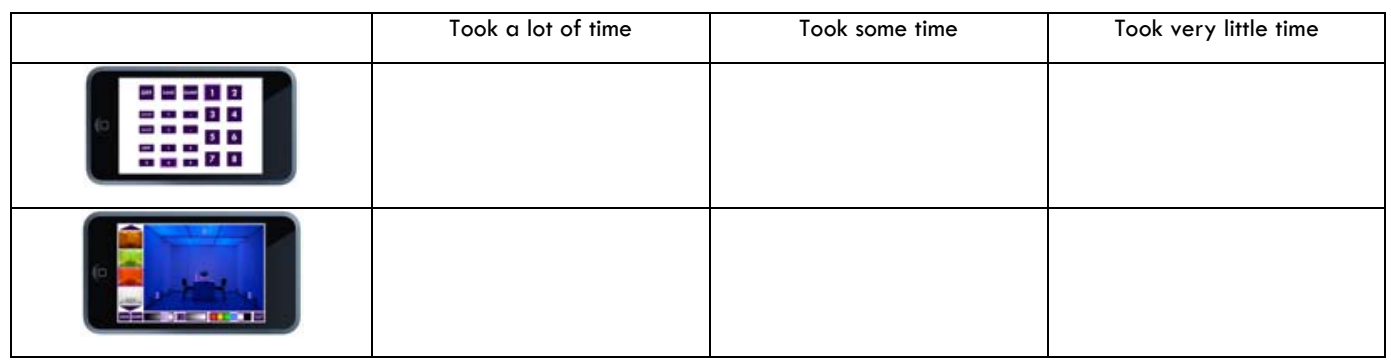

Activity: How long did it take to identify which key on the device is meant to change the colours of light?

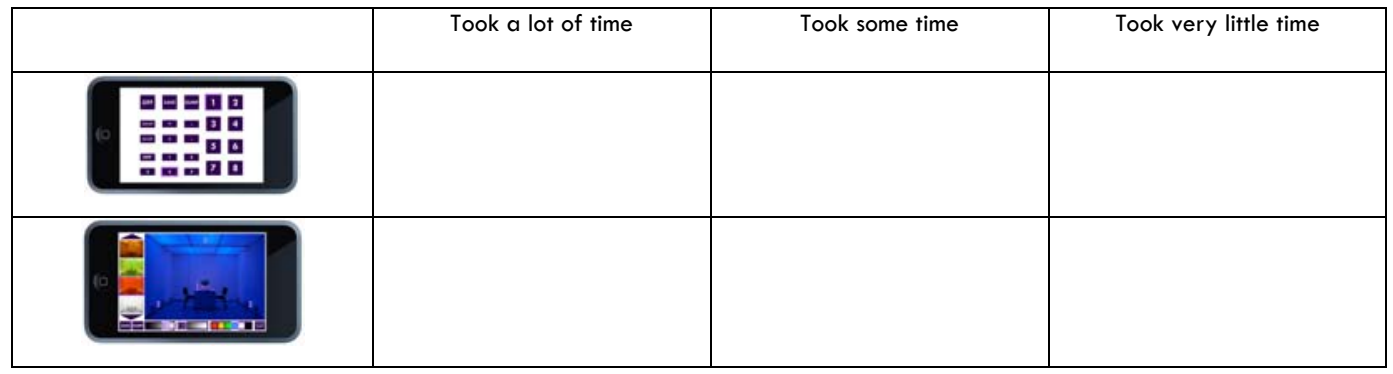

\section{EASE OF USE}

Activity: How easy was it to manipulate the brightness keys on the given device?

\begin{tabular}{|c|c|c|c|c|}
\hline & Very hard & Hard & Easy & Very easy \\
\hline 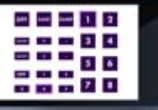 & & & & \\
\hline and & & & & \\
\hline
\end{tabular}

Activity: How easy was it to manipulate the colour keys on the given device?

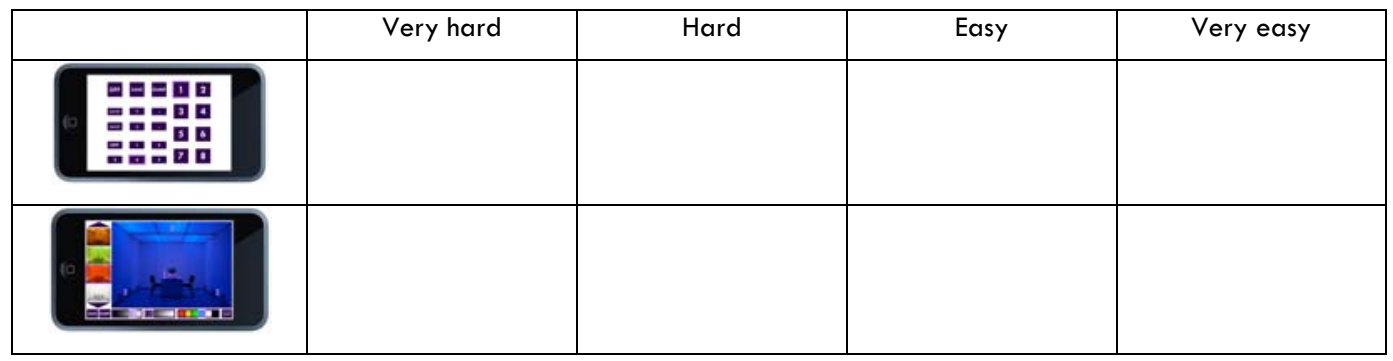




\section{ACCURACY}

Activity: How accurately were you able to repeat the selection of brightness with the given device?

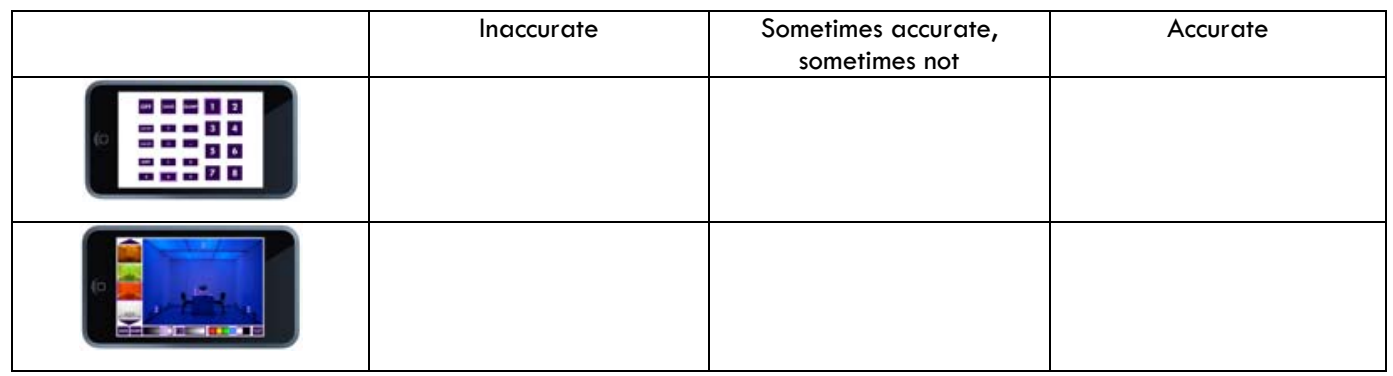

Activity: How accurately were you able to repeat the selection of colour with the given device?

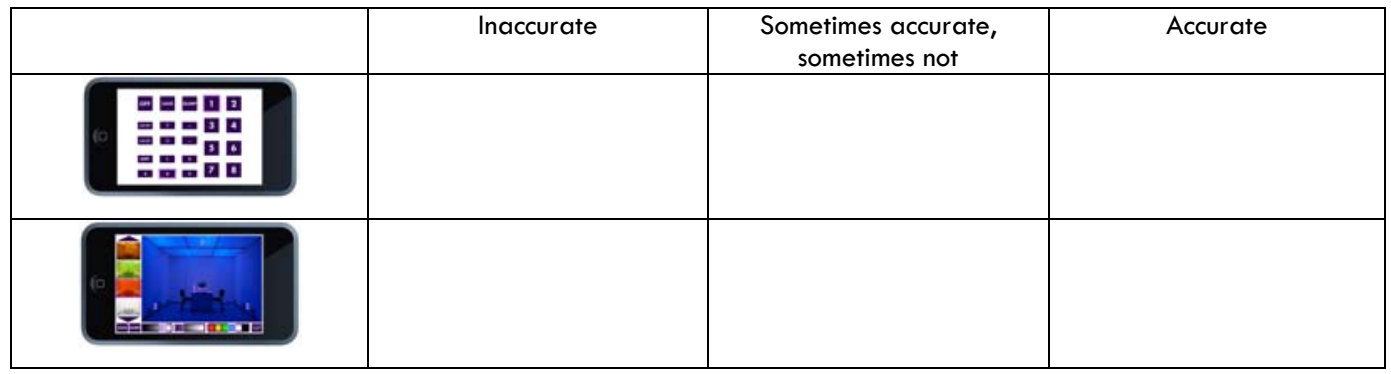

Activity: After how many attempts were you able to accurately recall your favourite lighting scene?

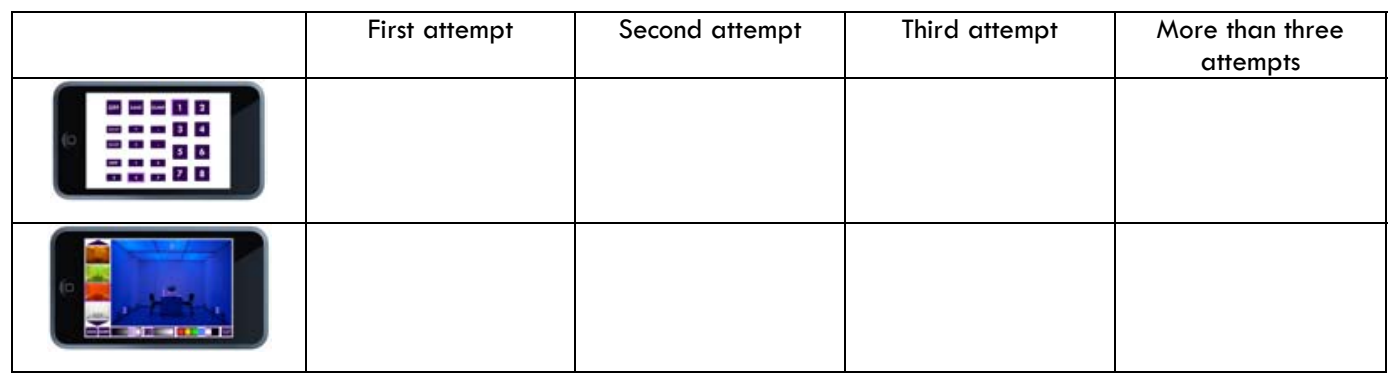

\section{RESPONSIVENESS}

Activity: How responsive is the device in giving feedback about the lighting in the room while you change the brightness and colours of the different lights?

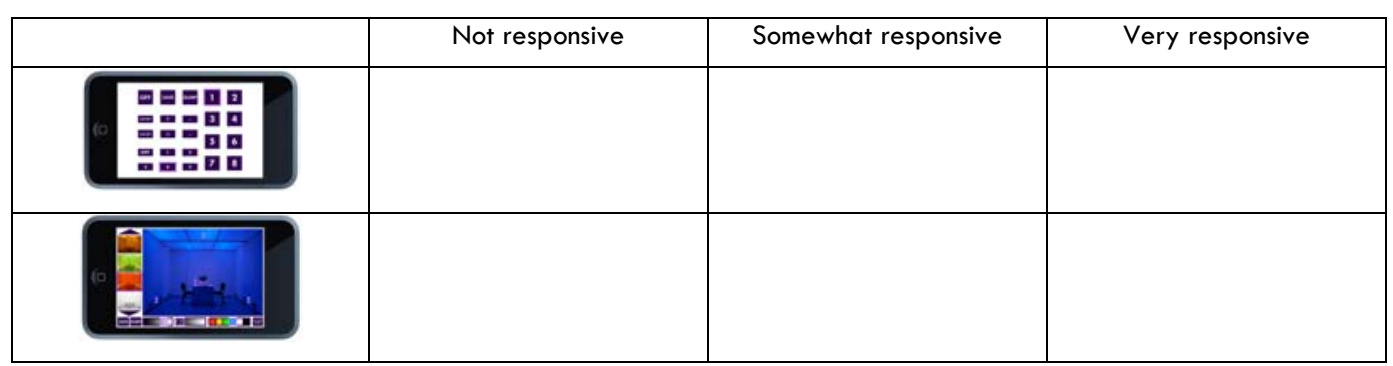




\section{COMMENTS}

Now that you have used both the devices, what is your general opinion about their designs? Can their designs be improved? Please describe.

\section{DEVICE - X}

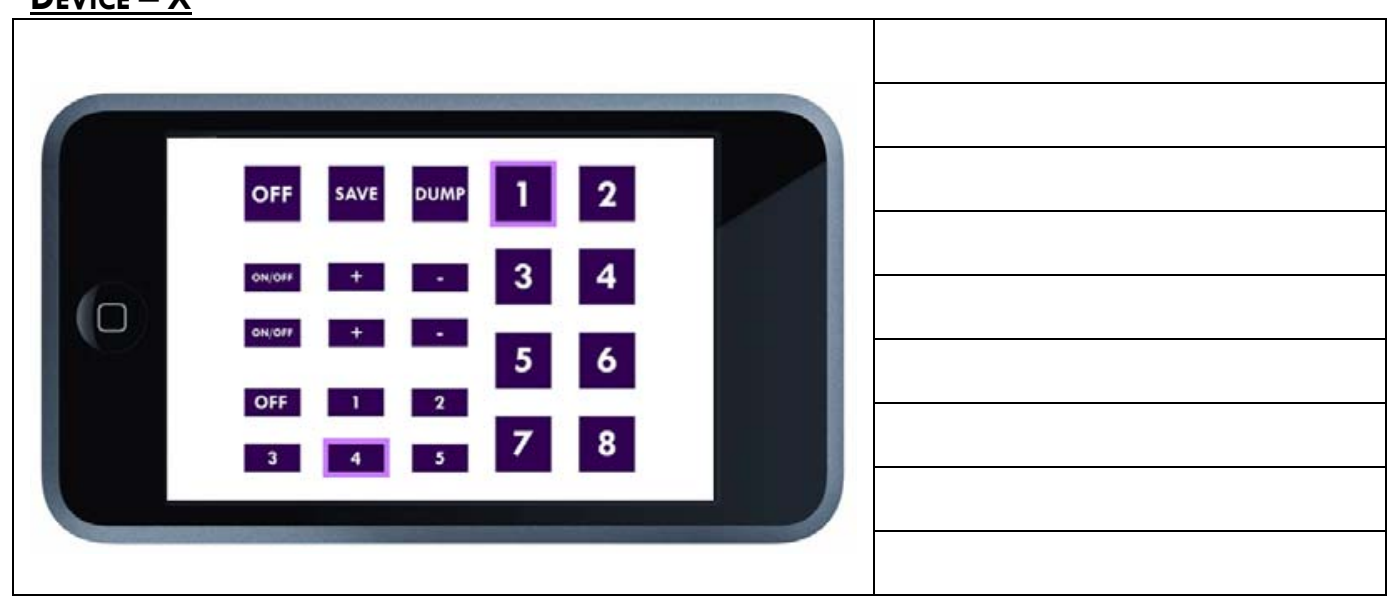

\section{DEVICE - $Y$}

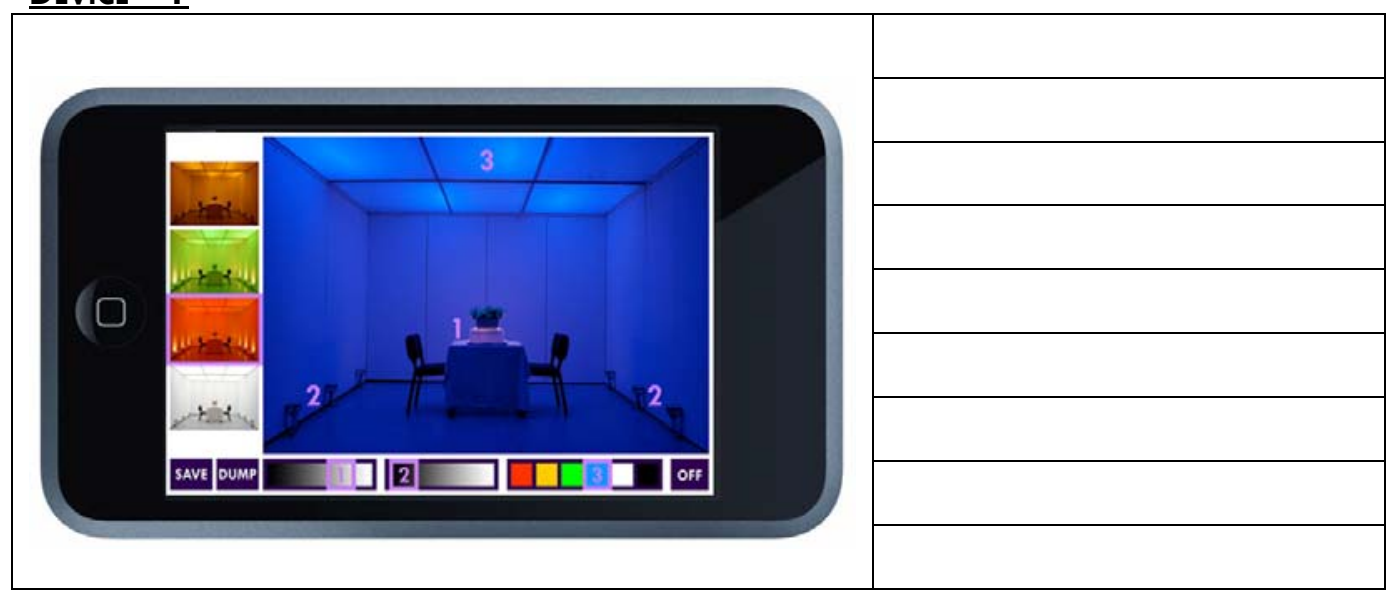

\section{PERSONAL DATA}

Finally, please provide some information about you!

AgE GROUP

\begin{tabular}{l|l|l|l|l}
$16-24$ & $25-34$ & $35-44$ & $45-54$ & $55 \&$ above
\end{tabular}

\section{GENDER}




\section{LIST OF CITED WORKS}

\section{References}

Allan, George (2003). A Critique of using Grounded Theory as a Research Method. Electronic Journal of Business Research Methods, $2(1), 1-10$.

Anderson, Craig A., Lindsay, James J., \& Bushman, Brad J. (1999). Research in the Psychological Laboratory: Truth or Triviality? Current Directions in Psychological Science, 8(1), 3-9.

Aston, J (2004). Comment on 'A study of opinion in offices with and without user controlled lighting' by T. Moore, D.J. Carter and A.I. Slater. [Comment on existing article]. Lighting Research \& Technology, 36(2), 144-145.

Atkinson, Richard C., \& Shiffrin, Richard M. (1968). Human Memory: A Proposed System and Its Control Process. In K. W. Spence \& J. T. Spence (Eds.), The Psychology of Learning and Motivation: Advances in Research and Theory (Vol. 2, pp. 89-195). New York, USA: Academic Press.

Baddeley, Alan D., \& Logie, Robert H. (1999). Working memory: The multiple component model. In Akira Miyake \& Priti Shah (Eds.), Models of Working Memory (pp. 28-61). New York, USA: Cambridge University Press. 
Balakrishnan, Vimala, \& Yeow, Paul H. P. (2010). Texting satisfaction: does age and gender make a difference. International Journal of Computer Science and Security, 1(1), 85-96.

Bischof, Markus, Conradi, Bettina, Lachenmaier, Peter, Linde, Kai, Meier, Max, Potzl, Philipp, et al. (2008, 18-20 February). Xenakis: combining tangible interaction with probability-based musical composition. Paper presented at the 2 nd international conference on Tangible and embedded interaction, Bonn, Germany.

Bongers, Bert (2002, 3 August). Interactivating Spaces. Paper presented at the 4th Annual Symposium on Systems Research in the Arts: Music, Environmental Design and The Choreography of Space, Baden-Baden, GERMANY.

Bordass, Bill, Leaman, Adrian, \& Bunn, Roderic (2007a). A guide for good design and implementation. In Building Controls Industry Association (Eds.), Controls for End Userspp. 26). Available from http://www.bcia.co.uk/documents/Controls $\% 20$ for $\% 20$ End $\% 20$ Us ers $\% 20$ guide.pdf

Bordass, Bill, Leaman, Adrian, \& Bunn, Roderic (2007b). Controls for End Users: a guide for good design and implementation. Reading, Berkshire, UK: Building Controls Industry Association (BCIA).

Brebner, J. M. T., \& Welford, A. T. (1980). Introduction: an historical background sketch. In A. T. Welford \& J. M. T. Brebner (Eds.), Reaction Times (pp. 1-23). New York, USA: Academic Press.

Brewer, Marilynn B. (2000). Research Design and Issues of Validity. In Harry T. Reis \& Charles M. Judd (Eds.), Handbook of Research 
Methods in Social and Personality Psychology (pp. 3-15). Cambridge, UK: Cambridge University Press.

Butler, Keith A. (1996). Usability engineering turns 10. Interactions, 3(1), 58-75.

Buur, Jacob, Jensen, Mads Vedel, \& Diajadiningrat, Tom (2004, 1-4 August ). Hands-only scenarios and video action walls: novel methods for tangible user interaction design. Paper presented at the 5th conference on Designing interactive systems: processes, practices, methods, and techniques, Cambridge, MA, USA.

Card, Stuart K., Moran, Thomas P., \& Newell, Allen (1983). The Psychology of Human Computer Interaction. London, UK: Lawrence Erlbaum Associates.

Carroll, Robert Todd (2003). The Skeptic's Dictionary: A Collection of Strage Beliefs, Amusing Deceptions, and Dangerous Delusions. Hoboken, NJ, USA: John Wiles \& Sons, Inc.

Cozby, Paul C. (2001). Methods in Behavioral Research (7 ed.). London, UK \& Toronto, CANADA: Mayfield Publishing Company.

Cyr, Dianne, Head, Milena, \& Ivanov, Alex (2005). Website Design and Mobility: Culture, Gender, and Age Comparisons. Paper presented at the Special Interest Group on Human-Computer Interaction 2005.

DiLouie, Craig. Lighting Controls Association: Education Express Retrieved 24 July 2008, from http://www.aboutlightingcontrols.org/index.shtml 
Djajadiningrat, Tom, Overbeeke, Kees, \& Wensveen, Stephan (2002). But how, Donald, tell us how? On the creation of meaning in interaction design through feedforward and inherent feedback. Paper presented at the International Conference on Designing Interactive Systems. Retrieved June 2007,

Djajadiningrat, Tom, Wensveen, Stephan, Frens, Joep, \& Overbeeke, Kees (2004). Tangible products: redressing the balance between appearance and action. Personal Ubiquitous Computing, 8(5), 294309.

Doll, William J., \& Torkzadeh, Gholamreza (1988). The measurement of end-user computing satisfaction. MIS Quarterly, $12(2), 259-274$.

Donders, F. C. (1969). On the speed of mental processes (W. G. Koster, Trans.). In H. C. J. Duijker, M. J. Langeveld, C. F. van Parreren \& O. Kilneberg (Eds.), Acta Psychologica (Vol. 30, pp. 412-431). Amsterdam, The NETHERLANDS: North Holland Publishing Company.

Dubuc, Bruno (2002, November 2007). The Brain from Top to Bottom Retrieved 08 January, 2009, from http://thebrain.mcgill.ca/flash/a/a $07 / a \quad 07 \mathrm{p} / \mathrm{a} 07 \mathrm{p}$ tra/a 07 p tra.html\#3

Dynalite (2008). An Introduction to the Dynalite Control System. In Dynalite (Ed.), Dynalite Intelligent Light. Mascot, NSW, AUSTRALIA: Dynalite Intelligent Light Pty. Ltd.

Egan, M. David, \& Olgyay, Victor W. (2002). Architectural Lighting (2 ed.). New York, USA: McGraw Hill. 
Escuyer, S., \& Fontoynont, M. (2001). Lighting controls: a field study of office workers' reactions. Lighting Research and Technology, 33(2), 77-94.

Fields, Bob, Wright, Peter C., \& Harrison, Michael D. (1997). Objectives, strategies and resources as design drivers. Paper presented at the IFIP TC13 International Conference on Human-Computer Interaction. from http://citeseer.ist.psu.edu/cache/papers/cs/1 336/http:zSzzSzww w.cs.york.ac.ukzSz bobzSz.zSzpaperszSzresourceinteract97.pdf/fields970bjectives.pdf

Gehnen, Markus (2008, December ). Tune the Light! ERCO Lichtbericht, 16-19.

Giles, David C. (2002). Advanced Research Methods in Psychology. London, UK and New York, USA: Routledge.

Glaser, Barney G., \& Strauss, Anselm L. (1967). Discovery of Grounded Theory: Strategies for Qualitative Research. Hawthorne, NY, USA: Aldine de Gruyter.

Hallnäs, Lars, \& Redström, Johan (2002). From use to presence: on the expressions and aesthetics of everyday computational things. ACM Transactions on Computer-Human Interaction, 9(2), 106-124.

Hayes, Andrew F., Gee, Travis L., Price, Ian R., Cooksey, Ray, \& Patrech, Adam (1998). Chapter 2: Research Design. Retrieved 15 June, 2009, from http://www.une.edu.au/WebStat/unit materials/index.htm 
Helvar (2007). User Interfaces. In Helvar (Ed.), Helvar - Lighting the Way Forward. Karkkila, FINLAND: Helvar.

Holmquist, Lars Erik, Schmidt, Albrecht, \& Ullmer, Brygg (2004). Tangible interfaces in perspective: Guest editors' introduction. Personal Ubiquitous Computing, 8(5), 291-293.

Hornecker, Eva (2006a). Physicality in Tangible Interaction: Bodies and the World. Paper presented at the 1 st International Workshop on Physicality. $\quad$ from http://www.physicality.org/physicality2006/Physicality2006Com plete.pdf\#page $=27$

Hornecker, Eva (2006b). Space and Place - setting the stage for social interaction. Interact Lab, Department of Informatics, University of Sussex.

Hornecker, Eva, \& Bruns, F. Wilhelm (2004). Interactive Installations Analysis - Interaction Design of a Sensory Garden Event. Paper presented at the IFAC/IFIP/IFORS/IEA Symposium on the Analysis, Design and Evaluation of Human-Machine Systems. from http://www.ehornecker.de/Papers/HorneckerBrunslFAC.pdf

Hornecker, Eva, \& Buur, Jacob (2006). Getting a grip on tangible interaction: a framework on physical space and social interaction. Paper presented at the SIGCHI Conference on Human Factors in Computing Systems, Montreal, Quebec, CANADA.

Howlett, Owen (2001). Comment on 'Lighting controls: a field study of office workers' reactions' by $S$ Escuyer and $M$ Fontoynont. [Comment on existing article]. Lighting Research \& Technology, $33(2), 94$. 
Huitt, W (1998). Critical thinking: An overview. Retrieved 30 May 2009, from Valdosta State University: http://chiron.valdosta.edu/whuitt/col/cogsys/critthnk.html

Jennings, Judith D., Rubinstein, Francis M., DiBartolomeo, Dennis, \& Blanc, Steven L. (2000). Comparison of Control Options in Private Offices in an Advanced Lighting Controls Testbed. Journal of the IESNA.

Jensen, Mads Vedel, Buur, Jacob, \& Diajadiningrat, Tom (2005, August 20 - 24). Designing the user actions in tangible interaction. Paper presented at the 4th Decennial Conference on Critical Computing: Between Sense and Sensibility, Aarhus, DENMARK.

Kaufman, Alan S. (2003). Practice Effects. The Clinical Cafe. Retrieved 21 June, 2009, from http://www.speechandlanguage.com/cafe/13.asp

Keyson, David V. (1997). Touch in user interface navigation. Paper presented at the IEE Colloquium on Developments in Tactile Displays. from http://ieeexplore.ieee.org/iel4/5577/14913/00676703.pdf?tp $=\&$ isnumber $=$ \&arnumber $=676703$

Kinoshita, Gen-Ichiro, Aida, Shuhei, \& Mori, Masahiro (1975). A pattern classification by dynamic tactile sense information processing. Pattern Recognition, 7(4), 243-251.

Kosinski, Robert J. (September 2008). A Literature Review on Reaction Time Retrieved 08 January, 2009, from http://biae.clemson.edu/bpc/bp/Lab/1 10/reaction.htm\#Type\%2 Oof\%20Stimulus 
Krippendorff, Klaus, \& Butter, Reinhart (1984). Product Semantics: Exploring the symbolic qualities of form. Innovation: The journal of the Industrial Designers Society of America, 3(2), 4-9.

Krippendorff, Klaus, \& Butter, Reinhart (2008). Semantics: Meanings and contexts of artifacts. In Hendrik N. J. Schifferstein \& Paul Hekkert (Eds.), Product Experience (pp. 353-376). San Diego: Elsevier.

Lam, William M.C. (1977). Perception and Lighting as Formgivers for Architecture. New York, USA: McGraw Hill, Inc.

Lane, David M. (14 June 2009). Carryover effects. HyperStat Online Retrieved 21 June, 2009, from http://davidmlane.com/hyperstat/A134100.html

Leaman, Adrian (1995). Dissatisfaction and Office Productivity. Facilities, $13(2), 13-19$.

Levine, Gustav, \& Parkinson, Stanley (1994). Experimental Methods in Psychology. Hillsdale, NJ, USA: Lawrence Erlbaum Associates.

Lincoln, Yvonna S., \& Guba, Egon G. (1986). But is it rigorous? Trustworthiness and authenticity in naturalistic evaluation. In D.D. Williams (Ed.), Naturalistic evaluation (pp. 73-84). San Francisco, CA, USA: Jossey-Bass.

Luce, R. Duncan (1986). Response times: Their Role in Inferring Elementary Mental Organization. New York, USA: Oxford University Press.

Maeda, John (2006). The Laws of Simplicity. Cambridge, Massachusetts, USA \& London, UK: The MIT Press. 
Maniccia, Dorene, Rutledge, Burr, Rea, Mark S., \& Morrow, Wayne (1999). Occupant Use of Manual Lighting Controls in Private Offices. Journal of the IESNA, 28(2), 42-56.

Mayes, J. T., \& Fowler, C. J. (1999). Learning technology and usability: a framework for understanding courseware. Interacting with Computers, $11(5), 485-497$.

Messick, Samuel (1995). Validity of Psychological Assesment: Validation of Inferences From Person's Responses and Performance as Scientific Inquiry Into Score Meaning. Americal Psychologist, 50(9), $741-749$.

Miller, Herman (2001). Lighting in the Workplace Retrieved September 2007, from http://www.hermanmiller.com.mx/pdf/wp lighting in wkpl.pdf

Miller, Jeff, \& Low, Kathy (2001). Motor processes in simple, go/no-go, and choice reaction time tasks: A psychophysiological analysis. Journal of Experimental Psychology: Human Perception and Performance, 27(2), 266-289.

Minke, Amy (1997). Conducting Repeated Measures Analyses: Experimental Design Considerations. Retrieved 15 June, 2009, from http://ericae.net/ft/tamu/Rm.htm

Montenegro, Xenia (2004). Non-Response to Private and Sensitive Survey Questions. Paper presented at the Annual meeting of the American Association for Public Opinion Research. Retrieved 15 December 2008, from http://www.allacademic.com/meta/pl 15864 index.html 
Moore, Thomas, Carter, David J., \& Slater, Anthony I. (2002a). A field study of occupant controlled lighting in offices. Lighting Research and Technology, 34(3), 191-202.

Moore, Thomas, Carter, David J., \& Slater, Anthony I. (2002b). User attitudes toward occupant controlled office lighting. Lighting Research and Technology, 34(3), 207-216.

Moore, Thomas, Carter, David J., \& Slater, Anthony I. (2003). Long-term patterns of use of occupant controlled office lighting. Lighting Research and Technology, 35(1), 43-57.

Moore, Thomas, Carter, David J., \& Slater, Anthony I. (2004). A study of opinion in offices with and without user controlled lighting. Lighting Research and Technology, 36(2), 131-144.

Muller, Michael J., Matheson, Lisa, Page, Colleen, \& Gallup, Robert (1998). Methods \& tools: participatory heuristic evaluation. interactions, 5(5), 13-18.

Muller, Michael J., Wildman, Daniel M., \& White, Ellen A. (1993). 'Equal opportunity' PD using PICTIVE. Communications of the ACM, 36(6), 64-66.

Nielsen, Jakob, \& Mack, Robert L. (Eds.). (c1994). Usability inspection methods. New York, USA: Wiley.

Norman, Donald A. (1990 c1988). The Design of Everyday Things (1 ed.). New York, USA: Doubleday/Currency.

Norman, Donald A., \& Shallice, Tim (2000). Attention to action: Willed and automatic control of behaviour. In Michael S. Gazzaniga (Ed.), 
Cognitive Neuroscience: a reader (pp. 376-390). London, UK: Blackwell Publishers.

Overbeeke, Kees, Diajadiningrat, Tom, Hummels, Caroline, \& Wensveen, Stephan (2002). Beauty in usability. In WS Green \& PW Jordan (Eds.), Pleasure with products: Beyond usability (pp. 9-18). London, UK: Taylor \& Francis.

Palmer, Stephen E. (1999). Vision Science: Photons to Phenomenology. Cambridge, Massachusetts, USA: MIT Press.

Preece, Jenny, Rogers, Yvonne, \& Sharp, Helen (2002). Interaction Design - beyond human-computer interaction ( 1 ed.). New York, USA: John Wiley \& Sons, Inc.

Ramstein, Christophe, Martial, Odile, Dufresne, Aude, Carignan, Michel, Chass, Patrick, \& Mabilleau, Philippe (1996). Touching and hearing GUI's: design issues for the PC-Access system. Paper presented at the 2nd Annual ACM SIGACCESS Conference on Assistive Technologies. from http://delivery.acm.org/10.1145/230000/228349/p2ramstein.pdf?key $1=228349 \&$ key $2=1651983021 \&$ coll=GUIDE\&d $\underline{I=\text { GUIDE } \& \text { CFID }=56414362 \& \text { CFTOKEN }=34477530}$

Rea, Mark S. (Ed.). (2000). The IESNA lighting handbook : reference \& application. New York, USA: Illuminating Engineering Society of North America.

Robson, Colin (2002). Real World Research (2 ed.). Oxford, UK: Blackwell Publishing. 
Ross, Philip, \& Keyson, David V. (2007). The case of sculpting atmospheres: towards design principles for expressive tangible interaction in control of ambient systems. Personal Ubiquitous Computing, $11(2)$, 69-79.

Rubidge, Sarah, \& MacDonald, Alistair (2004). Sensuous Geographies: a multi-user interactive/responsive installation. Digital Creativity, $15(4), 245-252$.

Rubinstein, Richard, \& Hersh, Harry (1984). The Human Factor: Designing Computer Systems for People. Bedford, Massachusetts, USA: Digital Press.

Salkind, Neil J. (2007). Statistics for People Who (Think They) Hate Statistics (Excel ed.). Thousand Oaks, CA, USA: Sage Publications Inc.

Scaife, Michael, Rogers, Yvonne, Aldrich, Frances, \& Davies, Matt (1997). Designing for or designing with? Informant design for interactive learning environments. Paper presented at the SIGCHI Conference on Human Factors in Computing Systems. from http://delivery.acm.org/10.1 145/260000/258789/p343-

scaife.pdf?key $1=258789 \&$ key $2=8553043021 \&$ coll $=$ GUIDE $\& d I=$ GUIDE\&CFID $=16880613 \&$ CFTOKEN $=63489351$

Schmorrow, Dylan, Kay M. Stanney, Glenn Wilson, and Peter Young (2006). Augmented Cognition in Human-System Interaction. In Gavriel Salvendy (Ed.), Handbook of Human Factors and Ergonomics (pp. 1364-1383). New Jersey, USA: John Wiley \& Sons. 
Schuler, Douglas, \& Namioka, Aki (Eds.). (1993). Participatory Design: Principles and Practices (Illustrated ed.). Mawah, NJ, USA: Lawrence Erlbaum Associates.

Sekaran, Uma (2000). Research Methods for Business (3 ed.). New York, NY, USA: John Wiley \& Sons, Inc.

Sharma, Rajeev, Pavlovic, Vladimir I., \& Huang, Thomas S. (1998). Toward multimodal human-computer interface. Paper presented at the IEEE. from http://ieeexplore.ieee.org/iel3/5/14574/00664275.pdf?tp=\&is number $=\&$ arnumber $=664275$

Simon, Herbert A. (1957). Models of Man: Social and Rational. New York, USA: John Wiley \& Sons.

Simpson, Robert S. (2003). Lighting Control - Technology and Applications. Burlington, USA: Focal Press.

Sohlberg, McKay Moore, \& Mateer, Catherine A. (1989). Cognitive Rehabilitation: An Integrative Neuropsychological Approach. New York, USA: Guilford Press.

Steffy, Gary (2001). Architectural Lighting Design (2 ed.). New York, USA: John Wiley \& Sons Ltd.

Tegin, Johan, \& Wikander, Jan (2005). Tactile sensing in intelligent robotic manipulation - a review. Industrial Robot: An International Journal, 32(1), 64-70.

Thomassen, Aukje (2003). In Control: Engendering a continuum of flow of a cyclic process within the context of potentially disruptive GUI 
interactions. Unpublished PhD Thesis, Hogeschool Voor de Kunsten Utrecht, THE NETHERLANDS.

Tidwell, Jenifer (2005). Designing Interfaces (1 st ed.). Sebastopol, CA, USA: O'Reilly Media, Inc.

Ullmer, Brygg, \& Ishii, Hiroshi (2001). Emerging Frameworks for Tangible User Interfaces. In John M. Carroll (Ed.), Human-Computer Interaction in the New Millenium (pp. 579-601). Boston, USA: Addison-Wesley.

Underkoffler, John, \& Ishii, Hiroshi (1999, 15-20 May). Urp: a luminoustangible workbench for urban planning and design. Paper presented at the SIGCHI conference on Human factors in computing systems: the $\mathrm{CHI}$ is the limit, Pittsburgh, Pennsylvania, USA.

Weiser, Mark (1999). The computer for the 21 st century. SIGMOBILE Mobile Computing and Communications Review, 3(3), 3-11.

Welford, A.T (1980). Choice reaction time: Basic concepts. In A. T. Welford \& J. M. T. Brebner (Eds.), Reaction Times (pp. 73-128). New York, USA: Academic Press.

Wendorf, Craig A. (1997, 12 March 2004). Part V: Analysis of Variance (ANOVA). Manuals for Univariate and Multivariate Statistics. Retrieved 17 March, 2009, from http://www.uwsp.edu/psych/cw/statmanual/index.html

Winchip, Susan M (2005). Designing a Quality Lighting Environment. New York, USA: Fairchild Publications. 
Wojciechowski, Adam, \& Zakrzewska, Danuta (2006). The Influence of Cultural Preferences on User Interface Design - Polish Case Study Advances in Computer, Information, and Systems Sciences, and Engineering (pp. 465-472): Springer Netherlands. 